\title{
QoE Awareness For the Mobile Termination Rates Monopoly LIBERATION
}

Dissertation Submitted to the Faculty of Business, ECONOMICS AND INFORMATICS OF THE UNIVERSITY OF ZURICH

To Obtain the Degree of DOKTOR Der Wissenschaften, DR. SC. (Corresponds to Doctor of Science, PhD)

\author{
Presented by \\ Christos Tsiaras \\ FROM GREECE
}

ApProved IN JULY 2016

AT THE REQUEST OF

Prof. Dr. Burkhard Stiller

Prof. Dr. Peter Reichl 


\section{University of}

Zurich $^{\mathrm{UzH}}$

The Faculty of Business, Economics and Informatics of the University of Zurich hereby authorizes the printing of this dissertation, without indicating an opinion of the views expressed in the work.

Zurich, July 20, 2016

Chairwoman of the Doctoral Board: Prof. Dr. Elaine M. Huang 


\section{Abstract}

IN mobile communication the mobile termination service involves charges between Mobile (Virtual) Network Operators (MvNOs). Furthermore, only the MvNO of the callee is able to terminate his calls. Thus, in the MvNOs call-termination market there is only one player profiting from Mobile Termination Rates (MTR); in turn this market is considered by regulators to be a "de-facto" monopoly, since the early days of the introduction of commercial mobile communication services. Given this monopoly fact, the only solution against a potential speculation by MvNOs was the regulation of the MTR.

In a monopoly there is a corporation that is the only seller of a good or a service, and thus it can define the price. However, monopolies can be divided into two categories, the naturally defined and the market-defined monopolies. The power market in many countries is considered to be a natural monopoly, and the main reason is that there is usually only one power-wire reaching each house. Thus, only the company that owns the delivery network can provide power services. The termination service in mobile communication until the $4 \mathrm{G}$ is also considered to be a monopoly. However, this is a market-defined monopoly, since there is no physical limitation (e.g., wires) for reaching a mobile user.

The $2.5 \mathrm{G}$ and higher mobile communication technology allows for flexible charging mechanisms, such as on-demand MvNO selection combined with non-static MTR, that could overcome the Mobile Termination Rates monopoly obstacle. Thus, this thesis shows that the mobile termination service is not a "de-facto" monopoly since $2.5 \mathrm{G}$. To show that, the Auctionbased Charging User-centric System "AbaCUS" is proposed in this thesis as a $2.5 \mathrm{G}$ and higher overlay, where MvNOs will participate in an auction to allow competition that aims to increase end-users Quality-of-Experience (QoE). MvNOs will charge for QoE while bidding on economic variables, such as MTR per Quality-of-Service ( $\mathrm{OoS}$ ) variables, such as the sound quality during a call and network-access guarantees, in a manner that the 
MvNO which will increase end-users QoE will be the one selected by the Auction Authority $\left(\mathrm{Au}^{2}\right)$ to provide the termination service.

This thesis is arguing that charging for QoE is the key to overcome the monopoly of the MTR market. An Axiomatic QoE model (AQX), which considers simultaneously technical and non-technical parameters, is designed in this thesis, to estimate end-user's QoE for a certain service (e.g., the mobile termination service). Such service can be provided by competing MvNOs that can use $\mathrm{QoE}$ as the AbaCUS auction bidding metric defining which $\mathrm{MvNO}$ will provide the termination service. AQX is a generic, since it is not service-specific, QoE model that can be applied in multiple domains. Thus, the roadmap of selecting all AQX parameters needed is presented for the well-studied Voice over Internet Protocol (VoIP) scenario. This scenario is selected as a reference to present and evaluate AQX, since there are several existing QoE models. AQX has been proven to outperform the state-of-the-art QoE estimation models for VoIP, IQX Hypothesis and the ITU-T E-Model, in several cases.

This thesis proposes and implements prototypically the technical mechanism needed to break the mobile termination service monopoly, an automatic and on-demand MvNO selection mechanism, and evaluates this mechanism in terms of time and energy efficiency, showing that the proposed solution is technically feasible. Finally, this thesis presents economic and technical incentives for the mobile communications stakeholders (MvNOs, end-users, and regulators), to adopt the idea, and implement the technical mechanism proposed by AbaCUS, to facilitate a competitive termination-service environment in the mobile call-termination services market. 


\section{Kurzfassung}

IN der Mobilkommunikation beinhalten Mobilterminierungsraten (MTR) die Gebühren zwischen (virtuellen) Mobilfunkanbietern (MvNOs). Nur der MvNO des Angerufenen ist in der Lage, dessen Anrufe zu terminieren. Somit profitiert im Mobilterminierungsmarkt lediglich ein MvNO von den MTR. Dementsprechend wird dieser Markt von Aufsichtsbehörden bereits seit der Entstehung kommerzieller Mobilkommunikationsdienste als "de-facto"-Monopol angesehen. Die Regulation der MTR war die einzige Möglichkeit potentiellen Spekulationen der MvNOs vorzubeugen.

Ein Angebotsmonopol ist dadurch gekennzeichnet, dass es ausschliesslich einen Anbieter einer Ware oder einer Dienstleistung gibt und dieser daher den Preis bestimmen kann. Monopole können als natürlich und vom Markt definiert kategorisiert werden. Der Energiemarkt ist in vielen Ländern als natürliches Monopol anzusehen, da beispielsweise ein Haus für gewöhnlich nur über eine Stromleitung an das Stromnetz angebunden ist. Dementsprechend ist ausschliesslich der Anbieter, dem das Stromnetz gehört, in der Lage die Verbraucher mit Strom zu versorgen. Der Mobilterminierungsmarkt bis $4 \mathrm{G}$ wird auch als Monopol angesehen. Hierbei handelt es sich allerdings um ein vom Markt definiertes Monopol, da es keine physische Limitierung (wie beispielsweise Kabel) gibt, um den Mobilfunknutzer zu erreichen.

Mobilfunktechnologien ab 2.5 G ermöglichen flexible Bepreisungsmechanismen, wie beispielsweise die optionale Wahl des MvNO gekoppelt mit nicht-statischen MTR, mit denen sich das Monopol beim Mobilterminierungsmarkt für Sprachanrufe überwinden liesse. Dementsprechend zeigt diese Dissertation, dass der Mobilterminierungsmarkt seit 2.5 G kein "de-facto"-Monopol mehr ist. Um dies zu belegen, wird das Auctionbased Charging User-centric System "AbaCUS” entwickelt. Bei diesem handelt es sich um ein Overlay für ${ }^{2.5} \mathrm{G}$ und neuere Mobilfunktechnologien, welches das Konkurrieren von MvNOs durch die Teilnahme an Auktionen, die darauf abzielen die Quality-of-Experience (QoE) der 
Endnutzer zu erhöhen, erlaubt. Die Gebote in diesen Auktionen sind die QoE, also ökonomische Variablen (wie beispielsweise MTR), die die MvNOs in Abhängigkeit von der QoS (wie beispielsweise Tonqualität und Verbindungswahrscheinlichkeit) verlangen. Der MvNO, der die Auktion gewinnt und somit den Anruf des Endnutzers terminiert, wird von einer Auction Authority $\left(\mathrm{Au}^{2}\right)$ zu Gunsten der QoE des Endnutzers ausgewählt.

Diese Dissertation zeigt auf, dass das Konkurrieren von MvNOs bezüglich der QoE der Schlüssel ist, um das Monopol des Mobilterminierungsmarktes zu brechen. Hierfür wird ein axiomatisches QoEModell (AQX), das simultan technische und nicht-technische Parameter berücksichtigt, entworfen, um die QoE des Endnutzers für einen bestimmten Dienst zu schätzen (beispielsweise das Terminieren mobiler Anrufe). Solch ein Dienst kann von konkurrierenden MvNOs geliefert werden, welche die QoE als AbaCUS-Auktionsgebotmetrik nutzen, um zu bestimmen, welcher MvNO den Anruf terminiert. AQX ist ein QoEModell, das generisch ist, da es nicht dienstspezifisch ist, und somit in zahlreichen Domänen angewendet werden kann. Daher wird das Vorgehen um alle benötigten AQX-Parameter zu selektieren, für das gut untersuchte Voice over Internet Protocol (VoIP)-Szenario präsentiert. Dieses Szenario wird als Referenz gewählt, um AQX zu präsentieren und zu evaluieren, da für dieses Scenario zahlreiche QoE-Modelle existieren. Die Überlegenheit von AQX über die aktuellen QoE-Modelle für VoIP, das IQXHypothesenmodell und das ITU-T E-Modell, wird für zahlreiche Fälle demonstriert.

Diese Dissertation entwickelt und implementiert prototypisch den technischen Mechanismus, der benötigt wird, um das Angebotsmonopol im Mobilterminierungsmarkt durch einen automatischen, optionalen MvNO-Selektionsmechanismus zu brechen. Dieser Mechanismus wird hinsichtlich Zeit- und Energieeffizienz evaluiert, um zu zeigen, dass die vorgeschlagene Lösung technisch umsetzbar ist. Abschliessend präsentiert diese Dissertation ökonomische und technische Anreize für alle betroffenen Parteien (MvNOs, Endverbraucher und Aufsichtsbehörden) den vorgeschlagenen Selektionsmechanismus anzunehmen und durch AbaCUS zu implementieren und somit einen kompetitiven Mobilterminierungsmarkt zu schaffen. 


\section{Contents}

ABStract

KuRZFASSUNG

iii

1 INTRODUCTION 1

1.1 QoE ...................... 5

1.2 Mobile Termination Rates Monopoly Liberation . . . . . 6

1.3 On-demand MvNO Selection ........... 7

1.4 Thesis Contributions ............... 7

1.5 Thesis Outline ............... 8

2 RELATED WORK 11

2.1 Mobile Voice Services ............... 11

2.1.1 MTR Monopoly .............. 13

2.2 Auction Mechanisms ............... 15

2.3 Flexible MvNO Selection . . . . . . . . . . . . 17

2.3.1 Apple Patent ............... 18

2.3.2 Apple SIM Card .............. 18

2.3.3 Flexible MNO Selection Demands . . . . . . 19

2.4 QoE ....................... 19

2.4.1 QoE Models ............... 20

2.4.2 Charging for $\mathrm{QoE}$............ 24

2.5 Contribution Opportunities ............ 26

3 AQX: Axiomatic QoE Model 29

3.1 QoE Formalization in AQX . . . . . . . . . . 30

3.2 Single-variable QoE Functions ........... . 35

3.2.1 IV QoE Function ............. 35

3.2.2 AV QoE Function ............. 36

3.3 Influence Factors . . . . . . . . . . . . . 38

3.3.1 IV Influence Factors Calculation . . . . . . . 39

3.3.2 AV Influence Factors Calcultation . . . . . . . 42 
3.4 Multi-variable QoE Function . . . . . . . . . . . 44

3.5 ITU-T MOS-Compliant QoE Functions . . . . . . . . 46

3.6 Concurrent Economic and Technical Parameters Consideration ..................... 48

3.7 Chapter Summary . . . . . . . . . . . . . . . 49

4 AQX IN VoIP 51

$4.1 \quad$ Experimental Setup . . . . . . . . . . . . 53

4.1.1 Hardware and Software Architecture ..... . 53

4.1.2 Experimental Procedure .......... 54

4.2 AQX Parameters in VoIP . . . . . . . . . . . 55

4.2.1 Single Variables ............. 57

4.2.2 Multiple Variables Combination . . . . . . . . 63

4.3 Chapter Summary . . . . . . . . . . . 65

5 The Design to Overcome the MTR Monopoly 67

5.1 Stakeholders Analysis . . . . . . . . . . . . 70

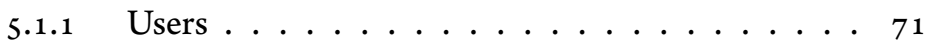

5.1 .2 Policy Makers ... . . . . . . . . 74

5.1.3 Connectivity and Information Providers . . . . 75

5.1.4 Technology Makers and Infrastructure Providers . 76

5.2 QoE of a Mobile Call According to AQX . . . . . . . 77

5.2.1 QoE-related Variables Identification . . . . . . . . . 77

5.2.2 QoE Variables IV or AV Classification . . . . . . . 78

5.2.3 Minimum and Maximum QoE Variable Values Identification . . . . . . . . . . . 78

5.2.4 Desired $\left(x_{\circ}\right)$ QoE Variable Values Identification . 79

5.2.5 Influence Factors $(m)$ Identification . . . . . . . 80

5.2.6 Importance Factors $(w)$ Identification . . . . . . 81

5.2.7 Generic QoE of a Mobile Call . . . . . . . . . 81

5.3 QoS Class $($ QoS-C) . . . . . . . . . . 86

5.4 TeR Class $($ TeR-C) . . . . . . . . . . . . 88

5.5 AbaCUS: Auction-based Charging User-centric System . . 89 5.5.1 AbaCUS Bidding Metric, Auction, and $\mathrm{Au}^{2} \ldots .91$

5.6 AbaCUS Assumptions . . . . . . . . . . . . 93

5.7 Chapter Summary . . . . . . . . . . . . . . 93 
6 Automatic and On-demand MNO Selection Mechanism

6.1 AT Commands Towards the Automatic and On-demand

MNO Selection . . . . . . . . . . . . . . . 97

6.2 MNO Selection Mechanism in Android . . . . . . . . . 99

6.2.1 Obtaining the Original Android Framework . . . 101

6.2.2 Creating a Customized Android Framework . . . 101

6.2.3 Modifying the Eclipse Access Rule . . . . . . . 102

6.2.4 Invoking the MNO Selection Mechanism . . . . . 103

6.3 Evaluation of the MNO Selection Mechanism . . . . . . . 104

6.3.1 Time Consumption between MNO Switching . . 105

6.3.2 MNO Switching Energy Consumption . . . . . 109

6.4 AbaCUS E2E Calling-time . . . . . . . . . . . 113

6.5 Chapter Summary . . . . . . . . . . . . . 114

7 Summary, Conclusions, and Future Work 117

7.1 Overcoming the MTR Monopoly . . . . . . . . 118

7.1.1 Automatic and On-demand MNO Selection . . . 119

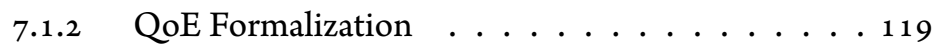

7.2 Conclusions ................... 120

$7 \cdot 3$ Future Work .................. 121

$\begin{array}{lr}\text { REFERENCES } & 123\end{array}$

$\begin{array}{lr}\text { Other Author Publications } & 135\end{array}$

$\begin{array}{ll}\text { APPENDIX } & 137\end{array}$

A.1 Formal Proofs of AQX Equations . . . . . . . . 137

$\begin{array}{lr}\text { List OF Figures } & 142\end{array}$

$\begin{array}{lr}\text { List Of TABles } & 144\end{array}$

$\begin{array}{lr}\text { ACKNOWLedgments } & 145\end{array}$

$\begin{array}{ll}\text { Curriculum Vitae } & 147\end{array}$

$\begin{array}{lr}\text { GlOSSARY } & 149\end{array}$ 



\section{1 \\ Introduction}

$\prod$ of Mobile (Virtual) Network Operators (MvNOs) end-users, while adjusting the total cost of a call and providing better quality services by introducing faster and/or guaranteed network access, and better sound quality. The opportunity to achieve end-users QoE increment, lies in the traditionally considered Mobile Termination Rates (MTR) monopoly in mobile communications.

The total cost of each call placed by a subscriber of a MvNO is split into two parts. The first part determines the amount the caller's provider is charging to provide the service to the calling party. The second part includes the amount that the provider of the callee will charge the caller's MvNO to terminate the caller's call into the callee's network. End-users of mobile voice services have to pay both parts of these service costs. Thus, either the caller or the callee will pay for the termination fees. Figure 1.1 illustrates the present situation in the MTR ecosystem.

In countries where the Calling Party Pays (CPP) principle is applied, such as in European countries, MvNO subscribers rarely consider the termination cost that their operator is charging other networks when deliver- 


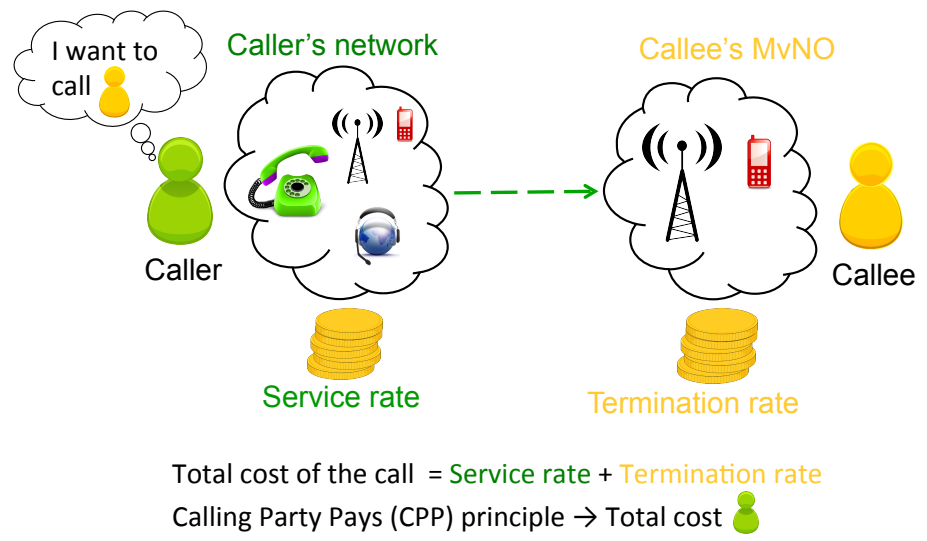

Figure 1.1: The MTR Ecosystem

ing an incoming call to them before they establish their contract, because MTR are not a key point when MvNOs advertise their products. Furthermore, a significant raise of MTR from MvNOs will increase the communication cost for all MvNOs subscribers. However, it will rarely have a negative impact and dissatisfy the customer base of a MvNO since its customers do not have to pay themselves the demanded MTR from their MvNO. The customer base of a single $\mathrm{MvNO}$ is significantly smaller compared to the set of total customers in every other voice services provider, such as competitive MvNOs, Fixed Network Operators (FNOs), or Voice-over-IP (VoIP) providers. Thereby, the majority of calls that a $\mathrm{MvNO}$ has to terminate in his network originate from foreign networks.

The MTR, since the early days of the mobile communications are considered to be a "de-facto" monopoly because ( 1 ) for many years an MvNO's revenue is coupled with high termination rates applied, and (2) MTR are decided by the caller's MvNO and the one to pay the termination rate (the 
caller cf. Figure 1.1) cannot influence it. Liberation of the Mobile Termination Rates monopoly so that multiple MvNOs will be able to deliver a call to a caller is not possible so far. Thus, the national telecommunication regulation authorities are usually regulating heavily MTR across the world (Europe [32][40], North and South America [47][9], and Asia [133]). Furthermore, the European Commission (EC) has opened a public consultation on the evaluation of the Termination Rates Recommendation (TRR) of 2009, as part of the EC's Digital Single Market (DSM) strategy [44]. The International Telecommunication Union (ITU) has investigated possible solutions to address this problem. There is a rich literature [34] on how regulation of this market is affecting it, as well as selected research on the topic of the proper selection of termination rates [25][121]. Furthermore, effort has been put into the analysis of business strategies which MvNOs follow concerning their MTR [62][93]. This thesis provides instruments needed for the MTR market liberalization where charging for QoE is a new business strategy for MvNOs.

However, since the initiation of mobile communications many issues on mobile terminal devices and network infrastructure have changed. Today, mobile networks have evolved in a way that their infrastructure does not support only voice services, but data as well [124]. Furthermore, mobile devices and networks operate in several bands. Last but not least, "today, your cell phone has more computer power than all of NASA back in 1969, when it placed two astronauts on the moon" [85]. In such environment this thesis shows that multiple MvNOs can terminate a call and lead to the Mobile Termination Rates monopoly liberation.

Therefore, this thesis considers the changes since the $2.5 \mathrm{G}$ mobile communication, analyzes the MTR ecosystem, and proposes an Auctionbased Charging and User-centric System called "AbaCUS", to overcome the monopoly obstacle of the MTR market. This thesis research questions, hypotheses, and contributions are illustrated in Figure 1.2. The key characteristic of AbaCUS is that the termination service is provided by the MvNO which maximizes the end-user's QoE. MvNOs are bidding in an auction and the winning MvNO (the one that will maximize the end-user's $\mathrm{QOE}$ ) is 


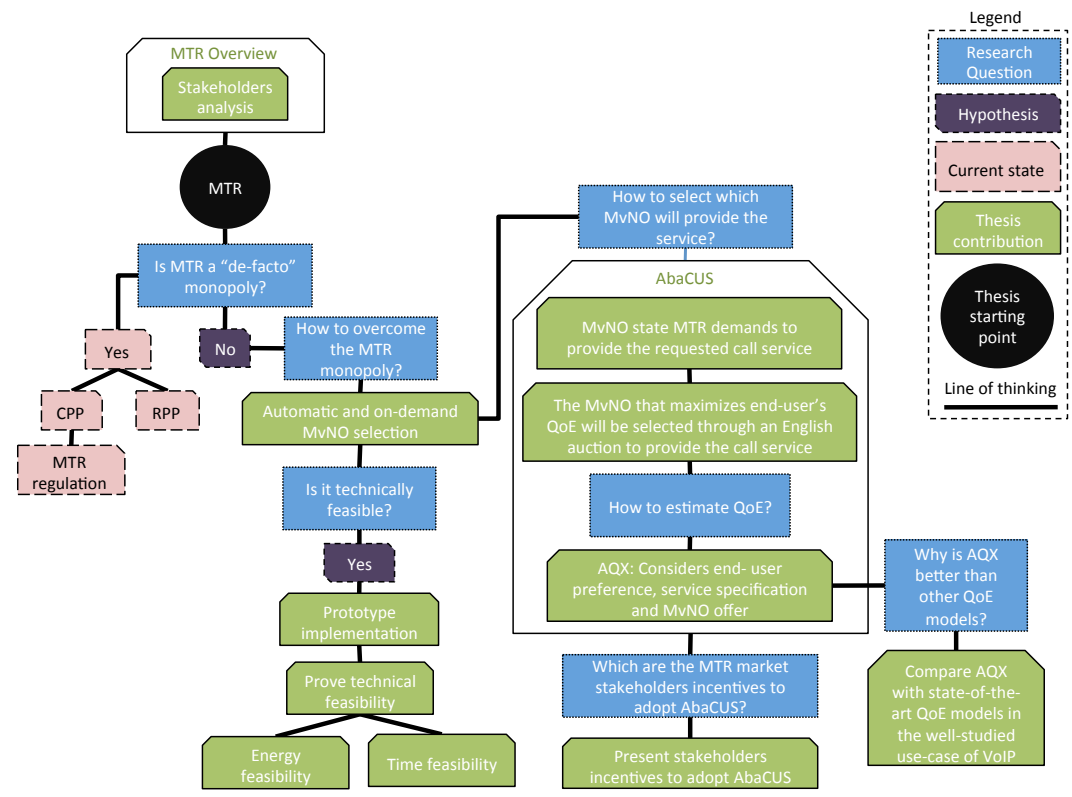

Figure 1.2: Thesis Overview

selected to provide the mobile call-termination service. Since QoE is used as a bidding metric in AbaCUS this thesis proposes a novel and generic $\mathrm{Ax}$ iomatic QoE (AQX) model to predict/estimate end-user's QoE, considering both technical and economic parameters, such as the sound quality and MTR price respectively.

AQX performance is compared against state-of-the-art QoE models in the well-studied use-case of VoIP to evaluate its performance in terms of prediction accuracy, to reveal existing QoE models weaknesses, and argue why a new QoE model is needed when charging for QoE is intended towards the Mobile Termination Rates monopoly liberation. Finally, this thesis implements prototypically an automatic and on-demand MvNO selection mechanism that is needed in a liberal MTR ecosystem. The usability of the implemented mechanism is evaluated in terms of its time and energy -efficiency to examine if such approach is technically feasible. 


\subsection{QoE}

In the Internet Technology (IT) ecosystem (end-users and Service Providers (SPs)) end-users' QoE is important information needed by SP to understand what and how end-users satisfaction is affected. However, end-user-satisfaction, which can be quantified by QoE metrics, cannot be easily measured like technical variables, such as bandwidth and latency because multiple and diverse variables technical and non-technical, such as bandwidth, latency, packet-loss, delay, and price, are affecting QoE simultaneously. QoE can either be estimated for a specific service through mathematical models, or it can be measured through an experimental setup [116][49].

For the VoIP scenario existing models, such as the exponential relationship connecting Quality-of-Service (QoS) parameters, called Interdependency of the QoE and QoS (IQX) Hypothesis [50] and the E-model [76] of the Telecommunication Standardization Sector of the International Telecommunication Union (ITU-T) [82], map technical variable values to Mean Opinion Score (MOS) [77]. However, those models either cannot consider simultaneously multiple parameters, or represent successfully only the average user since there are no user-specific preferences consideration. Unfortunately, the average user does not exist [57].

Measuring the impact of technical variables or resources priority-access on QoE of various services, demands an extensive feedback from end-users, when those variables change. Estimating QoE in a given scenario becomes harder when non-technical variables, such as price, need to be considered in addition to technical ones. The impact of technical variables, such as the maximum packet-loss that can be tolerated in VoIP, is specified in the VoIP protocol used. However, the end-user's willingness to pay for a call is not part of any protocol's specification. In any case detailed feedback that correlates all variables affecting $\mathrm{QoE}$ is needed by end-users for each service separately.

In this thesis the axiomatic mathematical QoE model AQX encapsulating user demands, user/service characteristics, and variable specifications, is proposed to formalize $\mathrm{QoE}$ prediction/estimation considering one or 
multiple and diverse variables. Furthermore, the output of QoE functions presented here can be normalized such that results will be compatible with the five-point scale MOS, proposed by the ITU-T [73], where 5 corresponds to "Excellent", 4 to "Good", 3 to "Fair", 2 to "Poor", and 1 to "Bad".

To tackle the challenge of QoE estimation when multiple technical variables are considered simultaneously, and to reveal the fact that the stateof-the-art QoE models [50] [76] fail to map accurately in every case enduser's QoE, a VoIP QoE measurement setup is essential to capture enduser's QoE in several VoIP scenarios. The setup requires input from multiple subjects to export scientifically valuable results. The data collected during these VoIP scenarios are used to define all necessary parameters of the AQX model in these VoIP scenarios. Such a calibration of the AQX model is essential to adapt it to the particular service and its technical and nontechnical conditions in which it is used. Furthermore, those AQX results achieved are compared with those results of the IQX Hypothesis and the E-Model. Thus, it is finally shown that AQX can capture more accurately end-user's QoE in VoIP scenarios.

\subsection{Mobile Termination Rates Monopoly Liberation}

In countries where the Calling Party Pays (CPP) principle is applied, the person that is dialing (caller) the phone number of a mobile user (callee) has to pay the MTR that the MvNO of the callee demands from the MvNO of the caller to deliver the call. Since the caller is not able to influence the MTR this market is considered to be a monopoly. To liberalize the MTR market a MvNO AQX-bidding process that will be used to define which MvNO maximizes the end-user's QoE is proposed in AbaCUS [2].

AbaCUS is a Global System for Mobile Communications (GSM) overlay, where the caller can select another MvNO operating in the area of the callee to deliver the call. Furthermore, AbaCUS describes in this thesis a process where the caller will define across a set of variables preferences concerning the sound quality, network access guarantees, and the pricing schema of the service. Thus, AbaCUS enables the competition in the MTR market [93] through an auction, since the caller defines preference select- 
ing among a predefined set of variables (sound quality and guaranteed network access), and additionally influences based on those preferences the MTR pricing schema.

\subsection{On-Demand MvNO Selection}

Assuming the knowledge of a MvNO that maximizes the end-user's QoE and AbaCUS in place, an automatic selection of the MvNO to be used is essential. However, the MvNO selection is possible on a User Equipment (UE) (a mobile device) through the respective User Interface (UI). Furthermore, mobile devices can be adjusted to select automatically the MvNO based on the strongest signal strength, among the list of those MvNOs the Subscriber Identity Module (SIM) card is allowed to be registered with. However, so far in modern mobile operating systems, such as Android and iOS, there is no available method in the public developer's Application Programming Interface (API) which allows for an automatic and on-demand selection of the MvNO by third-party and non-system applications.

This thesis presents an automatic and on-demand MvNO selection mechanism, that has been designed and implemented on the Android platform. For evaluation purposes the energy and end-to-end ( $\left.\mathrm{E}_{2} \mathrm{E}\right)$ time consumption while switching among MvNOs using this mechanism is evaluated and proven to be reasonable for an on-demand MvNO selection for the mobile call-termination service purposes. Thus, this thesis provides valuable input to (a) AbaCUS and (b) to the Android developers community.

\subsection{Thesis Contributions}

Motivated by these observations addressed above, this thesis makes the following contributions to the field of the QoE modeling, Mobile Termination Rates monopoly liberation, and automatic and on-demand MvNO selection:

1. Introducing $A Q X$, a generic axiomatic QoE model that considers simultaneously multiple technical and non-technical variables, as 
well as user preferences and user/service characteristics to predict and/or estimate QoE in terms of MOS. The model presented in this thesis is suitable for multiple IT domains, such as IP-based services, and similar but non identical services ranking. The generated MOS values can also be ITU-T MOS compliant if needed. Furthermore, this thesis is providing a calibration of the proposed model and shows that it outperforms state of the art existing models in certain VoIP scenarios, and presenting methodology that shows axiomatic ways of selecting the respective parameters of the QoE model.

2. Using end-user's QoE maximization and charging for QoE to terminate the "de-facto" Mobile Termination Rates monopoly, showing that the involved stakeholders can benefit from it in respect to the economic footprint of the mobile call-termination service liberation, and with additional instruments to regulator authorities that aim to overcome the mobile call-termination monopoly obstacle in the future.

3. Presenting an energy and time efficient automatic and on-demand MvNO selection mechanism, and proving that MvNO hopping is feasible. This finding can be used as an input not only for the MTR monopoly cancelation, but in other research approaches assuming this mechanism, such as electrosmog [126].

This thesis contributes in the following areas: QoE modeling [115][140]; QoE model calibration [137][138]; mobile call-termination monopoly termination [139]; and automatic/on-demand MvNO selection $[135][136]$. These contributions do represent important and innovative advancements in both research and Mobile Termination Rates monopoly liberation.

\subsection{Thesis Outline}

The remainder of this thesis is organized as follows. Chapter 2 presents related work on concepts that lay the technical foundation upon which this 
thesis stands; that includes charging for QoE in the IT domain, QoE modeling and QoE estimation and prediction, MTR monopoly and policies, and automatic and on-demand MvNO selection time and energy "cost".

Chapter 3 presents the axiomatic mathematic QoE model called AQX that considers simultaneously more than one technical and economic variables affecting end-users' QoE. AQX considers end-user's preferences and service characteristics, to define influence and importance factors for each variable involved in QoE estimation process.

Since AQX is a generic model that can be applied in many scenarios across the IT domain it needs to be calibrated and evaluated for its performance. Thus, Chapter 4 presents how AQX parameters can be selected, and how the model performs.

Chapter 5 presents the auction-based architecture to overcome the monopoly of the MTR market (AbaCUS). The stakeholders of this market and their incentives are verbosely presented. The variables affecting the call-termination service are illustrated, and the mapping of those variables to QoE is defined.

AbaCUS, among other research approaches, demands an automatic and on-demand energy and time -efficient MvNO selection mechanism which is presented in Chapter 6. The prototypically implemented MvNO selection mechanism for the Android platform in this thesis, is evaluated in terms of the MvNO selection time needed and its energy efficiency, to prove that such a mechanism is technically feasible. However, this mechanism is a work-around, since modern mobile Operating Systems (OSs) do not support such actions yet.

Finally, Chapter 7 concludes this thesis, summarizing contributions and key findings, drawing conclusions, and suggests future work. 


\section{2 \\ Related Work}

$\mathrm{T}$ HE QoE concept is relatively young but fast evolving in the IT domain. AbaCUS argues that charging for $\mathrm{QoE}$ is the path to liberate traditionally considered monopolistic markets in telecommunication, such as the MTR. Thus, it is essential to (a) examine in detail mobile voice services and the monopolistic MTR market, (b) get familiar with QoE, charging for QoE trends, and study state-of-the-art QoE models and outline its strengths and limitations when QoE-awareness for the Mobile Termination Rates monopoly liberation is attempted, (c) illustrate economic mechanisms to select on-demand a MvNO to terminate a call, such as auctions, and (d) investigate further automatic and on-demand MvNO selection demands. Thus, this chapter discusses technologies and models which are important building blocks of AbaCUS (cf. Figure 2.1).

\subsection{Mobile Voice Services}

In mobile voice services there are various stakeholders, such as MvNOs, regulators, and end-users who have a dual-role, since they act both as callers and callees. Regulators are governmental agencies that regulate businesses 


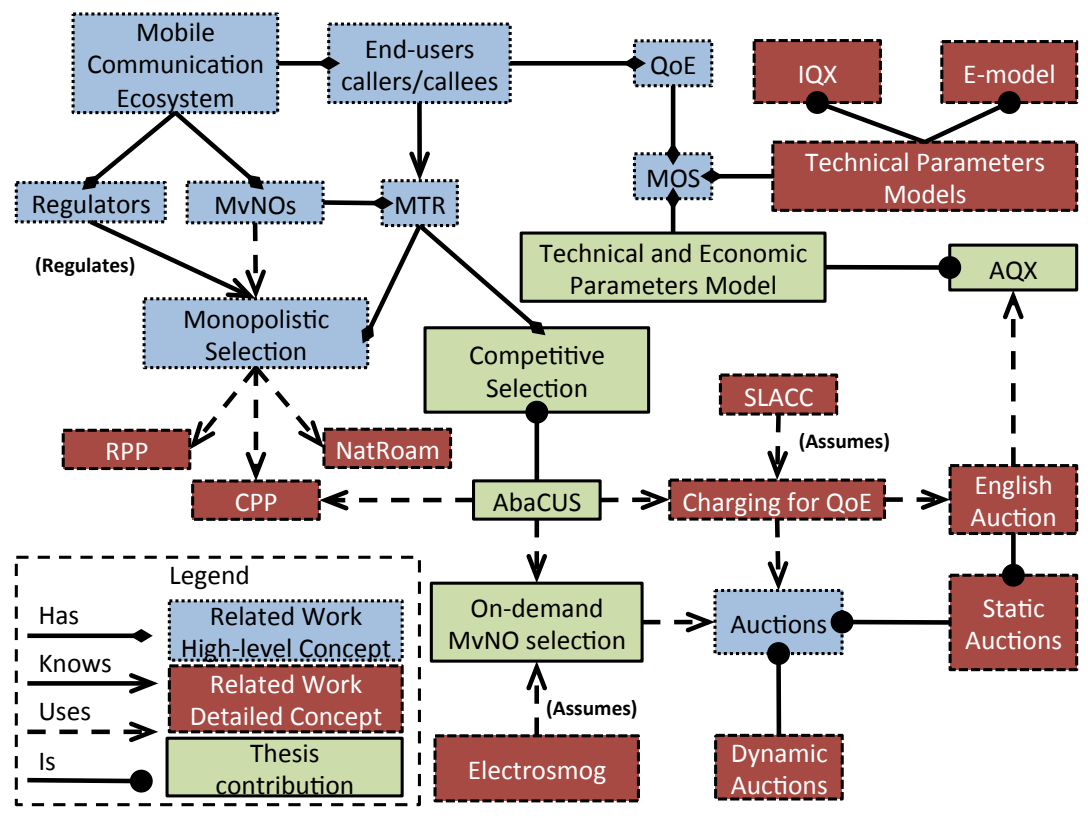

Figure 2.1: Related Work Overview

in the public interest and are units with administrative responsibilities that (a) create enforce and propose regulations and laws especially in monopolistic environments, such as the MTR, and (b) perform investigations and audits, and are authorized to fine the relevant parties or order certain measures in case it is needed. The existence of a regulation authority does not necessarily indicate a monopolistic market. Especially when thinking of an end-user acting as a caller, since MvNOs offer diverse services and pricingschemas and end-users are free to select a MvNO according to their needs and preferences. However, when thinking of the callee role the situation is different since there is only one MvNO that is able to reach the callee and provide the call termination service (route the call to the callee). The call termination service is coupled with MTR and thus this market is considered to be a "de-facto" monopoly and there is high regulation demand to deal with it. 
Since the early days of mobile communication there are many updates concerning the technology that could introduce competition in the MTR market. E.g., some UEs, such as dual-SIM devices allowed the end-user to select on-demand which MvNO to use for performing and receiving calls. Furthermore, the new smart phones have significantly more computational power compared to the early mobile UE, the new mobile Operating Systems (OSs), such as, Android, provide flexibility to build customized mobile software, and finally the new MvNO networks allow high-speed data. Those updates in mobile voice services make the MTR monopoly a marketdefined limitation.

\subsubsection{MTR MONOPOLY}

Since the early years of mobile communications, the scientific community as well as regulation authorities has invested a large effort [62][25][34], to reduce the negative effects of the MTR monopoly. However, the attempt to overcome negative effects of this monopoly is focused (a) on charging solutions mainly targeted at the paying party of MTR, or (b) on regulation rules that need to be enforced by respective regulation authorities at operational MvNOs. Thus, (1) the CPP principle with a strong price-regulator presence, (2) the Receiving Party Pays (RPP) principle, and (3) a National Roaming (NatRoam) approach, aim to eliminate negative effects of the monopolistic MTR market. However, in all cases the monopoly in this market still remains since only the MvNO of the callee can terminate his calls and profit from it.

\section{The CPP Principle}

The CPP principle is the most commonly used termination charging approach among MvNOs around the world, especially in European markets [68]. Within the CPP principle the caller has to pay call-termination charges and there is no contribution from the callee. This principle is the root of the monopoly problem in the MTR market. Thereby, strict regulations are applied to avoid the MvNOs speculation due to their dominant position. 
Despite the significantly lower MTR nowadays compared to the past decade, the percentage of MTR when considering the total call cost of a call still remains high (e.g., in March 2009 the regulations in India were amended so that termination rates for all types of domestic call, fixed or mobile, were reduced from the equivalent of some $0.006 \mathrm{US} \$$ per minute to 0.004 US \$ per minute [69]). Thus, the regulation demand for this market has not been decreased. However, regulating this market simply reduces the Mobile Termination Rates monopoly negative effects, while the heart of the problem is still beating, since only the callee's MvNO can terminate his calls and collect MTR for it. Defining and applying regulations is a timecostly procedure for regulators, which MvNOs often use to avoid/postpone a new regulation. Thus, a more efficient way to overcome negative effects of a monopolistic/regulated market is essential.

\section{The RPP PRINCIPLE}

In North America and some parts of Asia the RPP instead of the CPP principle is applied. In contrast to the CPP principle, in RPP the callee is asked to pay for the termination cost or in some cases to share a part of this cost with the caller. Initially this approach sounds fair, especially in the scope of the callee payment for the call-receiving service while being mobile and not located in the home network. Furthermore, a subscriber is free to compare termination rates of each $\mathrm{MvNO}$ and to make a choice before the establishment of a contract with a MvNO. Thereby, the MTR market initially appear to allow for competition. However, the question of how a callee could avoid payments for unwanted calls (e.g., advertisements, tele-sales, or polls) is raised. The answer is that it is the callee's responsibility to distinguish, which call is important and should be accepted and which should be rejected. This is only one of the RPP side effects [93] that feared to slowdown the mobile sector in the past. The RPP principle may add an extra degree of freedom in the mobile call charges, since MTR are not a part of the total cost that the caller has to pay. However, it is also adding a considerably big overhead for consumers such as the provider-selection decision, while considering the callee role. 


\section{The NatRoam Principle}

The NatRoam approach is partially used in countries of Latin America (e.g., TIM Brazil [26]). However, MvNOs that offer NatRoam services inside larger countries (e.g., within Brazil) offer a limited selection freedom among other MvNOs which are usually branches of the same company. Furthermore, switching to a different MvNO is allowed only in regions of the country where the subscriber's MvNO does not operate its own network infrastructure. Additionally, the user that is on NatRoam has also to pay for every incoming call exactly like in the international roaming case. Thus, NatRoam today has no influence on the MTR market.

Even in case that NatRoam was broadly allowed, or enforced by the regulator [39], it would be an overlay either in the CPP or in the RPP MTR collection principle. Thus, the price that the caller would have to pay in the CPP case would not be influenced by the caller, unless the caller could notify the callee to switch on a preferable MvNO. In the RPP case the callee would also have to establish a contract with each MvNO in the home location to be able to register the UE to any of them. In any case the Number Lookup Service (NLS) queries, prior to a call (for every call), through the Signaling System No. 7 ( $\left.\mathrm{SS}_{7}\right)$ network to the Home Location Register (HLR) of the MvNO, to find the MvNO that is currently reaching a Mobile Subscriber Integrated Services Digital Network-Number (MSISDN), would be mandatory. This procedure is costly since these NLS costs vary from 0.038 to $0.0038 €$ per look-up [94]. Therefore, an on-demand solution, which addresses the MTR monopoly, is proposed within AbaCUS.

\subsection{Auction Mechanisms}

Before the $17^{\text {th }}$ century auctions were not the common way to buy and sell goods, resources, or services [97]. All what the seller had to do was to set the price, and the buyer would either select to purchase the goods or not. In case that goods/resources were limited, the rule "first-come, firstserve" (FCFS) was usually applied. Nowadays, where the e-trading market is growing dramatically, auctions prove to be a powerful tool for competi- 
tion as well as for the increment of companies' profit. A considerably large number of people buy and sell all kind of goods using online trading Web sites (e.g., via Ebay [37]). Furthermore, companies often use auctions to sell their services (e.g., GoogleAdds [59]), while maximizing their profit. Governments use auction mechanisms to offer limited resources (e.g., spectrum or frequencies [48]).

Thus, during the last decades the scientific community as well as market leaders has put a lot of effort on creating and analyzing auctions with different rules. Those rules can be either simple or complex, and the auction can take place in one or multiple rounds. However, the target in every case is to increase either the revenue of the seller or the social welfare, avoiding at the same time problems like the bidder's curse [142]. Since the demand for real-time-decision-making auction mechanisms have increased in recent times, on-line auctions [66] [105] became popular. Many variations of well-known type of auctions (e.g., English [33], Dutch [121], or Vickrey's [21] auctions) argue to be optimal for goods, services, or resources, when on-line trading is addressed.

An alteration of the Vickrey-Clarke-Groves (VCG) auction without monetary compensation and a draw resolution mechanism is proposed in [139] for AbaCUS. The draw resolution mechanism also prevents a single bidder from overpowering other bidders in the auction and gives incentives to participate in some low revenue situations. However, VCG is not suitable in many dynamic situations as demonstrated in [123] where it is shown that the second-price sealed-bid auction, which is equivalent to VCG in setups with single items, is not truthful if losing bidders have the opportunity to win future auctions for the same item. This concept is called the "option value" associated with losing an auction in purpose. Further findings in [41] showed that the second-price sealed-bid auction does not only lack truthfulness but fails to yield an explain efficient outcome in some situations.

A social welfare maximizing generalization of the VCG mechanism $[31][61][145]$ for services such as the mobile call, the Dynamic Pivot Mechanism (DPV), has been proposed in [23] and examined for AbaCUS purposes in [41]. In DPV after each bid the expected payment for each 
MvNO coincides with the dynamic externality cost the MvNO imposes on the rest competing MvNOs. This is done as in the classic VCG mechanism by calculating for each MvNO the optimal allocation when the winning $\mathrm{MvNO}$ is not present in the mechanism. Therefore, each MvNO is willing to truthfully report the next bid after every bid. The mechanism is modeled in discrete time and all MvNOs share a common discount factor.

The private information is the MvNO's perception of the future payoff path based on the public history of allocations and his private history of realized calls. The MvNO's private type evolution is modeled as a Markov decision process in discrete time. In [28] DPV is extended to work in domains with a dynamic population and dynamic information, such as the mobile calls environment where the population of MvNOs can change because some are unreachable or without available resources. This is a powerful extension for many applications, such as the mobile calls environment. However, its long run-time execution make it is unusable in practice into the call termination domain, where real-time decision is needed.

Thus, in the specific setting of AbaCUS since a single item, a call, is being auctioned (cf. Section 5.5), MvNOs compete in a static auction (i.e., English auction), where QoE is the bidding metric, and the MvNO that maximizes end-users $\mathrm{QoE}$ is selected to provide the mobile termination service and benefits from collecting MTR.

\subsection{Flexible MvNO Selection}

Automatic and on-demand MvNO selection might be technically possible since there are new mobile network capabilities (mobile data), significantly more computationally capable UEs (smart phones), and no physical barriers preventing a UE to be registered to any MvNO. Furthermore, a liberal on-demand MvNO selection might also be beneficial for end-users and MvNOs, in economic and resources availability utilization terms. Thus, the market-defined limitation of bounding the end-user with one MvNO needs to be challenged. 


\subsubsection{Apple Patent}

The first attempt towards an on-demand Mobile Network Operator (MNO) selection approach is introduced by Apple in 2006 in a patent [46]. In Apple's approach the MNO that will provide a service to the end-user such as an outgoing call is decided by Apple. MNOs are bidding in an auction initiated by Apple who acts as a Mobile Virtual Network Operator (MVNO). The Apple MVNO selects which MNO will provide the service. On one hand, in this scenario the Apple MVNO benefits from selecting the lower price that the actual MNO offers to deliver the service. On the other hand, the Apple MVNO will charge a standard fee to the end user. In other words, at [46] Apple attempts to patent the right for competition in the MTR market! In Apple's patent the auction takes place between MNOs and the Apple MVNO. However, there is an important business requirement missing in [46]. Additionally to the price, a set of technical preferences, such network resources access guarantees and the QoS of the call cannot be defined by the end-user.

\subsubsection{Apple SIM CARD}

To select a MvNO on-demand a SIM card that can be registered in every MvNO is needed. Apple SIM [149] partially overcomes this obstacle since the Apple SIM card can be issued by and used with selected MNOs. However, there is not a mechanism that allows the User Equipment (UE) to switch from a MNO to another in an automated way, on-demand, and without any interaction. Apple SIM supports only some MNOs in the US and the UK [149]. Additionally to the limited number of supported MNOs by the Apple SIM card, in the early days of the commercial use of such SIMs one of the market leader MNOs in the US had the decision to permanently lock Apple SIM when activating for first time, requiring the purchase of a new Apple SIM to use a different carrier. AbaCUS presents clear benefits for MvNOs as an incentive to participate in the competitive termination service market proposed in this thesis. 


\subsubsection{Flexible MNO Selection Demands}

A novel work to make use of an automatic and on-demand MNO selection is presented at [126]. A network switching selection model and its algorithms minimizes the non-ionizing radiation of devices during use. The key goal is to minimize the exposure of the mobile user to electromagnetic radiations, while still providing a certain QoS level. Within a proof of concept [126] validated the model and its algorithms. Due to the fact that the Android Application Programming Interface (API) does not provide for a mechanism to force switching from one MvNO to another, the user has to manually select a network. This takes a lot of time, because the provided mechanism by the Android platform searches first for all available networks, which is a time consuming operation. This time overhead makes it impractical to apply a MvNO selection algorithm.

The available MvNOs in a country are well known and do not change often. Thus, to avoid the MvNO searching delay in the prototyped mechanism that was implemented in this thesis, this operation is skipped while a MvNO is selected. Available MvNOs in a location are stored in a list and when needed the respective $\mathrm{MvNO}$ is selected from that list. Nevertheless periodic updates of that list, e.g., daily, when an application or a process that uses automatic MNO selection starts, or when a user moves in a predefined area, are essential to ensure that all currently available MvNOs are stored in the list. Thus, the proposed MvNO selection mechanism in this thesis here can be used for the non-ionizing radiation minimization purpose as well. The evaluation of this mechanism, in terms of energy and time consumption per MvNO switching can define a threshold of a maximum number of hops allowed in the non-ionizing radiation minimization MvNO selection approach, so that the electrosmog reduction approach remains both realistic and energy efficient.

\subsection{QoE}

QoE is a subjective concept that shows the end-user's perceived quality of a service and it can be either measured, or estimated. To measure QoE 
Table 2.1: The MOS Scheme Recommended by the ITU-T [73]

\begin{tabular}{|c|c|}
\hline MOS Value & Quality \\
\hline \hline 5 & Excellent \\
\hline 4 & Good \\
\hline 3 & Fair \\
\hline 2 & Poor \\
\hline 1 & Bad \\
\hline
\end{tabular}

subjective measurements are needed. To capture the end-user's experience in QoE experiments, the five-point opinion scale recommended originaly for VoIP by the ITU [77] is often applied for other services as well, such video streaming [63]. This opinion scale is used in many QoE-related research and determines an excellent basis for comparing results. This scale defines scores from one to five, while each score defines a certain meaning. The ITU recommendation [73] assigns to each score an English word (cf. Table 2.1). Measurements that aim to "capture" end-user's QoE require end-user's feedback and thus are time-intensive, costly, and cannot measure QoE in real-time. Because of these drawbacks, objective QoE has been defined as automated procedures involving algorithms (QoE models) that approximate subjective $\mathrm{QoE}$ without requiring active ratings by users.

\subsubsection{QOE MODELS}

When end-users are asked to rate the performance of a service in a given scenario, there is a certain alignment on results. E.g., the quality of a conversation of a VoIP call that was performed with a specific codec is mapped to a specific MOS [77]. Furthermore, while comparing similar services with different variables, such as conversations on a VoIP network with different codecs, the MOS can be used as a comparison metric. Thus, a QoE model which is either an empirical or an axiomatic MOS calculation algorithm, addressing services affected by diverse variables (technical and economic), can be used for service-comparison purposes. There are several QoE models presented in [4]. Table 2.2 summarized the state-of-the-art QoE models 
Table 2.2: QoE Models

\begin{tabular}{|c|c|c|}
\hline QoE Model & Type of Service & Variables \\
\hline \hline IQX [65] & VoIP & Single (technical) \\
\hline E-model [76] & Voice Services & Multiple (technical) \\
\hline $\begin{array}{c}\text { Global Correlation Model } \\
\text { (GCM) [20] }\end{array}$ & Video Streaming & Multiple (technical) \\
\hline PARQUE [147] & Internet Services & Multiple (mixed) \\
\hline AQX [140] & Service-independed & Multiple (mixed) \\
\hline
\end{tabular}

that are designed to estimate $\mathrm{QoE}$ in specific scenarios and/or considering in most cases technical variables only, and includes AQX which is a newly designed generic QoE model, that can be used for QoE estimation purposes in multiple services while considering multiple and diverse parameters, and thus it is also usable for service comparison purposes.

\section{IQX НуротнеSIS}

The exponential Interdependency of QoE and QoS model (IQX Hypothesis) assumes an exponential relationship between $\mathrm{QoE}$ and $\mathrm{QoS}$ [50][65]. $\mathrm{QoE}=\Phi\left(I_{1}, I_{2}, \ldots, I_{n}\right)$ in $[65]$ is a function of $n$ influence factors $I_{j}, 1 \leq$ $j \leq n$ (the equivalent term for influence factor is variable in AQX, since the term influence factor used for different purposes in this thesis). To motivate the fundamental relationship between $\mathrm{QoE}$ and an impairment factor (antitonic variable in this thesis) corresponding to QoS, the packet loss probability $p_{\text {loss }}$ is examined in the VoIP scenario. Similar to [120] $\mathrm{QoE}=\alpha \cdot e^{-\beta \cdot p_{\text {loss }}}+\gamma$ assumed to be an exponential function. Thus, fitting the curve of ITU-T-compilant MOS (c.f. Table 2.1) measurements concerning the Internet Low Bitrate Codec (iLBC) voice codec ( 400 bits each $30 \mathrm{~ms})$ at [65] provided the numerical values for the parameters $\alpha=3.01$, $\beta=4.473$, and $\gamma=1.065$ (cf. Figure 2.2).

IQX is not meant to be used as a service comparison tool, thus, it is not possible to be used for charging for QoE purposes. In this thesis the concept 


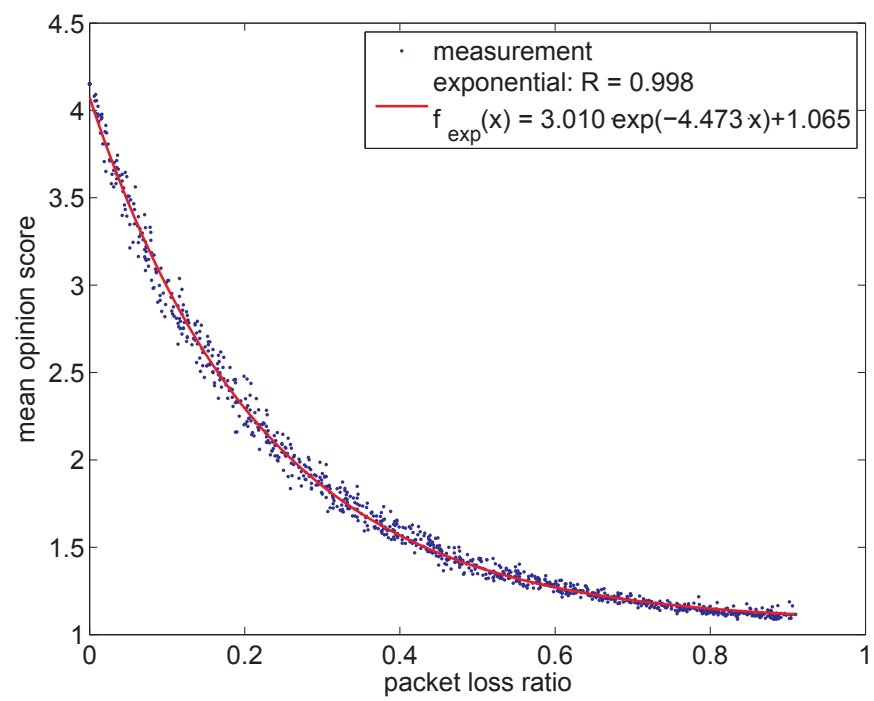

Figure 2.2: QoE Mapping Function of Packet Loss Ratio in the IQX Hypothesis [50]

of IQX is extended to more than one variable that can also affect QoE not only negatively but positively as well, so that a high level comparison among different services expected QoE can be facilitated. Furthermore, the mathematical model that is proposed, provides more flexibility with respect to the influence that a variable, e.g., $p_{\text {loss }}$, might have in different services, e.g., different VoIP codecs, or for different users, e.g., business, and home users. The later is achieved by introducing influence factors for each variable affecting the overall QoE. The influence factor is a power operator that applies on variables and shows how fast $\mathrm{Q} o \mathrm{E}$ will be affected at a given fluctuation of each variable. Additionally, the concept of the Expected Variable Value $\left(\mathrm{eV}^{2}\right)$ for each variable is introduced here. The role of the $\mathrm{eV}^{2}$ is fundamental in the selection and calculation of $\alpha, \beta$, and $\gamma$ that result from the curve fitting in IQX. Finally, IQX is extended in AQX by introducing the concept of the Expected MOS (eMOS), which is a value that is less than the maximum possible MOS. The latest facilitates the assumption that a certain level of QoE can be maintained even if one variable, e.g., ploss, changes. 


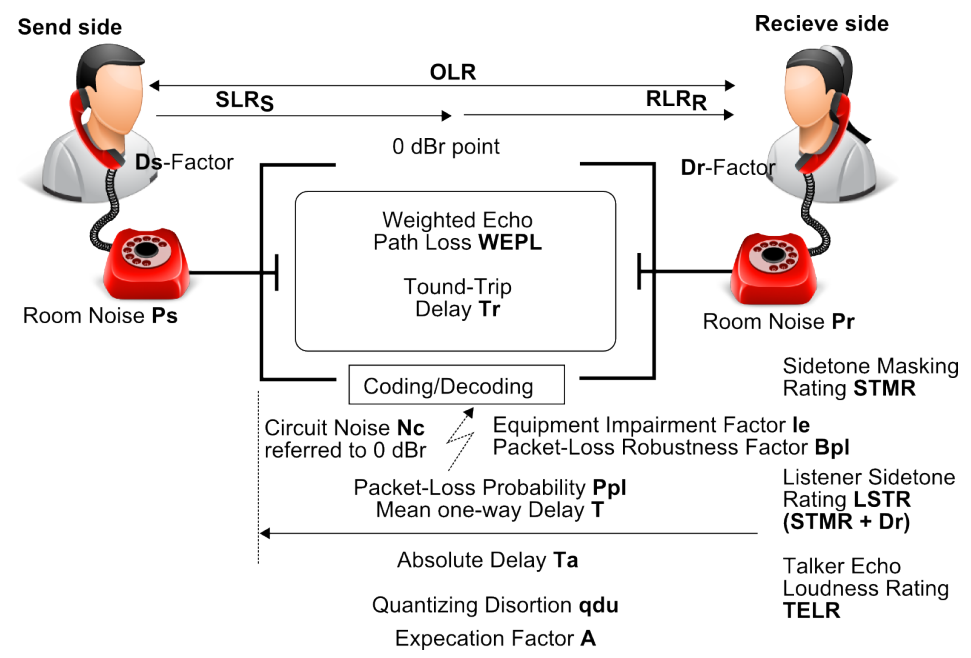

Figure 2.3: Reference Connection of the E-Model [76]

\section{E-MODEL}

The E-Model [76] is a transmission planning tool that can be used to predict $\mathrm{QoE}$ for a typical telephone user in an End-to-End ( $\left.\mathrm{E}_{2} \mathrm{E}\right)$ conversational scenario. The model takes a wide range of transmission variables into account and it can be used to assess the voice quality of wired and wireless services, based on circuit-switched and packet-switched technology [79]. The output of this model is — in contrast to other models — not in form of MOS values. The E-model uses the Transmission Rating Factor $R$ as output, which can be transformed into MOS and, therefore, it becomes possible to compare the E-model to other QoE models. The E-Model uses mathematical algorithms based on the analysis of a large number of subjective tests with a wide range of transmission variables. These algorithms can transform transmission variables into so called "impairment factors". According to the E-model tutorial [79], five impairment factors are used to calculate the $R$ value.

- Ro: Expresses the basic signal-to-noise ratio, including various noise sources, such as circuit noise and room noise. 
- Is: This term takes impairments into account that exist more or less simultaneously with the voice signal, such as, (a) too loud speech level, non-optimum Overall Loudness Rating (OLR), (b) non-optimum Side Tone Masking Rating (STMR), and (c) impairment caused by quantizing distortion.

- Id: This factor represents all impairments that are caused by too long absolute delay and potential echo effects on both talker's and listener's side.

- Ie: Equipment impairment factor represents impairments that are caused by the respective codec used and packet-loss.

- A: The advantage, or expectation factor, considers the advantage of service access. E.g., a user in a region which is hard to provide connectivity, such as regions where a satellite link is needed, expects a lower quality, and therefore, tolerates more impairment.

Equation 2.1 considers all impairment factors to calculate the $\mathrm{R}$ value $[76]$ :

$$
R=R_{\circ}-I_{s}-I_{d}-I_{e}+A
$$

All impairment factors are calculated through algorithms that take several transmission variables as input. An overview over all variables being used for the calculation is illustrated in Figure 2.3, where a telephone connection and all impairment factors affecting the quality of the conversation according to the E-Model is illustrated. As can be seen in Figure 2.3 the E-Model has a high complexity considering many different parameters. A detailed calculation of each impairment factor can be found in the ITU-T recommendation G.107 [76]. A question answered in this thesis is if the QoE model proposed here (AQX), which has a comparatively low complexity can keep up with the E-Model.

\subsubsection{Charging For QOE}

There is significant work already done towards charging for QoE [50][116][119]. The main focus is on QoE charging (a) for IP-based ser- 
vices [117], (b) for Cloud Provider (CP) services selection methods, and (c) services in the show business [ 1111$]$. However, there is not available so far a generic enough QoE model that can encapsulate economic and technical parameters, such as they can be used to charge for QoE in the telecommunication domain. Furthermore, a concrete scenario where charging for QoE can be applied in telecommunication is missing from the literature.

Comparing cloud services, such as performance cloud servers, load balancers, and file servers offered by different CPs is not trivial. Large CPs, such as Rackspace [114], GoGrid [56], and Amazon Elastic Compute Cloud (EC2) [5], offer comparable services with slightly different characteristics concerning Central Processing Unit (CPU), Random Access Memory (RAM), available bandwidth, OS, and charging schemes. There is not always an exact mapping of services across those CPs. Thus, it is hard for the end-user to decide which is the right product and CP to choose, when considering main characteristics and constraints of a service. A mathematical model that can generate an estimated MOS for each product and $\mathrm{CP}$, receiving as an input minimal service demands and end-user priorities concerning each variable, will be a powerful tool for end-users when CPs' products comparison is requested. AQX is shown to be suitable for CPs service's-value indexing.

When a comparison between diverse but similar products, such as products offered by CPs, is needed the only constant parameter is the end-user's preference and services' constraints and demands. Thus, considering the latest to calculate the expected QoE before concluding the selection of a specific product is essential. In the CPs scenario, the Service Level Agreement support system for Cloud Computing (SLACC) [96] approach suggests the existence of an SLA negotiation process (cf. Figure 2.4). CPs offer various pre-formed SLAs to end-users. However, end-users might have demands concerning the price and technical parameters, which are not satisfied by any predefined SLA. Thus, during the SLA negotiation process the end-user could advertise service demands. The CP could consider enduser's preferences input and try to maximize the estimated QoE while minimizing the SLA violation probability. Before the CP could make an SLA 


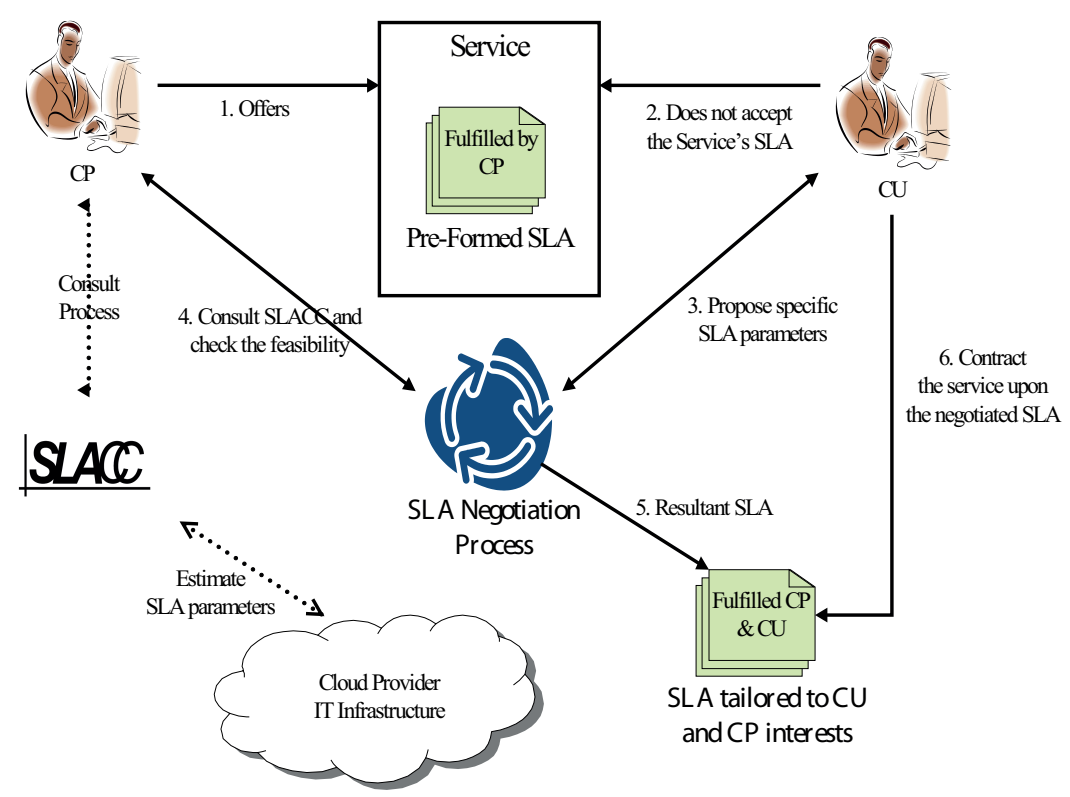

Figure 2.4: SLACC Solution Overview [96]

offer to the end-user, SLACC, which is a decision support system for CPs, would examine if such an SLA can be fulfilled. Thus, a mathematical model like AQX can be used for the estimated QoE maximization process.

\subsection{Contribution Opportunities}

While the field of QoE and mobile communications has received significant attention from the research community, the related work research presented in this chapter has revealed the following:

1. Encapsulating simultaneously, multiple technical and non-technical parameters in QoE models is missing.

2. An architecture that liberates the Mobile Termination Rates monopoly without creating new monopolistic markets is not in place. 
3. A mechanism that enables automatic and on-demand MvNO selection in a time and energy-efficient manner is needed to achieve the Mobile Termination Rates monopoly liberation.

Having identified the above shortcomings, and in direct relationship with the four observations made in Section 1.4, the following opportunities for scientific contribution in the area of QoE and mobile communications have been revealed:

1. create a generic axiomatic QoE model that encapsulates multiple and diverse parameters. The outcome of the model can be used to facilitate charging for $\mathrm{QoE}$;

2. define a methodology of selecting the parameters of the QoE model and identify further potential improvements; and

3. present an architecture that can overcome the MTR monopoly, as well as a time and energy -efficient automatic and on-demand MvNO selection mechanism.

Hence, chapters $3,4,5$, and 6 present a detailed description of the above contributions. 


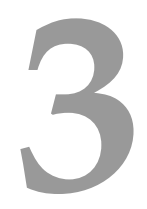

\section{AQX: Axiomatic QoE Model}

UAlity-of-Experience is a user-centric and service-specific concept reflecting the end-user satisfaction of a service while considering various technical variables, such as latency, bandwidth, or jitter, in VoIP services of the telecommunication field [131], or in video streaming of the entertainment field [27][99][144]. Furthermore, the QoE concept can also be used when considering pricing for IP-based services $[90][117][119][64]$, because the price of a service affects the overall enduser experience. Thus, $\mathrm{QoE}$ can be affected by (a) diverse technical variables and (b) by economical/non-technical variables.

In the IT ecosystem such variables are usually defined in the SLA between the SP and its customer. When one or more of these variables do not meet the agreed level, an SLA violation is occurred. However, an SLA violation does not mean that the end-user dissatisfaction cannot be avoided. There are certain actions that a SP can take, such as offering the service at a lower price, or offering a service upgrade, such as a higher bandwidth for the same price, to maintain the QoE of an end-user at a certain level of satisfaction. To prevent a potential decrement of $\mathrm{QoE}$ in case of an SLA violation, it is important to know which variables and how exactly they affect the end-user's QoE. A proper adjustment of involved variable(s) on the QoE 
might counterbalance the incident that caused the SLA violation in terms of the end-user satisfaction. However, such a process needs a formally complete and generic overview of the $\mathrm{QoE}$ concept that is missing nowadays. The need to illustrate QoE contributed to the creation of standards, such as the MOS $[77][73][78]$. The MOS reflects the end-user satisfaction at a numerical scale where the higher the score is the higher the end-user's satisfaction is and vice versa. However, since the MOS defines a subjective value, a complete and formal calculation of the MOS while considering all variables that might affect the $\mathrm{QoE}$ is the missing piece toward the precise user satisfaction demands estimation.

This thesis formalizes QoE in AQX which considers the agreed values of a set of variables described in the SLA and the measured values of those variables when/while a service is provided. Such measurable information that defines the $\mathrm{QoS}$ is used to calculate a MOS-normalized value that represents the end-user's QoE. Furthermore, this thesis defines as an SLA violation: the situation where there is no possible adjustment for any variable(s) that can result into an agreed upon MOS score between the enduser and the SP. Thus, QoE calculation equations in this thesis here are used to export MOS results once the set of the AQX model parameters and variables is defined.

\subsection{QOE Formalization IN AQX}

AQX assumes the following key QoE parameters, which are (a) servicespecific, (b) user-centric, and (c) can be influenced by the SP to formalize QoE. (1) The minimum and maximum values of a variable (e.g., price, bandwidth, or latency) affecting QoE; (2) the expected, or agreed in the SLA values of each variable; (3) the importance and the influence factor of each variable for each service; and (4) the desired codomain of the QoE functions. Thus, below are summarized the axioms of the AQX model that serve as a starting point of reasoning, to be accepted as true without controversy [24]. 
1. To predict/estimate $\mathrm{QoE}$, the first action needed is to identify all variables that affect QoE and can be measured, or can be estimated.

2. There are two types of variables affecting QoE: (a) Isotonic Variables (IV) which have an opposite effect in MOS and thus must be investigated separately. The more you have the better it is (e.g., bandwidth). (b) Antitonic Variables (AV). The more you have the worst it is (e.g., price). Further IVs and AVs examples can be seen in Table 3.1.

3. For each service there are two values per variable that define (a) the worst, and (b) the best possible values.

4. For each service and end-user there is an ideal/ desired/expected/agreed value of each variable $\left(\mathrm{eV}^{2}\right)$ that shows that the end-user is satisfied. This value is between the best and the worst variable values. However, in some cases the end-user can be satisfied even more, such as when receiving further price discount.

5. The fluctuation of the value of each variable might affect differently each end-user at a given service. The QoE effect of the fluctuation is expressed via the influence factor of a variable. The influence factor $(m)$ can be different for values below and above the $\mathrm{eV}^{2}\left(m^{-}, m^{+}\right)$.

6. Each variable affecting QoE can have different importance $(w)$.

Table 3.1: IV and AV Examples

\begin{tabular}{|l|l|}
\hline \hline \multicolumn{1}{|c|}{ IV } & \multicolumn{1}{c|}{ AV } \\
\hline up/down -link bandwidth & \\
data volume consumption & price \\
Short Message Service (SMS) & latency \\
calling time & jitter \\
sound quality & packet loss \\
resources access priority & delay \\
resources access guarantees & \\
\hline
\end{tabular}


A precise QoE formalization demands a mathematical model that is able to consider multiple and diverse variables, such as network access guarantees, network access priority, price, and bandwidth that can affect the enduser QoE positively or negatively on a given situation. Furthermore, each variable might affect QoE in a different way in each scenario. Additionally, QoE strongly depends on the end-user since each person might have different demands and priorities concerning the same services. The high-level formalization of the QoE is illustrated in Equation 3.1.

$$
\text { QoE }:=f(\text { user, service, variables, mood, context) }
$$

Although a given end-user's mood at a certain time can be considered as a variable that affects $\mathrm{QoE}$, it is not possible to be influenced by a SP. AQX is designed to model $\mathrm{QoE}$ that can be influenced by variables that are within SPs influence-zone, such as technical and economic variables. Furthermore, when QoE formalization is needed it should be treated as a bounded concept since a user cannot be infinitely satisfied or dissatisfied. E.g., doubling the price of a service that is not affordable, will not satisfy less the end-user since the service was already not accessible due to high cost. For instance, doubling the bandwidth of an average broadband plan without increasing the price, will not affect QoE proportionally, since services such as browsing, video streaming, or VoIP, perform equally well without such a bandwidth increment. Thus, it is assumed that there is a minimum $\mu$ and a maximum $M$ QoE that can be represented, without any loss of generality, by the positive parameters, $\mu, M \in(\circ, \infty)$, where $\mu, M \neq \infty$. Since $\mu<M$, let $h$ (cf. Equation 3.2) be a positive parameter that represents the codomain of the QoE function (cf. Equation 3.1).

$$
h=M-\mu>0
$$

Figure 3.1 illustrates $\mathrm{Q} o \mathrm{E}$ of the end-user for variables that can influence QoE positively or negatively when fluctuating. The $Y$-axis shows the MOS of a variable in the interval $h$ and the $X$-axis the normalized value $x$ of each variable. The value $e_{\circ}$ on the $Y$-axis is the MOS that corresponds to the ex- 


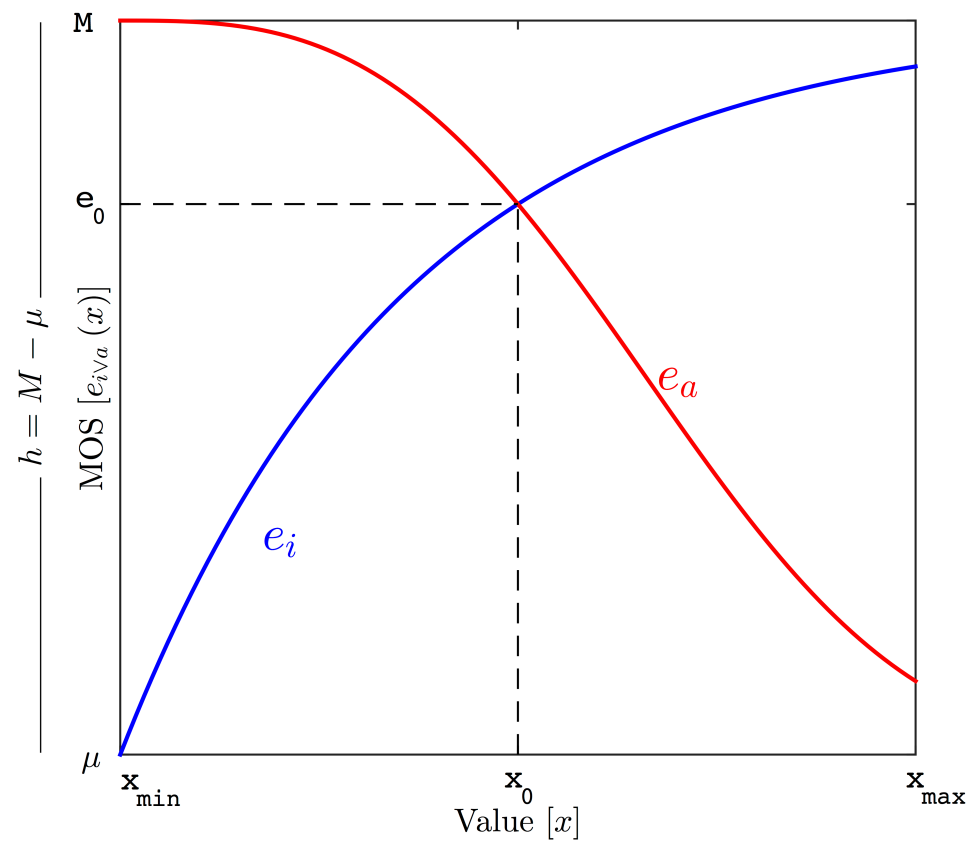

Figure 3.1: MOS Evolution for IV $\left(e_{i}\right)$ and $\operatorname{AV}\left(e_{a}\right)$

pected, agreed, or defined in the SLA value $x_{\circ}$ of each variable. Thus, let $e_{\circ}$ be the eMOS and $x_{\circ}$ the $\mathrm{eV}^{2}$ (cf. Section 2.4.1). On one hand, the $e_{i}$ curve reflects the MOS of a variable, such as bandwidth. Such variables while increasing to a maximum value $x_{\max }$, imply a QoE increment to the maximum MOS value $M$. Those variables in AQX are termed Isotonic Variables (IV). Furthermore, when the value of an IV is minimum $x_{\text {min }}$, the MOS value is also minimum $(\mu)$. On the other hand, the $e_{a}$ curve reflects the MOS of a variable, such as the price of a service. Such variables, contrary to IV, while increasing to a maximum value $x_{\max }$, imply a QoE decrement to the minimum MOS $\mu$. Those variables are termed Antitonic Variables (AV). Last but not least, when the value of a $\mathrm{AV}$ is $x_{\min }$ the MOS is M. E.g., when a service is provided without charging the $\mathrm{QoE}$ is maximum (cf. Equations 3.3 and Equations 3.4). 


$$
\begin{aligned}
e_{i}\left(x_{\min }\right) & :=\mu \\
e_{i}\left(x_{\text {max }}\right) & :=M \\
e_{a}\left(x_{\min }\right) & :=M \\
e_{a}\left(x_{\text {max }}\right) & :=\mu
\end{aligned}
$$

The IV or AV characterization of a variable describes the nature of the argument variables in Equation 3.1. The $x_{\min }$ and $x_{\max }$ values are related to the argument service in the same equation. E.g., the maximum throughput attainable is governed by the mobile link technology.

As an illustration, the average sector throughput in Long Term Evolution (LTE) multiple-input and multiple-output (MIMO) $4 \times 4$ with 20 $\mathrm{MHz}$ bandwidth, the most deployed form of LTE, provides a maximum of 12.7 Mbps uplink and 50.1 Mbps downlink throughput [100]. Thus in this case the minimum ( $\min$ ) and maximum ( $\max$ ) values concerning the uplink bandwidth (ULB) of the LTE MIMO $4 \times 442 \mathrm{MHz}$ technology are: $U L B_{\text {min }}=o$ Mbps and $U L B_{\max }=12.7 \mathrm{Mbps}$. Similarly, for the downlink bandwidth $(D L B)$ the $\min$ and $\max$ values are: $D L B_{\min }=0 \mathrm{Mbps}$ and $D L B_{\text {max }}=50.1 \mathrm{Mbps} . U L B_{\text {min }}=D L B_{\text {min }}=0 \mathrm{Mbps}$ because this means no connectivity.

Finally, the value $x_{\circ}\left(x_{\min }<x_{\circ}<x_{\max }\right)$ reflects the effect of a variable that affects $\mathrm{QoE}$ in a specific service (cf. service in Equation 3.1). However, the value $x_{\circ}$ of a variable might not necessarily be related to the technology, such as the bandwidth of LTE, due to the physical characteristics of the technology. $x_{\circ}$ might also be dictated by specific service demands, such as minimum bandwidth requirements. To illustrate, Hulu, a website and over-the-top (OTT) subscription service offering ad-supported on-demand video-streaming, recommends a downstream throughput of at least 1.5 Mbps for smooth playback experience of Standard Definition (SD) 48 op videos [67]. Thus, the value $x_{\circ}$ for bandwidth in this case is defined by the service minimum demands and not by the broadband technology (e.g., LTE, or $\mathrm{xDSL}$ ). 


\subsection{Single-VARiable QoE Functions}

The IV and AV QoE functions defined here are formalizing QoE considering a unique variable that is either IV or AV. A generic QoE function that encapsulates multiple variables, IV, and/or AV, is presented in Section 3.4. Without any loss of generality unity-based normalization of the IV value $x$ is assumed (cf. Equation 3.5), to enable plotting the MOS of multiple valuables in one graph.

$$
x:=\frac{x-x_{\min }}{x_{\max }-x_{\min }} \forall x \in \mathbb{R}
$$

\subsubsection{QOE Function}

The function $[0,1] \stackrel{e_{i}}{\rightarrow}[\mu, M]$, (cf. Equation 3.6) is defined to correspond to the QoE of an IV, such as bandwidth. The $e_{i}$ function that is illustrated in Figure 3.2 behaves like a step function for large values of $m$, like an exponential function for $m=1$, and like a constant function for $m \ll 1$. Furthermore, for $m \in(1,3], e_{i}$ changes in a smooth way. The influence factor $m \in[0, \infty)$ of a variable denotes the end-user's tolerance in fluctuations of variable's value $x$. E.g., services that demand not less than a specific amount of bandwidth will see a high influence factor, while if the bandwidth does not affect QoE a lot, the influence factor will be low. Finally, parameters $h$ and $\mu$ of Equation 3.6 are obtained through Equation 3.2 and Equations 3.3. Thus, Equation 3.6 is defined (a) to satisfy AQX axioms, and (b) to be able to model multiple QoE curves that behave almost as constant, step, or exponential functions. Equation 3.6 can be used in VoIP scenario to model bandwidth (a continuous variable) as well as guaranteed network access (a binary variable: yes/no). Thus, it is applicable in diverse scenarios and variables.

$$
e_{i}(x):=h \cdot\left(1-e^{-\lambda \cdot x^{m}}\right)+\mu
$$

The Expected Variable Value $\left(\mathrm{eV}^{2}\right) x_{\circ}\left(0<x_{\circ}<1\right)$ is defined to be the Expected MOS (eMOS) $e_{0}$, where $\mu<e_{0}<M$. Thus, given this 
assumption the parameter $\lambda$ is presented in Equation 3.7.

$$
\begin{aligned}
& e_{i}\left(x_{\circ}\right):=e_{\circ} \\
\Rightarrow & e_{\circ}=h \cdot\left(1-e^{-\lambda \cdot x_{\circ}^{m}}\right)+\mu \\
\Leftrightarrow & \frac{e_{\circ}-\mu}{h}=1-e^{-\lambda \cdot x_{\circ}^{m}} \\
\Leftrightarrow & \frac{h-e_{\circ}+\mu}{h}=e^{-\lambda \cdot x_{\circ}^{m}} \\
\Leftrightarrow & \ln \left(\frac{h-e_{\circ}+\mu}{h}\right)=-\lambda \cdot x_{\circ}^{m} \\
\Leftrightarrow & \lambda=x_{\circ}^{-m} \ln \left(\frac{h}{h-e_{\circ}+\mu}\right)
\end{aligned}
$$
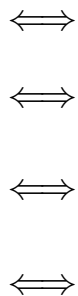

Replacing $\lambda$ above in Equation 3.6 results in Equation 3.8, which is the QoE function that is used for MOS calculations of an IV throughout this thesis (cf. Figure 3.2).

$$
(3.6,3.7) \Rightarrow e_{i}(x)=h \cdot\left(1-e^{-\left(\frac{x}{x_{0}}\right)^{m} \cdot \ln \left(\frac{h}{h-e_{0}+\mu}\right)}\right)+\mu
$$

\subsubsection{AV QoE Function}

Following the principles of Equation 3.6, the function $[0,1] \stackrel{e_{a}}{\rightarrow}[\mu, M], m \in$ $[0, \infty)$ (cf. Equation 3.9) is defined to correspond to the QoE of an AV (e.g., price). The $e_{a}$ function is illustrated in Figure 3.3. Similarly to $e_{i}$, values $x$ are unity-based normalized to enable multiple variables plotting in one graph. Furthermore, $e_{j}$ behaves also like a step function for large values of the influence factor $m$, like an exponential function for $m=1$, and like a constant function for $m \ll 1$. Finally, for $m \in(1,3] e_{a}$ also changes in a smooth way.

$$
e_{a}(x):=h \cdot e^{-\lambda \cdot x^{m}}+\mu
$$

In the $\mathrm{AV}$ case the $\mathrm{eV}^{2} x_{\circ}\left(0<x_{\circ}<1\right)$ results in an eMOS $e_{\circ}(\mu<$ $\left.e_{0}<M\right)$. Thus, $\lambda$ is presented in Equation 3.10, with the formal proof be 
in the Appendix A.1.

$$
\begin{aligned}
& e_{a}\left(x_{\circ}\right):=e_{\circ} \\
& \vdots \quad(\text { formally proven in the Appendix A.1) } \\
\Rightarrow & \lambda=x_{\circ}^{-m} \ln \left(\frac{h}{e_{\circ}-\mu}\right)
\end{aligned}
$$

Replacing $\lambda$ above in Equation 3.9 results Equation 3.11, which is the QoE function that is used for MOS calculations of a AV (cf. Figure 3.3).

$$
(3.9,3.10) \Rightarrow e_{a}(x)=h \cdot e^{-\left(\frac{x}{x_{0}}\right)^{m} \cdot \ln \left(\frac{h}{e_{0}-\mu}\right)}+\mu
$$

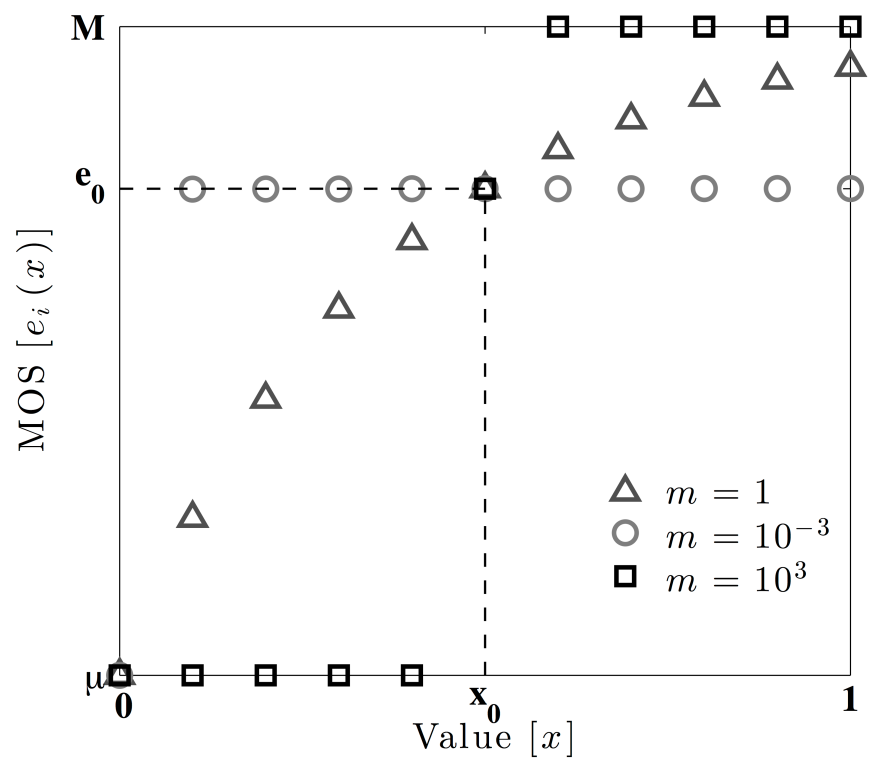

Figure 3.2: Plot for Different $m$ Values of Equation 3.8 


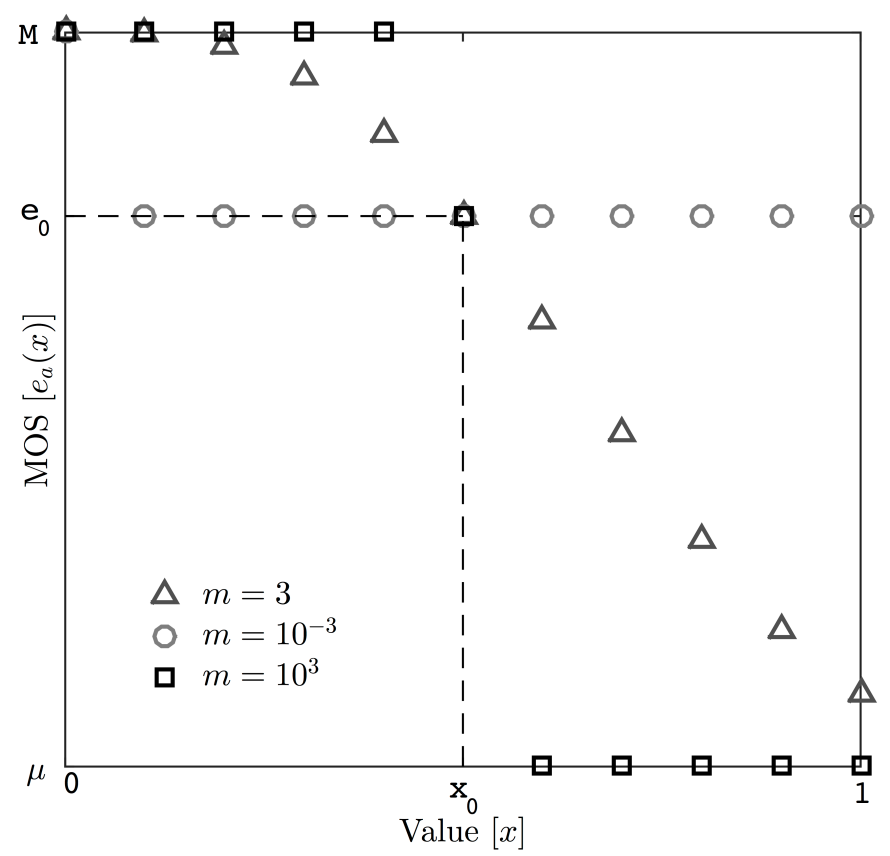

Figure 3.3: Plot for Different $m$ Values of Equation 3.11

\subsection{INFLUENCE FaCtors}

$\mathrm{AQX}$ assumes that equal fluctuations of variables might have different influence in QoE under certain circumstances. This assumption models end-users faster response in dissatisfaction-related incidents vs. end-users slower response to satisfaction-related incidents. Furthermore, selecting different influence factors $\mathrm{m}^{-}$and $\mathrm{m}^{+}$for a value of a variable above and below $x_{\circ}$ represents the flexibility of diverse end-user's preferences on different scenarios. To illustrate that, increasing the price $a>0$ of a product to $a^{\prime}=a+\xi$ for $\xi>0$ can effect differently end-user's QoE than decreasing the price of the same product to $a^{\prime \prime}=a-\xi$. Thus, Equation 3.12 shows that the influence factor $m$ is not necessarily constant. The influence factors approach adds a lot of flexibility in modeling of QoE, as well as a lot of sensitivity, due to influence factors dependance to the assumption of 
the impact that a variable's fluctuation has on QoE. Finally, since QoE is not a continuous function and thus not differentiable, the influence factors approach has been selected over the derivative-like concept.

$$
m:= \begin{cases}m^{-}>0 & \text { for } x<x_{\circ} \\ 0 & \text { for } x=x_{\circ} \\ m^{+}>0 & \text { for } x>x_{\circ}\end{cases}
$$

\subsubsection{Influence Factors Calculation}

Equation 3.13 presents the output of the calculation of $m^{+}$if $x=x_{0}+\delta<1$ for $\delta>$ o when the MOS of an IV is needed. Figure 3.4 shows that $e(x)=$ $e_{0}+\mathcal{E}<M$ for $\varepsilon>0$. Thus, the appropriate influence factor can be selected, if the impact of a specific value change on $\mathrm{QoE}$ is known. For instance, the

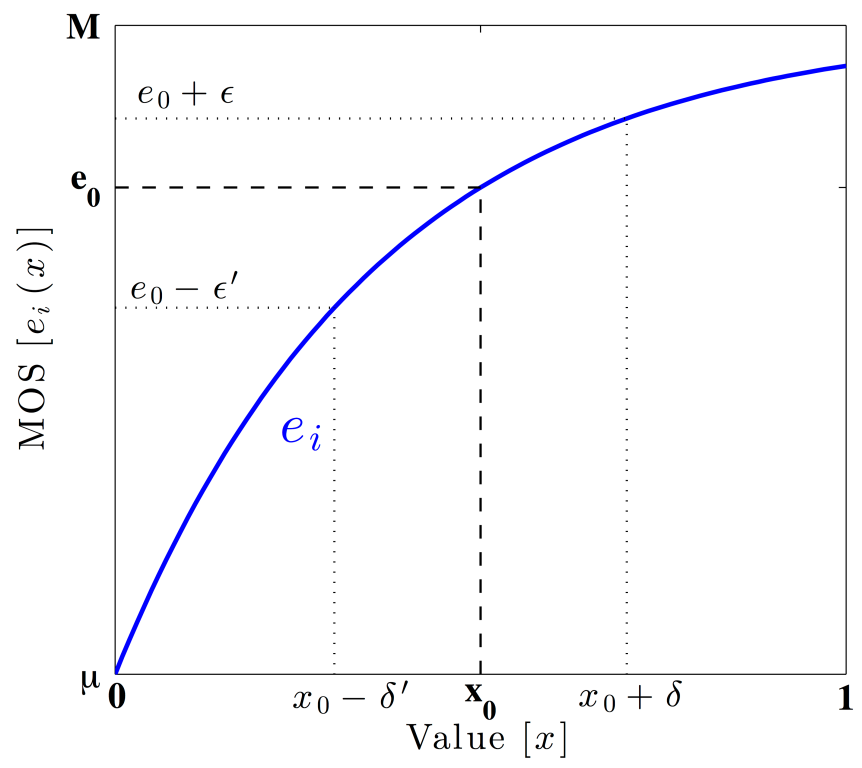

Figure 3.4: MOS Change for IV 
quality of a video streaming session will drop from High Definition (HD) to SD if the bandwidth will drop from from 3 Mbps to 1.5 Mbps [67]. QoE decrement will follow accordingly.

$$
\begin{aligned}
& e_{i}\left(x_{\circ}+\delta\right)=e_{\circ}+\varepsilon \\
& \Leftrightarrow h \cdot\left(1-e^{-\lambda \cdot\left(x_{0}+\delta\right)^{m^{+}}}\right)+\mu=e_{0}+\varepsilon \\
& \Leftrightarrow 1-e^{-\lambda \cdot\left(x_{\circ}+\delta\right)^{m^{+}}}=\frac{e_{\mathrm{o}}-\mu+\varepsilon}{h} \\
& \Leftrightarrow e^{-\lambda \cdot\left(x_{0}+\delta\right)^{m^{+}}}=\frac{h-e_{0}+\mu-\varepsilon}{h} \\
& \Leftrightarrow e^{-\left(\frac{x_{0}+\delta}{x_{0}}\right)^{m^{+}} \cdot \ln \left(\frac{h}{h-e_{0}+\mu}\right)}=\frac{h-e_{0}+\mu-\varepsilon}{h} \\
& \Leftrightarrow e^{\ln \left(\frac{h-e_{0}+\mu}{h}\right)^{\left(\frac{x_{0}+\delta}{x_{0}}\right)^{m^{+}}}}=\frac{M-e_{0}-\varepsilon}{h} \\
& \Leftrightarrow\left(\frac{h-e_{\circ}+\mu}{h}\right)^{\left(\frac{x_{0}+\delta}{x_{\circ}}\right)^{m^{+}}}=\frac{M-e_{\circ}-\varepsilon}{h} \\
& \Leftrightarrow\left(\frac{M-e_{0}}{h}\right)^{\left(\frac{x_{0}+\delta}{x_{\circ}}\right)^{m^{+}}}=\frac{M-e_{\circ}-\varepsilon}{h} \\
& \Leftrightarrow\left(\frac{x_{\circ}+\delta}{x_{\circ}}\right)^{m^{+}}=\log _{\left(\frac{M-e_{0}}{h}\right)}\left(\frac{M-e_{\circ}-\varepsilon}{h}\right) \\
& \Leftrightarrow m^{+}=\log _{\left(\frac{x_{0}+\delta}{x_{0}}\right)}\left[\log _{\left(\frac{M-e_{0}}{h}\right)}\left(\frac{M-e_{\circ}-\varepsilon}{h}\right)\right]
\end{aligned}
$$
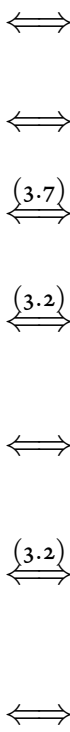

The base and the argument of each logarithm in Equation 3.13 is positive and different than 1 , because (a) $\circ<\frac{x_{0}+\delta}{x_{0}} \neq 1$, (b) $\circ<\frac{M-e_{0}}{h}<1$, and (c) $\circ<\frac{M-e_{0}-\varepsilon}{h}<1$. Thus, Equation 3.13 after changing the logarithm to the natural logarithm is rewritten below (any logarithm with a positive base different than 1 , such as the natural logarithm, can be selected for the logarithm base change to ease calculations). A careful selection of logarithms' base can simplify calculations e.g., $\log _{\alpha} \alpha=1$ where $\circ<a \neq 1$. Similarly to the previous case, when $x=x_{0}-\delta^{\prime}>$ o for $\delta^{\prime}>$ o and $e_{0}-\varepsilon^{\prime}>\mu$ for 
$\mathcal{E}^{\prime}>$ o (cf. Figure 3.4), $m^{-}$is seen in Equation 3.15 below, with the formal proof be in the Appendix A.1.

$$
\begin{gathered}
(3.13) \Rightarrow m^{+}=\frac{\ln \left(\frac{\ln \frac{M-e_{0}-\varepsilon}{h}}{\ln \frac{M-e_{0}}{h}}\right)}{\ln \frac{x_{0}+\delta}{x_{\circ}}} \\
m^{-}=\frac{\ln \left(\frac{\ln \frac{M-e_{o}+\varepsilon^{\prime}}{h}}{\ln \frac{M-e_{0}}{h}}\right)}{\ln \frac{x_{\circ}-\delta^{\prime}}{x_{\circ}}}
\end{gathered}
$$

Equation 3.13 and Equation 3.15 present the $m^{+}$and $m^{-}$calculation when $x=x_{\circ}+\delta$ and $x=x_{\circ}-\delta^{\prime}$ respectively. However, if the influence factor of an IV $m_{i, x}$ between any pair of $\left(x_{1}, x_{2}\right)$ and $\left(e_{i}\left(x_{1}\right), e_{i}\left(x_{2}\right)\right)=$ $\left(e_{i, 1}, e_{i, 2}\right)$ for $\circ<x_{1}<x_{2}<1$ is needed, Equation 3.18 can be used. To calculate $m_{i, x}$, a relation between $x_{1}, x_{2}$, and $e_{i, 1}, e_{i, 2}$ is needed. Thus the following calculations are done:

$$
e_{i}\left(x_{1}\right)=e_{i, 1} \stackrel{(3.6)}{\Longleftrightarrow}-\lambda \cdot x_{1}{ }^{m}=\ln \frac{h-e_{i, 1}+\mu}{h}
$$

Similarly for $x=x_{2}$.

$$
e_{i}\left(x_{2}\right)=e_{i, 2} \stackrel{(3.6)}{\Longleftrightarrow}-\lambda \cdot x_{2}{ }^{m}=\ln \frac{h-e_{i, 2}+\mu}{h}
$$

Dividing Equation 3.16 by Equation 3.17, and since $1 \neq x_{1} / x_{2} \neq 0$ :

$$
\begin{aligned}
\frac{(3.16)}{(3.17)} & \Leftrightarrow\left(\frac{x_{1}}{x_{2}}\right)^{m_{i, x}}=\frac{\ln \frac{h-e_{i, 1}+\mu}{h}}{\ln \frac{h-e_{i, 2}+\mu}{h}} \\
& \Leftrightarrow m_{i, x}=\log _{\left(\frac{x_{1}}{x_{2}}\right)\left[\frac{\ln \frac{h-e_{i, 1}+\mu}{h}}{\ln \frac{h-e_{i, 2}+\mu}{h}}\right]} \\
& \Leftrightarrow m_{i, x}=\frac{\ln \left(\frac{\ln \frac{M-e_{i, 1}}{h}}{\ln \frac{M-e_{i, 2}}{h}}\right)}{\ln \frac{x_{1}}{x_{2}}}
\end{aligned}
$$




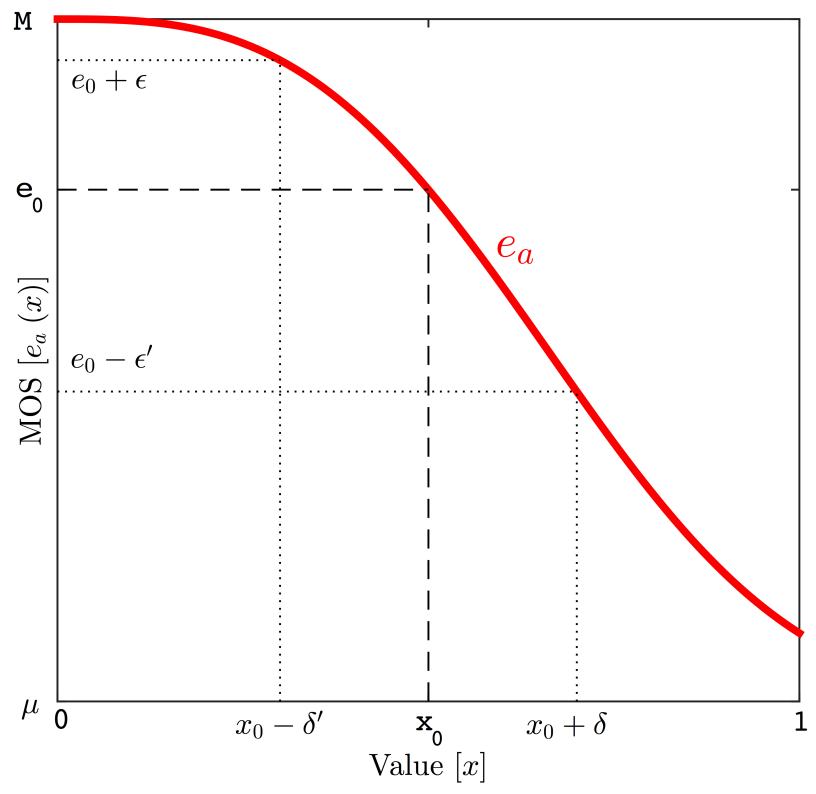

Figure 3.5: MOS Change for AV

\subsubsection{AV Influence Factors Calcultation}

To calculate the influence factors of $\mathrm{AV}$, symmetrical steps as in the influence factors calculation of IV are taken when considering AV (cf. Figure 3.5). Furthermore, symmetrical assumptions are done concerning $\delta, \delta^{\prime}$, $x_{\circ}+\delta, x_{\circ}-\delta^{\prime}$ and $\varepsilon, \varepsilon^{\prime}, e_{0}+\varepsilon, e_{\circ}-\varepsilon^{\prime}$, so that the flexibility on logarithms' base selection applies also in AV influence factors. Therefore, Equation 3.19, Equation 3.20, and Equation 3.21 are obtained.

For $x=x_{\circ}+\delta<1$, where $\delta>0$ and $e_{0}-\varepsilon^{\prime}>\mu$, where $\varepsilon^{\prime}>$ o (cf. Figure 3.5), $\mathrm{m}^{+}$is shown in Equation 3.19 below, which is formally proven in the Appendix A.1.

$$
\begin{aligned}
& e_{a}\left(x_{\circ}+\delta\right)=e_{\circ}-\varepsilon^{\prime} \\
& \vdots \quad(\text { formally proven in the Appendix A.1) }
\end{aligned}
$$




$$
\Leftrightarrow m^{+}=\log _{\left(\frac{x_{0}+\delta}{x_{\mathrm{o}}}\right)}\left[\log _{\left(\frac{e_{0}-\mu}{h}\right)}\left(\frac{e_{\mathrm{o}}-\mu-\mathcal{\varepsilon}^{\prime}}{h}\right)\right]
$$

Changing all the logarithms in Equation 3.19 to the natural logarithms is allowed since all the bases and arguments are positive and not equal to 1 . Thus, $m^{+}$is seen in Equation 3.20. Similar to the IV influence factors any logarithm with a positive base different than 1 can be selected for the logarithm base change to ease calculations.

$$
(3.19) \Rightarrow m^{+}=\frac{\ln \left(\frac{\ln \frac{e_{0}-\mu-\varepsilon^{\prime}}{h}}{\ln \frac{e_{0}-\mu}{h}}\right)}{\ln \frac{x_{0}+\delta}{x_{0}}}
$$

For $x=x_{\circ}-\delta^{\prime}>$ o where $\delta^{\prime}>$ o and $e_{\circ}+\varepsilon<M$ for $\varepsilon>0$ (cf. Figure 3.5), $\mathrm{m}^{-}$is seen in Equation 3.21 below, which is formally proven in the Appendix A.1.

$$
e_{a}\left(x_{\circ}-\delta^{\prime}\right)=e_{\circ}+\varepsilon
$$

: (formally proven in the Appendix A.1)

$$
\Leftrightarrow m^{-}=\frac{\ln \left(\frac{\ln \frac{e_{0}-\mu+\varepsilon}{h}}{\ln \frac{e_{0}-\mu}{h}}\right)}{\ln \frac{x_{0}-\delta^{\prime}}{x_{0}}}
$$

Similar to Equation 3.18, any calculation of $m=m_{a, x}$ for AV variables can be done for any pair of $\left(x_{1}, x_{2}\right)$ and $\left(e_{a}\left(x_{1}\right), e_{a}\left(x_{2}\right)\right)=\left(e_{a, 1}, e_{a, 2}\right)$ for $\mathrm{o} \geq x_{1}<x_{2} \leq 1$ (cf. Equation 3.24). To calculate $m_{a, x}$, a relation between $x_{1}, x_{2}$, and $e_{a, 1}, e_{a, 2}$ is needed. Thus the following calculations are done:

$$
e_{a}\left(x_{1}\right)=e_{a, 1} \stackrel{(3.9)}{\Longleftrightarrow}-\lambda \cdot x_{1}^{m}=\ln \frac{e_{a, 1}-\mu}{h}
$$

Similarly for $x=x_{2}$.

$$
e_{a}\left(x_{2}\right)=e_{a, 2} \stackrel{(3.9)}{\Longleftrightarrow}-\lambda \cdot x_{2}{ }^{m}=\ln \frac{e_{a, 2}-\mu}{h}
$$


Dividing Equation 3.22 by Equation 3.23, and since $1 \neq x_{1} / x_{2} \neq 0$ :

$$
\frac{(3.22)}{(3.23)} \stackrel{\text { Appendix A.1 }}{\Longrightarrow} m_{a, x}=\frac{\ln \left(\frac{\ln \frac{e_{a, 1}-\mu}{h}}{\ln \frac{e_{a, 2}-\mu}{h}}\right)}{\ln \frac{x_{1}}{x_{2}}}
$$

\subsection{Multi-variable QoE Function}

Each service's unique characteristics, define a unique affect of QoE when some variable(s) are changing. Thus, combining a set $X=$ $\left\{x_{1}, \ldots, x_{k}, \ldots, x_{N}\right\}$ of $N \in \mathbb{N}^{+}$diverse variables values $x_{k}$, to calculate a generic MOS, demands weights $w_{k}$ for each variable, since their importance might be different for different services. Equation 3.25 defines the generic MOS function $E(X)$. Weights $w_{k} \in \mathbb{R}^{+}$reflect the contribution of all variables. As a starting point the selection of $w_{k}=1$ is made, since all variables are considered to equally contribute in QoE. However, $w_{k}$ is an additional degree of freedom that considers the diverse importance of each variable. Thus, $w_{k}$ is used to calibrate $\mathrm{AQX}$. For this purpose input from end-users can be used to extract those values of $w_{k}$ that reflect better QoE as reported from end-users.

Figure 3.6 illustrates the generic MOS function $E(X)$ of a hypothetical service where one $\operatorname{IV}\left(x_{1}\right)$ and one $\operatorname{AV}\left(x_{2}\right)\left(X=\left\{x_{1}, x_{2}\right\}\right)$ with an influence factor $m=1$ and $m=3, \forall x_{1}, x_{2}$ respectively, affect the QoE. In this example the contribution weight of both parameters is selected to be $50 \%$ to show what is the QoE effect of an equal percent fluctuation of each parameter. The white area on the graph marks all the possible pairs of both variables values which result in an $\operatorname{eMOS} E\left(X_{\circ}=\left\{x_{1,0}, x_{2,0}\right\}\right)=E_{0}$.

$$
E(X):=\mu+h \cdot \prod_{k=1}^{N}\left[\frac{e_{(i \vee a)}\left(x_{k}\right)-\mu}{h}\right]^{w_{k}}
$$

The generic MOS in Equation 3.25 is chosen to be a weighted product of all variable-specific MOS' instead of a weighted sum. The reason for that 


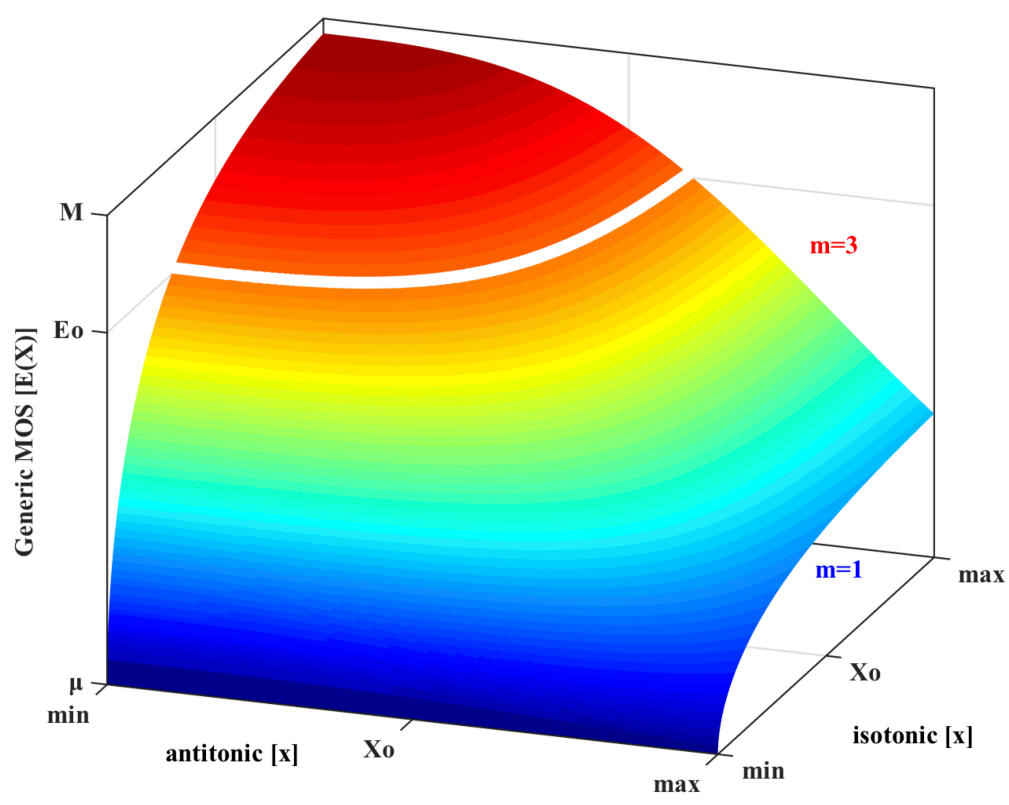

Figure 3.6: Generic MOS Evolution for Equally Participating IV $(m=1)$ and $\operatorname{AV}(m=3)$

is to ensure that if one variable's MOS is very low, and cannot be compensated by an improvement of other variables, the generic MOS will reflect it. In case of a weighted sum of each variable's MOS the generic MOS under specific circumstances can still be equal to the eMOS $E_{\circ}$ even if the MOS of a specific variable is the minimum one $\mu$.

To illustrate that an additive QoE model could in some cases not capture the negative effect of an outperforming variable affecting $\mathrm{QoE}$, assume a MvNO is offering a flat rate mobile data plan, and two variables (bandwidth (IV) and price (AV)) affecting equally the QoE of end-users. This is a realistic scenario since an end-user when selection such a service across different MvNOs with similar network coverage, the standard case for urban areas, can compare only those two variables. Assume that due to some technical problems, such as a link failure, the $\mathrm{MvNO}$ is unable to provide the 
service; considering only the bandwidth the MOS is minimum $(\mu)$ since there is no data connectivity. The MvNO decides not to charge customers for the service during the non-functional period; considering only the price the MOS is maximum $(M)$ since there is no cost for the service. If the generic MOS would be an equal weighted sum of the respective MOS', then $E=M / 2+\mu / 2$. For $M=5, \mu=1$, and $E_{\circ}=3$ the generic MOS would be $E=3=E_{\circ}$. However, the overall end-users' QoE despite the fact no payment is needed should be lower than the eMOS because no service is provided. Thus, the low credibility of the MvNO should also be reflected in the generic MOS. Equation 3.25 states the necessity of an acceptable level for each variable affecting $\mathrm{QoE}$. Thus, in the scenario mentioned above the generic MOS using Equation 3.25 would be $E=1+0=1$, which means that there is no price that a MvNO can offer to maintain end-users' QoE concerning the mobile data service if the service is not available. The latest result illustrates that the availability of a service, which in this scenario is encapsulated in the bandwidth variable, is an important parameter. Thus, the end-users' dissatisfaction and the MvNO's credibility decrement is reflected by the generic MOS for the service unavailability scenario described above.

\subsection{ITU-T MOS-Compliant QOE Functions}

The ITU-T has defined in recommendations P.80o [73], P.80o.1 [77], and P.805 [78] a five-point scale that represents $\mathrm{QoE}$ of end-users. The ITU-T MOS scale is summarized in Table 2.1. In the AQX model, the eMOS $e_{0}$, is selected to be equal to the ITU-T numerical representation of "Good" $\mathrm{QoE}$, when the value of a variable $x$ is equal to the $\mathrm{eV}^{2} x_{0}$. Thus, Equation (3.26) illustrates the ITU-T MOS-compliant maximum $M$, expected $e_{0}$, and minimum $\mu$ MOS values.

$$
\left.\begin{array}{r}
M:=5 \\
\mu:=1
\end{array}\right\} \stackrel{(3.2)}{\longrightarrow} h=4 \text { and } e_{0}:=4
$$

Given this input, the ITU-T MOS-compliant equation for IV and the influence factor $m$ equations, as presented in Section 3.2 and Section 3.3 re- 
spectively, can be seen in Equation 3.27, Equation 3.29, and Equation 3.28. Similarly for AV, the same results are formalized in Equation 3.30 , Equation 3.32, and Equation 3.31. Those equations can be used to express the QoE in the ITU-T standardize five point scale e.g. when considering telephony services.

$$
\begin{gathered}
(3.8,3.26) \Rightarrow e_{i}(x)=4 \cdot\left(1-e^{-\left(\frac{x}{x_{0}}\right)^{m} \cdot \ln 4}\right)+1 \\
(3.14,3.27) \Rightarrow m^{+}=\frac{\ln \left(\frac{\ln \frac{1-\varepsilon}{4}}{-\ln 4}\right)}{\ln \frac{x_{0}+\delta}{x_{0}}} \\
(3.15,3.27) \Rightarrow m^{-}=\frac{\ln \left(\frac{\ln \frac{1+\varepsilon^{\prime}}{4}}{-\ln 4}\right)}{\ln \frac{x_{0}-\delta^{\prime}}{x_{0}}} \\
(3.11,3.26) \Rightarrow e_{a}(x)=4 \cdot e^{-\left(\frac{x}{x_{0}}\right)^{m} \cdot \ln 4 / 3}+1 \\
(3.20,3.30) \Rightarrow m^{+}=\frac{\ln \left(\frac{\ln \frac{3-\varepsilon^{\prime}}{4}}{\ln 3 / 4}\right)}{\ln \frac{x_{0}+\delta}{x_{0}}} \\
(3.21,3.30) \Rightarrow m^{-}=\frac{\ln \left(\frac{\ln \frac{3+\varepsilon}{4}}{\ln 3 / 4}\right)}{\ln \frac{x_{0}-\delta^{\prime}}{x_{0}}}
\end{gathered}
$$

Finally, Equation 3.33 generates the generic ITU-T MOS-compliant MOS $E(X)$ considering a set $X$ of IV' and AV' values. Such a method is used at $[138]$ to calculate the MOS of various services on mobile networks such as VoIP, video streaming, browsing, and random flow data streaming.

$$
(3.25,3.26) \Rightarrow E(X)=1+4 \cdot \prod_{k=1}^{N}\left[\frac{e_{(i \vee a)}\left(x_{k}\right)-1}{4}\right]^{w_{k}}
$$




\subsection{Concurrent Economic and Technical Parameters CONSIDERATION}

Influence factors model the percentage of MOS fluctuation from $e_{0}$ for a specific fluctuation of a variable's value from $x_{0}$. To calculate the influence factor one must know/estimate/assume/predict what will be the percentage of the increment (or decrement) of end-users satisfaction in terms of MOS, for a specific percentage of the increment (or decrement) of a variable's value, such as the price of a service. The scenario of QoE estimation of a voice call while considering technical variables, such as sound quality, and economic variables such as, Mobile Termination Rates (MTR), is presented briefly in Chapter 5. There it is shown how parameters of AQX model are selected when economic and technical variables are considered concurrently. The following steps are derived from the axioms of QoE modeling with AQX, when considering multiple and diverse variables, and a preview of the mobile voice call parameters, which will be presented in detail in Chapter 5 is found below:

- Identify all variables that affect $\mathrm{QoE}$ and can be influenced by the SP - Table 5.3

- Characterize those variables (IV, AV)

- Table 5.4

- Select the ideal/desired/expected/agreed $x_{\circ}$ value of each variable

— Table 5.5, Table 5.6

- Considering the service specifications select the best and the worst values of each variable

— Table 5.5, Table 5.6

- Identify the effect of each variable's variation (influence factors $m$ )

- Table 5.7

- Identify the importance of each variable (importance factors $w$ )

- Table 5.10 


\subsection{Chapter Summary}

AQX adopted the generic exponential QoE model ideas and proposes an axiomatic mathematical model that can be used to generate a MOS illustrating end-user QoE considering one or multiple and diverse variables, such as bandwidth, network access guarantees, or price. The generalized MOS estimation function proposed in AQX, is defining MOS as a weighted product of all variable-specific MOS' (referring to the MOS' of individual influence factors) rather than a weighted sum. The rationale behind this approach is that the model should effectively reflect the situation when one variable's MOS is very low and cannot be compensated by an improvement of other variables. The model requires a minimum and maximum satisfaction score in the positive numbers space and a value between those numbers to represents the end-user's satisfaction when each variable has the desired/expected value, such as the minimum required bandwidth needed to achieve HD video streaming quality. Furthermore, for each variable a positive number is needed showing the effect of the parameter's fluctuation in QoE. This influence factor can be calculated from those equations that are provided here. In case of more than one participating variable in the MOS calculation the importance of each variable needs to be specified. Additionally, AQX is aligned with the comprehensive framework for $\mathrm{QoE}$ and User Behavior (UB) modeling presented at [ 115$]$, since the reference value for each parameter can be specified as a parameter in AQX. E.g., maximum latency a specific end-user can tolerate at a given VoIP scenario.

Concluding, such a model presented here is very well suited (a) to calculate the MOS provided the value of measurable variables and (b) to estimate which actions are needed in case of a change, of one or more variable(s') value(s), to maintain the same end-user's satisfaction level if possible. Having such a method in place can save the time needed to perform a survey to observe end-user behavior. Also, the MOS generation mathematical model presented here, can be used to generate a comparison index on similar services for the end-user, across different service providers, such as CPs, with different variables and values. 
AQX is beneficial for end-users when comparing different services across multiple SPs. SPs can also estimate the end-user's QoE with such a mathematical model while considering (a) their available resources, (b) demands of the end-user, and (c) minimum service requirements. Furthermore, since this model can consider variables, such as the price of a service, SPs can maximize their revenues while maintaining their customer's QoE at a certain level. Thus, it is essential for SPs to create end-user and service profiles by calculating all parameters of this model. Such a calculation can be initiated through end-user's demands (e.g., maximum price for a service), and/or service's characteristics (e.g., maximum latency allowed on a VoIP session). 


\section{4 \\ AQX in VoIP}

UAlity-OF-Service is defined by application-specific values thresholds of technical variables such as latency, packet loss, and bandwidth. These values are well known for different technologies and services and they can be measured [78]. Furthermore, selected values of those variables are often used for marketing purposes, e.g., MvNOs and ISPs advertise "high bandwidth" or "high performance". However, QoS variables are not explicitly linked to the end-user's satisfaction. It is naive to conclude that end-users' QoE can be increased by adjusting one QoS variable, because the relationship between $\mathrm{QoS}$ variables and end-users' experience depends on the Type-of-Service (ToS). Large latency can serve as an example here, since latency has a higher negative effect on VoIP services than on video streaming [74].

This chapter focuses on applying AQX in VoIP to validate its usability and quantify its performance. The four step QoE formalization methodology in this chapter reads as follows: (1) Define an experimental setup allowing for the emulation of various network connection performance settings on latency, packet loss, and bandwidth; (2) perform test VoIP calls in pre-defined and controlled experimental setups and collect QoE-related feedback from end-users in terms of MOS [77]; (3) use the feedback col- 
lected to evaluate the AQX model results for different variables in this VoIP scenario by comparing AQX [140] when only one QoE-related variable is considered to the two QoE-predicting models, (i) the single-value exponential relationship connecting QoS parameters, called IQX hypothesis [50], and (ii) the multi-value E-model [76] of the ITU-T; and (4) evaluate the performance of AQX when multiple technical variables are considered.

In support of the accurately and timely measurement of QoE for VoIP services, the state-of-the-art Web Real-Time Communications (WebRTC) technology was used to newly design and develop a VoIP client that collects directly all necessary user's feedback concerning the perceived communication quality, in experimental VoIP calls, under different network conditions in various scenarios [122] [137]. Those varying network conditions are emulated by the network emulation framework WANem [130], which utilizes a real network. Therefore, three computers were attached to each other through a switch via LAN cables. Using such an experimental architecture guarantees a fully controlled network emulation that is not influenced by external traffic.

This experimental setup served for the collection of more than 500 data points and was used to show how accurate the previously mentioned QoEpredicting models [50][76] reflect these collected data points. In this experiment, it was shown that the AQX model is the most accurate model to capture QoE in given scenarios.

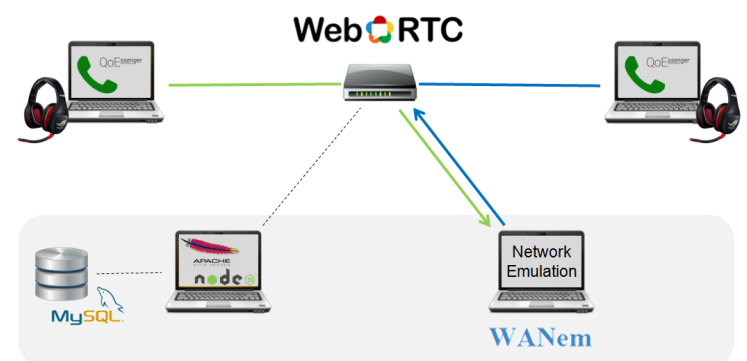

Figure 4.1: Experimental Setup 


\subsection{Experimental Setup}

The experimental setup is based on the WANem framework [130] for network emulation and a WebRTC messenger that was developed and used for the experiments of this thesis. Moreover the ITU recommendation P.80o [73] was considered for the experimental procedure.

\subsubsection{Hardware ANd Software Architecture}

A key element of the QoE evaluation architecture used in this thesis is a WebRTC messenger. WebRTC is World Wide Web Consortium $\left(\mathrm{W}_{3} \mathrm{C}\right) \mathrm{draft}$ standard for real-time communication between browsers [146]. The goal of WebRTC is plug-in-free low-cost communication in real-time between any browser. And with communication not only audio and video communication is meant, but also the direct exchange of data. So with the help of WebRTC it is possible to create a Peer-to-Peer $\left(\mathrm{P}_{2} \mathrm{P}\right)$ connection from browser to browser and send audio, video and data over it. This WebRTC approach has been chosen because it is the new trend in the field of the VoIP communication, it is open source and there is no software necessary other than a browser on the client side [141].

Additionally, the WANem [130] framework for network emulation was used. This software, which is based on the Linux OS Knoppnix [88], is convincing because it makes use of the well-accepted open source network tool for Linux called NetEm [84][92]. Furthermore, WANem provides the possibility to build a UI on top of it, which facilitates the flexibility to create a control panel that meets with precision the demands of an QoE evaluation experiment, such as the easy setup of different scenarios with diverse latency, bandwidth, and packet loss settings to be tested, as well as the automated collection of the user ratings.

The architecture using WANem is illustrated in Figure 4.1. There are four computers connected in a local LAN through a switch. Two computers run the WebRTC messenger, one computer runs an Apache [18] web server, a node.js [83] signaling server as well as a MySQL [18] data base and the last computer runs the WANem tool that can emulate the network. The 
WANem works as follows: The routing table of the two computers that run the WebRTC messenger are modified in such a manner, that all the packets are routed to the other peer through the computer that runs WANem. This computer is responsible for the network emulation. E.g., if the packet loss is set to $50 \%$, the WANem computer will drop every second packet that is routed through. Such architecture with LAN cables and a switch is necessary to guarantee a controlled network environment without the interferences that happen in a Wireless Local Area Network (WLAN) network.

\subsubsection{Experimental Procedure}

The experimental procedure besides the hardware and software -related information, includes some important information concerning (a) the participants group (subjects) and (b) the procedure of the experiments. The subjects of the experimental procedure of this experiment were 34 volunteers at the University of Zürich and the High School of Willisau in Switzerland.

The volunteers were mainly computer science students between 20 and 25 years old. However, the overall age distribution range of the subjects was from 16 to 63 years old. A pair of two randomly selected subjects participated in several voice calls with different technical parameters. Each subject rated the quality of each call separately after the end of the call.

The goal of the overall experimental procedure was to affect as little as possible the QoE rating of each subject. Firstly, the number and the duration of the test calls defined carefully, so that the experiment about the human experience would not demand from a subject to actively participate for more than 30 minutes in voice calls. Otherwise, it was assumed that the subjects would become annoyed and/or bored and their answers would be influenced by emotions which would decrease the quality of the results. To avoid such situation, the total duration of each experimental session designed to not exceed one hour.

Having a fixed interview length influenced the decision concerning the number and the duration of the test calls. There is a trade-off between the number of measurements and the confidence of the results. If the test calls are longer, fewer experiments can be performed within a fixed time-frame. 
It was assumed that people are not able to have a free and balanced conversation of 45 second on their own since it does not seem to be enough time to develop a proper conversation, especially between strangers.

To avoid unbalanced conversations, the following method was used to support the conversation in the test calls: At the beginning of the experiment each participant got around 300 easy general knowledge questions [87] [104] and the subjects had to ask and answer them alternately. This approach led to a fluent and balanced conversation without distracting the subjects from their evaluation task.

The decision about the procedure of the interview was as follows:

- o-5 min Introduction, explanation of the experiment and rating system

- 5-25 min 16 Test Calls, around 45 seconds calling time + 15 seconds voting time each

- 25-30 min Question and Answers about the calling experience

\subsection{AQX PARAMETERS IN VOIP}

The following evaluation is based on the MOS of 34 subjects, which produced in total more than 500 end-user's opinion score ratings at an overall calling time of approximately 6 hours. $80 \%$ of these ratings were collected in a single variable scenario, where only one variable was adjusted. The remainder of these ratings were mixed variable scenarios, where multiple variables were adjusted simultaneously. The main focus of this experiment's QoE measurements was on the single variable scenarios, since AQX only demands knowledge of expected variable values and influence factors of individual variables to predict $\mathrm{Q}$ EE considering multiple variables. The primary goal of this experiment was to calibrate AQX for the VoIP scenario. Thus, the data were collected for this purpose. The secondary goal of this experiment was to validate the AQX prediction performance in the multiple variables scenario. It was assumed that less but equally distributed set 
of data points would be sufficient to reveal the potential inaccuracy of the AQX model.

Although the AQX model can be calibrated for diverse technical and non-technical variables, in this experiment the comparison of $A Q X$ against other state of the art models is attempted. Thus, the focus is on four technical network-related variables that affect QoE in VoIP: (1) latency, (2) packet loss, and (3) bandwidth. The AQX model needs for every technical variable an expected variable value $x_{\circ}$ [140] [138].

The WebRTC technology that was used in this experiment is relatively young and rich literature, that can be used to find possible expected variable values, does not yet exist. Thus, to select $x_{\circ}$ values several references were used $[74][75][30][107][148]$. Forthermore, the minimum and maximum values $\left(x_{\min }, x_{\max }\right)$ for each variable have been selected under the following assumptions since no similar study requiring such values could be found in literature by the time that the work of this thesis concluded:

- In best case there is no latency, or packet loss $\left(x_{\min }=\mathrm{o}\right.$ for all variables)

- In the worst case the maximum latency in VoIP is equal to approximately the one way delay of satellite communication and any delay above this is assumed to be similarly undesirable $\left(x_{\max }=1600\right)$

- Concerning packet loss, any packet loss above $40 \%$ is assumed to be equally undesirable $\left(x_{\max }=40\right)$

- Concerning the bandwidth any value approximately below $1 / 3$ of the required bandwidth according to the VoIP protocol $\left(x_{\circ}=65\right)$ is assumed to be equally undesirable $\left(x_{\min }=20\right)$

- Any bandwidth value approximately twice as $x_{\circ}$ will not affect a call's QoE further in a positive way $\left(x_{\max }=125\right)$

Finally, each variable must be characterized as AV, or IV. Thus, each variable's AV/IV characterization, the $x_{0}, x_{\text {min }}$, and $x_{\text {max }}$ values and references as of Table 4.1 were used for this evaluation 
Table 4.1: $x_{0}, x_{\min }, x_{\max }$ and References Used for the Evaluation

\begin{tabular}{|c|c|c|c|c|c|}
\hline $\begin{array}{c}\text { AV/IV } \\
\text { Behavior }\end{array}$ & $\begin{array}{c}\text { Variable } \\
{[\text { unit }]}\end{array}$ & $x_{\circ}$ & $x_{\min }$ & $x_{\max }$ & Reference \\
\hline \hline $\mathrm{AV}$ & $\begin{array}{c}\text { Latency } \\
\text { (two way) }[\mathrm{ms}]\end{array}$ & 300 & $\circ$ & 1600 & {$[74][75]$ ITU } \\
\hline $\mathrm{AV}$ & $\begin{array}{c}\text { Packet loss } \\
{[\%]}\end{array}$ & 5 & 0 & 40 & $\begin{array}{c}{[107] \text { Opus }} \\
\text { documentation }\end{array}$ \\
\hline $\mathrm{IV}$ & $\begin{array}{c}\text { Bandwidth } \\
{[\mathrm{kbit} / \mathrm{s}]}\end{array}$ & 65 & 20 & 125 & $\begin{array}{c}{[148] \text { WebRTC }} \\
\text { official blog }\end{array}$ \\
\hline \hline
\end{tabular}

\subsubsection{Single Variables}

AQX generic QoE function demands knowledge of the QoE function of each variable affecting QoE. Furthermore, the IQX Hypothesis can consider simultaneously only one variable affecting QoE. Thus, the main focus of the experiments was (a) to select AQX parameters for each variable, such as the expected value and the influence factor of a variable, based on literature, or intuitive assumptions when references were not available, and (b) to compare AQX performance against IQX and E-model for those variables. Here those results of each variable scenario are presented. To demonstrate with an example, in a scenario with $5 \%$ packet loss, the latency was set to zero milliseconds (o ms) and the bandwidth was unlimited so that only packet-loss affects QoE.

Once $x_{0}, x_{\text {min }}$, and $x_{\max }$ are identified, to calculate influence factors $\mathrm{m}^{-}$ and $m^{-}$using Equations 3.283 .29 and Equations 3.313 .32 respectively, an assumption (if no available reference exist) of the MOS fluctuation is needed. Thus, the question to be answered reads: "If MOS $=4$ for $x=x_{0}$, what the MOS will be for $x=x_{\max }$, and $x=x_{\text {min }}$ ?". To answer this question for latency, packet loss, and bandwidth in VoIP the following assumptions are made:

- Latency $1600 \mathrm{~ms}$ will result a poor communication $(\mathrm{MOS}=2)$ due to large waiting time. Thus, (3.31) $\Rightarrow$ $m^{+}=\ln (\ln 3-2 / 4 / \ln 3 / 4) / \ln 300+1300 / 300=0.94$ 
- Latency $<<300 \mathrm{~ms}$ (e.g., $1 \mathrm{~ms}$ ) will not increase significantly QoE because the end-user cannot sense such small delays (e.g., maximum MOS=4.5). Thus, $(3.32) \Rightarrow m^{-}=\ln (\ln 3+0.5 / 4 / \ln 3 / 4) / \ln 300-299 / 300=$ 0.13

- Packet loss $40 \%$ will result a poor communication $(\mathrm{MOS}=2)$ due to many interruptions. Thus, $(3.31) \Rightarrow m^{+}=\ln (\ln 3-2 / 4 / \ln 3 / 4) / \ln 5+35 / 35=$ 0.76

- Packet loss $<<5 \%$ (e.g.,o.01\%) will not increase significantly QoE because the protocol is able to tolerate up to $5 \%$ packet loss (e.g., maximum MOS=4.5). Thus, (3.32) $\Rightarrow$ $m^{-}=\ln (\ln 3+0.5 / 4 / \ln 3 / 4) / \ln 5-4.99 / 5=0.12$

- Doubling bandwidth will not improve significantly QoE because the protocol is designed to perform well even with (65 kbit/s). Thus, maximum MOS $=4.5$ is expected even for such a generous bandwidth update. This results to: $(3.28) \Rightarrow m^{+}=\ln (\ln 1-0.5 / 4 /-\ln 4) / \ln 65+60 / 65=$ 0.62

- Significant decrement of bandwidth (e.g., 20 instead of $65 \mathrm{kbit} / \mathrm{s}$ ) will decrease significantly QoE because the protocol expects a minimum bandwidth to perform reliably. Thus, bad quality (e.g., MOS $\approx 1$ ) is expected. This results to: (3.29) $\Rightarrow$ $m^{-}=\ln (\ln 1+2.99 / 4 /-\ln 4) / \ln 65-45 / 65=5.36$

Table 4.2 summarizes influence factors $(m)$ values calculated from Equation 3.28, Equation 3.29 for the IV variable (Bandwidth), and Equation 3.31, Equation 3.32 for AVs (Latency, Packet loss).

Table 4.2: Influence Factors Results of the Single Variable Scenarios

\begin{tabular}{|l||c|c|c|}
\hline & Latency & Packet loss & Bandwidth \\
\hline \hline$m^{+}$ & 0.94 & 0.76 & 0.62 \\
\hline$m^{-}$ & 0.13 & 0.12 & 5.36 \\
\hline \hline
\end{tabular}




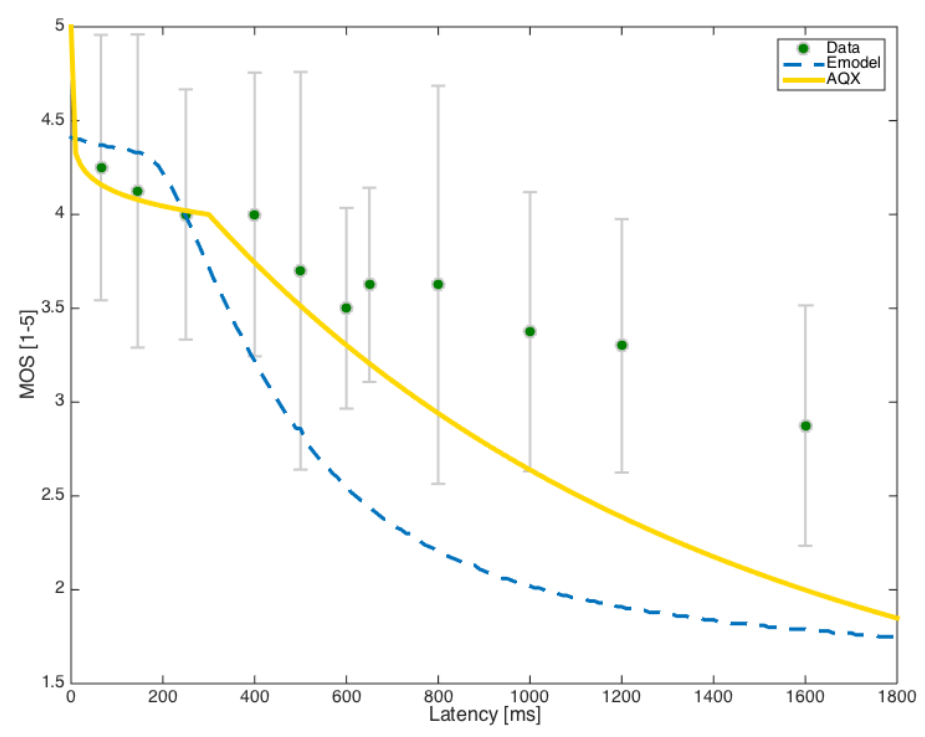

Figure 4.2: $A Q X$ vs Emodel (Latency)

The resulting graphs of the AQX model were also compared to the other two QoE-predicting models during this experiment. Figure 4.2 shows two plots which show the comparison between QoE results of the AQX and the ITU-T-standardised E-model (dashed line) as a function of latency. IQX hypothesis is missing in Figure $\mathbf{4 . 2}$ because there is not an equation proposed by IQX modeling MOS and latency. Thus, there are no IQX data available for latency. The error bars in the plot represent the standard deviation of the collected data.

It is noticeable in Figure 4.2 that the E-Model proposes lower MOS values than the AQX model most of the time. Where both models do not appear to be accurate enough with the collected data, AQX appears to be approach better collected data. The reason for such high values in the data set could be the following. Latency is a variable that when is high is not directly annoying, like bad audio quality is. Latency gets more annoying the longer and the faster a conversation becomes. High latency might not be 
very disturbing in a short conversation with small talk characteristics. The conversations of these experiments had exactly these characteristics. Thus, it was assumed that the subjects did not report low MOS for high latency.

Since the collected MOS values seemed rather high, some extra experiments were performed with longer experimental calls in which only latency was tested. For these calls, three different conversational tasks proposed by the ITU-T were tested: (a) a travel office role play, (b) a random number verification task and (c) a contacts exchange task [78]. The results of these extra tests were unexpected. The subjects rated still high. For a test scenario with $1500 \mathrm{~ms}$ latency the MOS was still 3.17. Therefore, it is further assumed that not only the duration and the type of conversational task are responsible for the unexpected outcome. It is possible that a cultural phenomenon leads to such results. As stated in [78] MOS can vary due to cultural differences. Except four subjects, all of them spoke Swiss German which is a rather slow language and therefore latency probably disturbs less. This hypothesis is supported by a test call between a Russian and an Italian participant held in English which seemed to be faster and more interactive than most of the native Swiss German speakers' conversations, and in this experiment the latency was not tolerated. Although a proof of this phenomenon could not be found in [118], since the sample was not large enough it stays only a hypothesis in this experiment.

This experiment showed that E-model would be incapable to spot such behavior. However, AQX could predict the right MOS with high accuracy with another influence factor selection under an appropriate assumption. E.g, latency $1600 \mathrm{~ms}$ will not result a poor communication $(\mathrm{MOS}=3)$ due to

Table 4.3: Updated Influence Factors Results of the Single Variable Scenarios

\begin{tabular}{|l||c|c|c|}
\hline & Latency & Packet loss & Bandwidth \\
\hline \hline$m^{+}$ & 0.52 & 0.76 & 0.62 \\
\hline$m^{-}$ & 0.13 & 0.12 & 5.36 \\
\hline \hline
\end{tabular}




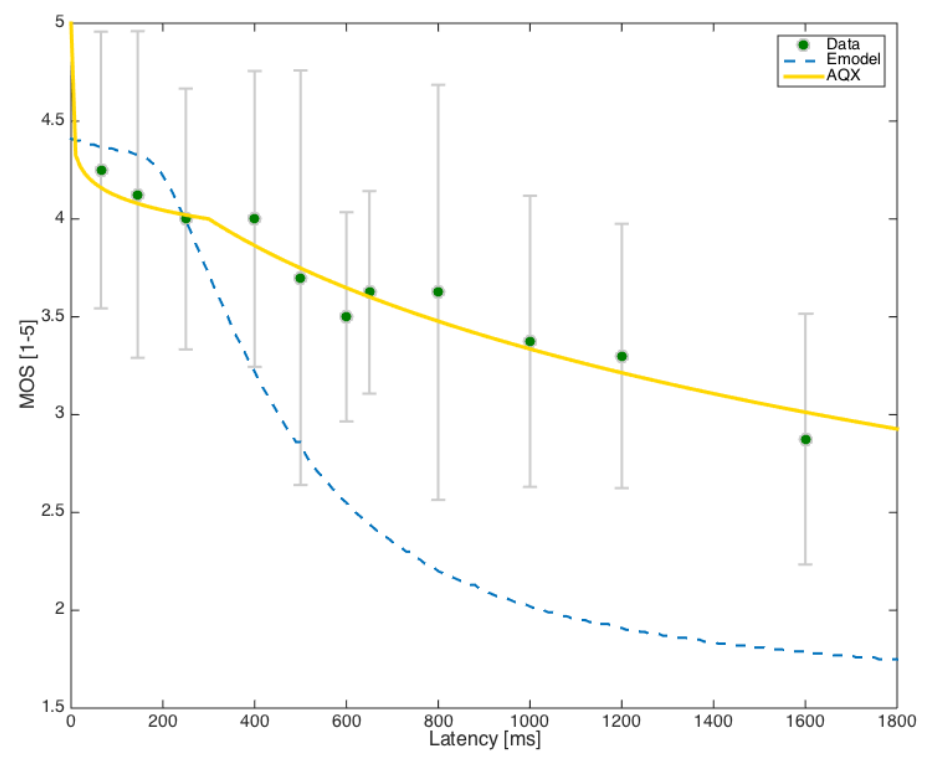

Figure 4.3: AQX vs Emodel (Latency - Updated $m^{+}$)

the high tolerance of Swiss German speakers participating in experiments of this thesis. Thus, $(3.31) \Rightarrow m^{+}=\ln (\ln 3-1 / 4 / \ln 3 / 4) / \ln 300+1300 / 300=0.52$.

Table 4.3 summarizes the updated influence factors $(m)$ values under the assumption that participants of experiments in this thesis could tolerate latency in such experiments. Figure 4.3 shows that AQX is adjustable and under the right assumption $m$ can selected appropriately so that AQX performs even better. Thus, it is shown that the E-model is not accurate in every VoIP scenario, since it was proven to be too pessimistic in respect to the negative effect of latency between slow-speaking subjects. The MOS depends on the service and the respective users. Therefore a model that allows flexible calibration, such as AQX, is needed to predict QoE accurately in diverse scenarios.

In Figure 4.4 the MOS as a function of the packet loss is illustrated and compared to the E-Model and the IQX hypothesis. The AQX model ap- 


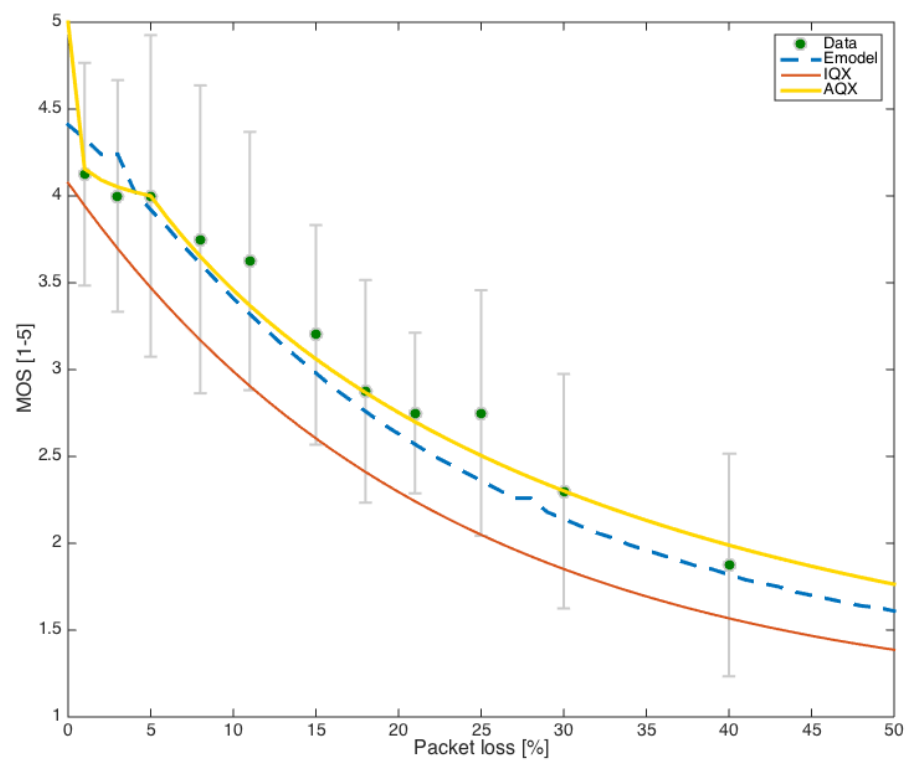

Figure 4.4: AQX vs Emodel and IQX (Packet Loss)

pears to capture QoE better than the E-model and the IQX hypothesis. In the IQX Hypothesis experiments [50] the Internet Low Bitrate Codec (iLBC) was used during the measurements. For the E-Model calculation tool [79] the G.711 Codec [81] is assumed, and the codec in those experiments was Opus [107]. The reason of having different codecs is that there was no control in codecs used in the related work and the use of the same codecs was not possible in WebRTC by the time of the experiments. However, Opus has advanced error correction mechanisms similar to the most advanced version of G.711 that is used in the E-Model and the one of the IQX hypothesis experiments. Therefore, it is possible to compare the results of different QoE models but for the same variables, which are presented in Figure 4.2 and Figure 4.4.

Finally, in Figure 4.5 the MOS as a function of the packet loss is illustrated. IQX hypothesis and Emodel are missing in Figure 4.5 because there 


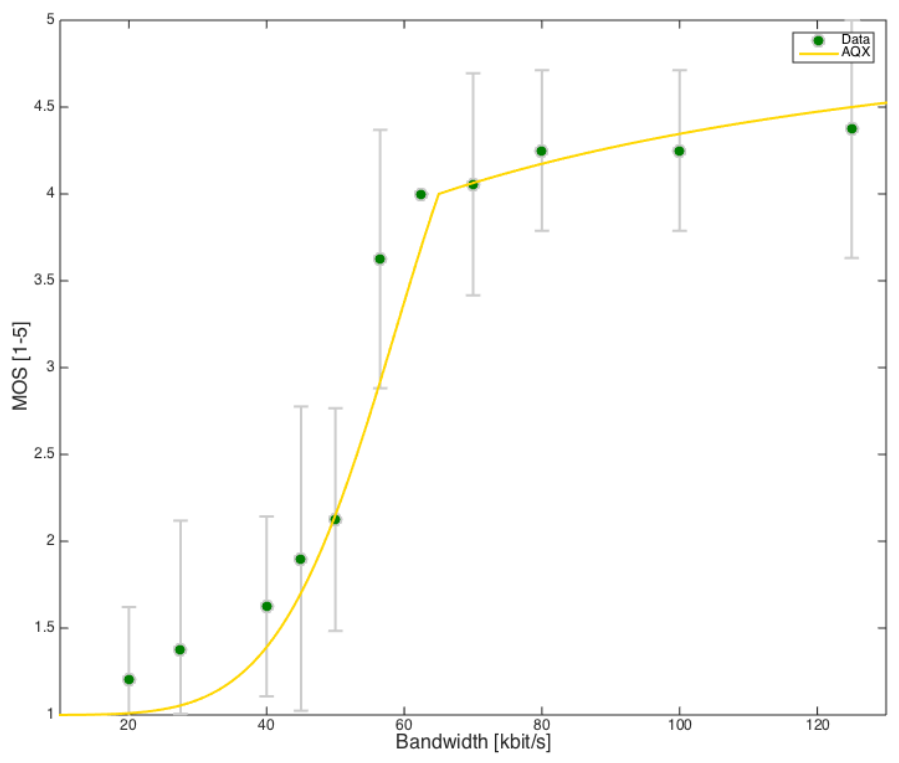

Figure 4.5: AQX MOS (Bandwidth)

is not an equation proposed by IQX nor Emodel modeling MOS and bandwidth only. Thus, there are only AQX data available for bandwidth.

\subsubsection{Multiple Variables Combination}

This part presents the AQX model for scenarios where multiple variables were tested. Since the main focus was on single variable tests, only five data points were collected for each of these mixed scenarios. However, the evaluation on those data points for different values of Latency (L), Packet Loss (PL), and Bandwidth (B), showed that AQX predicts accurately the QoE in these multiple variables VoIP scenarios.

In these experiments, 12 different multiple-variables-scenarios were tested and their results are summarized in Table 4.4. The first three columns indicate which variable values were tested. Column four contain the MOS 
Table 4.4: Collected MOS for Mixed Variables Compared to the Calculated MOS

\begin{tabular}{|c|c|c|c|c|}
\hline $\mathbf{L}[\mathbf{m s}]$ & PL $[\%]$ & $\mathbf{B}[\mathbf{k b p s}]$ & $\begin{array}{c}\text { Collected MOS } \\
\text { (std. dev.) }\end{array}$ & AQX \\
\hline \hline 600 & 10 & unlimited & $3.13(0.64)$ & 2.63 \\
\hline 500 & 7 & unlimited & $3.56(0.73)$ & 2.88 \\
\hline 500 & 10 & unlimited & $3.00(0.67)$ & 2.68 \\
\hline 500 & 10 & 60 & $3.25(0.46)$ & 2.00 \\
\hline 400 & 0 & 75 & $3.38(0.74)$ & 3.23 \\
\hline 400 & 7 & unlimited & $3.25(0.71)$ & 2.97 \\
\hline 400 & 20 & 75 & $2.50(0.93)$ & 1.98 \\
\hline 250 & 10 & 0 & $2.80(0.63)$ & 2.86 \\
\hline 0 & 7 & 64 & $3.88(0.64)$ & 2.99 \\
\hline 0 & 7 & 98 & $3.88(0.64)$ & 3.30 \\
\hline 0 & 10 & 60 & $3.25(0.46)$ & 2.46 \\
\hline 0 & 12 & 98 & $3.25(0.71)$ & 2.90 \\
\hline \hline
\end{tabular}

collected from the subjects in experiments as well as the standard deviations of these collected ratings. The last column shows the MOS calculated by the Equation 3.33 of the AQX model using the parameters determined by the single variable scenarios.

Comparing these results in Table 4.4 it has to be noted that Equation 3.33 creates promising results, since the differences between calculated and collected MOS are small. The mean of all MOS differences is 0.52 , which is small for an unadjusted calculation where all variables assumed to be equally important (all weights are equal to 1). Each variable's weight serves as another degree of freedom, allowing further calibration of AQX. However, there is not a sufficient amount of data points to make any significant statement in those experiments. Thus, it was not possible to use the additional degree of freedom that AQX allows for in these cases.

The result of this multiple variables scenario (latency and packet loss) is illustrated in Figure 4.6, where two variables are mixed. The ${ }_{3} \mathrm{D}$-curve is the calculated AQX model for the two variables and the large black bullets show the MOS collected. The size of these bullets has been chosen for vi- 


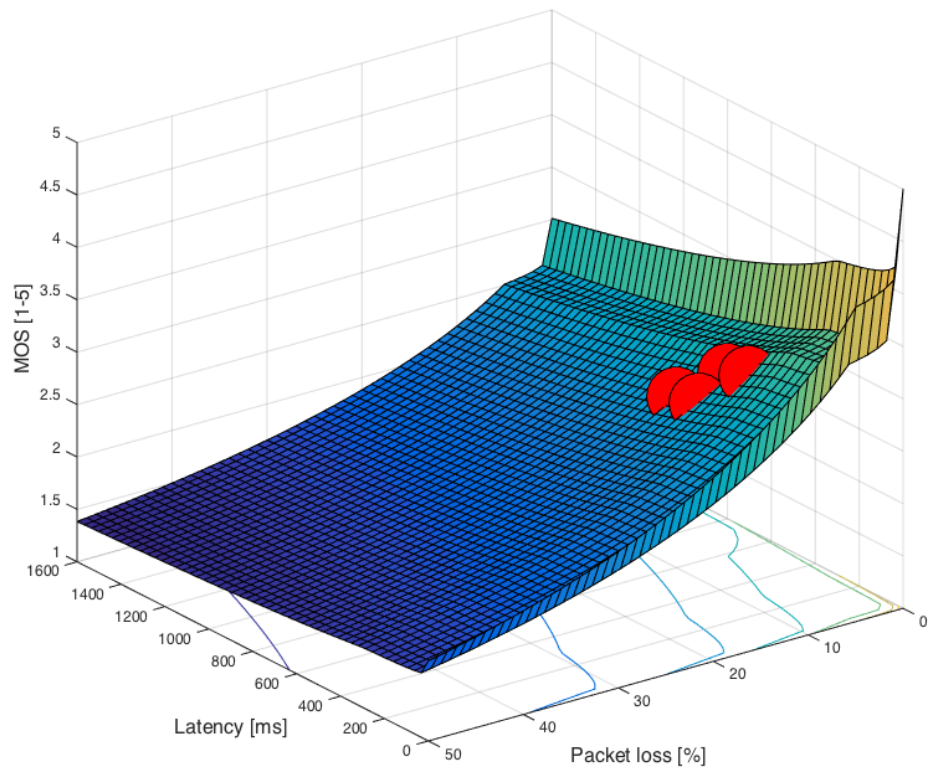

Figure 4.6: 3D-Graph of the AQX Model for Multiple Variables

sualization purposes. Those bullets should be ideally cut in half by the ${ }_{3} \mathrm{D}$ curve of the AQX model in case of a $100 \%$ accurate prediction. However, the closer a bullet is in the $3 \mathrm{D}$-curve, and the better the bullet intersects the curve, the better the prediction of the AQX model is.

\section{$4 \cdot 3$ Chapter Summary}

This chapter presented the design and execution of a QoE measurement experiment, which examined multiple different network scenarios emulations. Moreover, this setup provided the possibility to save user ratings and perform an application-specific analysis with adjustable variables being emulated and encompassing latency, packet loss, and bandwidth. The VoIP messenger - developed based on the WebRTC technology - collected a 
significant, and thus statistically solid, amount of over 500 data points in experiments with a total of 34 subjects.

The data collected was used to calculate AQX results for each scenario and respective MOS results were used to define those parameters of the AQX model for VoIP services. The evaluation performed two steps for each variable: (1) the resulting AQX model was compared to the ITU-T E-Model and the IQX hypothesis; and (2) these variables were considered in a mixed scenario with other variables.

It was shown that the AQX model in several cases shows high QoE prediction accuracy in the VoIP scenario. Moreover, an outcome of the analysis of $m$ values confirmed that they are not constant for the entire domain of a variable. Different influence factors values $m^{-}$and $m^{+}$are needed to predict accurately QoE below and above the expected value $x_{\circ}$ of a variable. Additionally, those experiments revealed that the formula for multiple variables of the AQX model produces accurate QoE prediction results, specifically for the set of measurements with mixed variables, which were performed. All these findings lead to the conclusion that AQX is a highly adaptable and precise model, which outperforms all other state-of-the-art models in the VoIP domain that was compared with. Having provided the influence factor $m$ for different services, the AQX model becomes a powerful and useful tool for VoIP SPs to predict and improve their services in terms of QoE. 


\section{5 \\ The Design to Overcome the MTR Monopoly}

$\mathrm{O}$ NCE it is possible to estimate QoE of end-users considering the service specification and end-user's preferences and priorities, it is possible to establish a QoE-based charging schema for scenarios, such as the mobile termination service. Furthermore, in markets were multiple SPs offer similar services, such as mobile calls market, and were competition is allowed or demanded, the SP that will maximize QoE of end-users may be selected to provide the given service. In mobile communication the amount that the MvNO of the callee will charge the caller's MvNO, to terminate his call into his network is termed MTR (cf. Figure 1.1). The MTR market is considered to be a monopoly (cf. Chapter 1 ). The problem of the monopolistic MTR market is also large for users that wish to receive calls in a location that is different from the home location, where the service was registered (roaming). Roaming users has to pay high prices to receive a call [143]. Thus, the ITU has put effort toward the solution of this problem [70][71][72]. Although, in June 2015 European Union (EU) agreed to scrap roaming charges from June 2017 [45], MTR in each country will continue being a monopolistic market since there will be no further MTR 
deduction in this case. Thus, it is essential to address the high MTR cost and the Mobile Termination Rates monopoly problem in general not only for roaming but also for domestic calls.

There is significant work done on MTR regulation effects that show (a) MTR are the higher the smaller the MvNO's size is (as measured through its subscriber base), and (b) asymmetric MTR regulation of only larger MvNOs in a market will induce the smaller MvNOs to increase their MTR [34]. Additionally, there is significant research on the topic of the proper selection of MTR with regulators playing a significant role defining them [25]. Furthermore, effort has been put into the analysis of business strategies, such as CPP and RPP, that MvNOs follow concerning their termination rates [62] [93]. The customer base of a single $\mathrm{MvNO}$ is significantly smaller compared to the set of total customers in every other MvNO, FNO, or VoIP provider, unless a MvNO serves more than the $50 \%$ of all end-users which is rarely the case since there are multiple MvNOs in most countries. Thereby, the majority of calls that a MvNO has to terminate in his network originate from foreign networks. Thus, for many years MvNOs revenue is coupled with high termination rates applied. This is the main motivation for MvNOs to keep regulation in this monopolistic market as low as possible.

To solve the high MTR problem dual-SIM card devices [29] [150] could be used to select on-demand the MvNO that is offering the better MTR option. However, in such case it is essential that a caller has a complete and always up-to-date knowledge of every pricing plan of each available to him MvNO. A user need to subscribe with his dual-SIM device simultaneously in two MvNOs. Usually MvNOs provide lower rates for calls between their customers (in cases those calls might also be for free), since in that case there is no termination fee involved. Furthermore, in the past, a MSISDN corresponds only to a subscriber of a specific MvNO. So, a caller identifies by the callee's MSISDN, which MvNO is serving him, and thus selects the cheaper option among his MvNOs to complete the call. However, since the Mobile Number Portability (MNP) became gradually a regulation in most countries to allow mobile subscribers to change MvNOs without changing their phone number, there is not a unique MvNO that the MSISDN 
can belong to. Nowadays, a callee can establish a new contract with any available MvNO and still can be reached by the same MSISDN. Thus, the caller needs to have prior knowledge of which MvNO is serving the callee to be able to select in the dual-SIM device the MvNO, which provides the cheaper calling rate for a specific MSISDN. Beside the callee's MvNO lack of transparency, high contract operations costs are an additional obstacle for callers wanting to use dual-SIM devices to minimize their out coming calls cost. Thus, dual-SIM devices require contracts with more than one MvNO and do not affect the Mobile Termination Rates monopoly.

Fortunately, there are two significant updates, compared to the early days of mobile communications, which enable the operation of an efficient MvNO-independent call-termination solution. This solution will have an impact toward the Mobile Termination Rates monopoly, since MvNOs will loose the control of their subscriber's call-termination procedure. Those updates are (1) the infrastructure update and (2) the mobile data introduction. In more detail, the majority of the newest mobile terminal devices, e.g., smart phones, can equally register in almost every network across the world, irrespective of the device vendor. Nowadays, smart phones are the rule and not the exception between mobile subscribers mobile terminal choice according to Nielsen [102]. Such devices have sufficient computational power, multiple network interfaces, provide positioning information, and can also support cross-platform applications, which are fully integrated within the device's UI. Thus, several procedures, like computational calculations or an exchange of data between the caller and the callee, can take place prior to a call without the calling parties experiencing any difference during the calling procedure. Furthermore, mobile data charges are nowadays decreasing [101], mobile data rates are higher and expected to be improved in the future within the next generations mobile communication networks [127].

This thesis presents here in today's mobile networks environment AbaCUS, which is (1) a MvNO-independent call-termination system in replacement of the Mobile Termination Rates monopoly, and (2) a fair charging system where the one who pays can influence the price and the QoS perceived level. Thus, this thesis overcomes the Mobile Termination Rates 
Standardization Consortiums

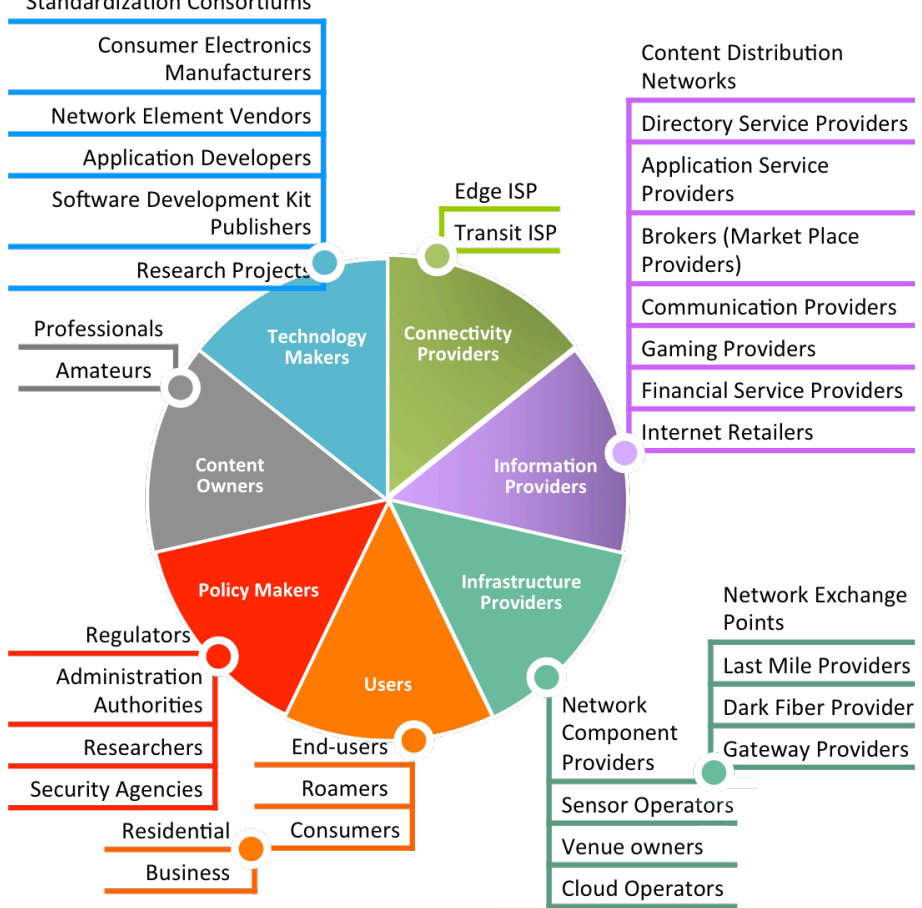

Figure 5.1: Stakeholders of the Internet Ecosystem [112]

monopoly obstacle of the MTR market, providing a fair charging solution. Furthermore, this thesis shows that the unregulated adoption by MvNOs of such a solution will have a positive impact for both end-users and MvNOs. The former is achieved by selecting the MvNO to terminate a call with a given QoS at a given price, and the latter is facilitated through the calltermination of other MvNOs subscribers.

\subsection{Stakeholders ANalysis}

The MTR ecosystem is a subset of the Internet ecosystem because only the mobile termination service is included while the Internet contains various 
services, such as VoIP, video streaming, and browsing. Thus, the Internet ecosystem stakeholders list [80][1 12] which is presented in Figure 5.1, has been used to identify relevant stakeholders of the MTR ecosystem.

A voice call is not considered as content (e.g., a movie) because each voice call between a caller and a callee is different. Thereby, there is no stakeholder in the content owner category. However, in every other category presented in Figure 5.1 there are various stakeholders for the MTR ecosystem. To compile a complete list of each stakeholder of the MTR ecosystem which is presented in Table 5.1, the seven stakeholder categories and the methodology proposed in [80][1 12] have been used. According to Project Management stakeholder characterization principles [89] and the stakeholders analysis of Table 5.1, each stakeholders' Interest, Importance, and Attitude is identified and the stakeholders' map of the MTR ecosystem is visualized in Figure 5.2. The reasoning for each stakeholder's interest, influence and attitude is presented in the respective subsection of each stakeholders' category.

\subsubsection{USERS}

During this thesis an Internet survey [134] was performed among approximately two hundred MvNO's subscribers, who belong to a diverse location, age, sex, social, and educational background to poll end-user's interest in a potential QoE-based charging for mobile voice services. As results in Table 5.2 show, it is observed that $58 \%$ of subscribers would not mind to pay more for a guarantee QoS. 74\% of those subscribers would not mind to experience a below average sound quality communication, if the total price of the call would be less than originally priced. Finally, $61 \%$ of the callers are tolerant in time delays concerning their call establishment waiting-time. Given the survey results, this thesis assumes that caller's attitude is positive and the interest in AbaCUS high because a caller will benefit either from better services, and/or lower prices (better QoE). Furthermore, callee's attitude is assumed to be neutral since there is no monetary benefit neither any loss. For the same reason, the interest of the callee is assumed to be low. 


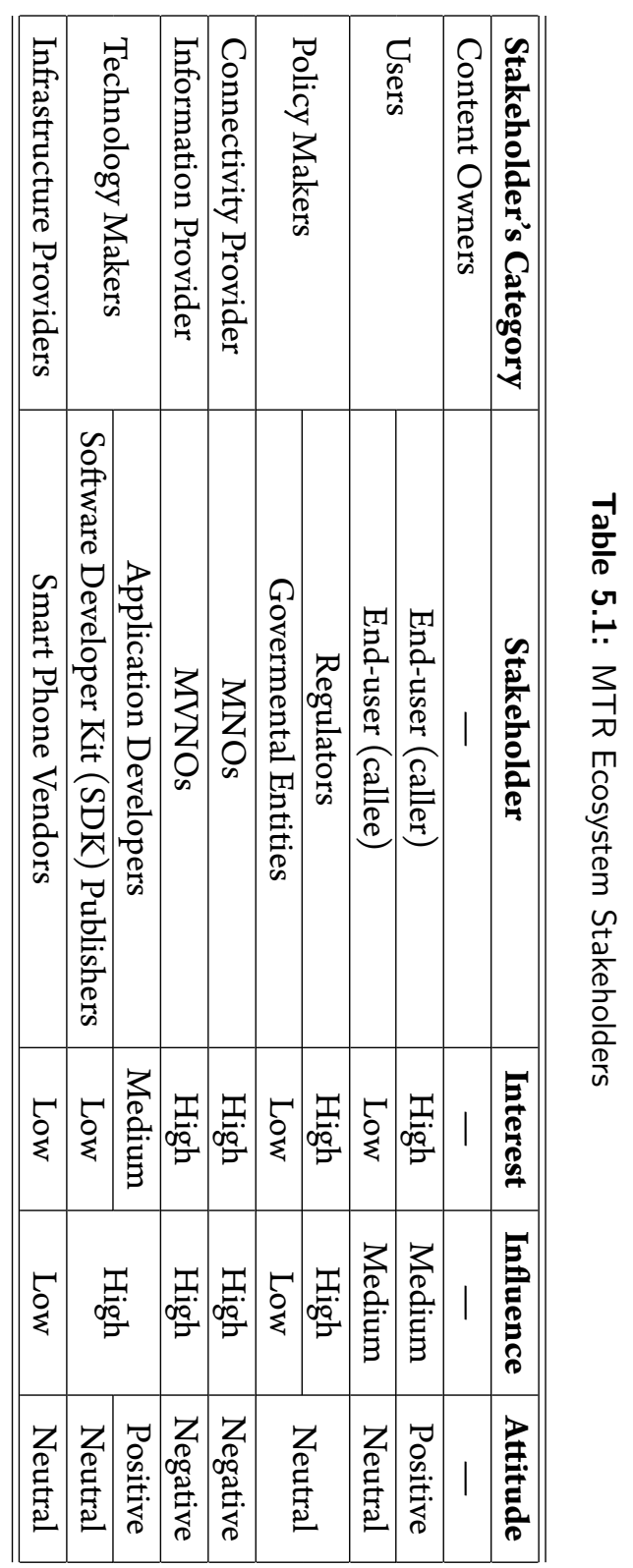


Table 5.2: Users's Behavior Survey Results

\begin{tabular}{|l|c|}
\hline \hline $\begin{array}{l}\text { Per cent of callers that willing to } \\
\text { pay extra for better QoE }\end{array}$ & $58 \%$ \\
\hline $\begin{array}{l}\text { Per cent of callers that willing to } \\
\text { accept poor QoS for a lower price }\end{array}$ & $74 \%$ \\
\hline Per cent of delay tolerant callers & $61 \%$ \\
\hline \hline
\end{tabular}

The influence of a caller and a callee in AbaCUS is assumed to be medium since high participation is essential but not mandatory for a successful termination of the MTR monopoly.

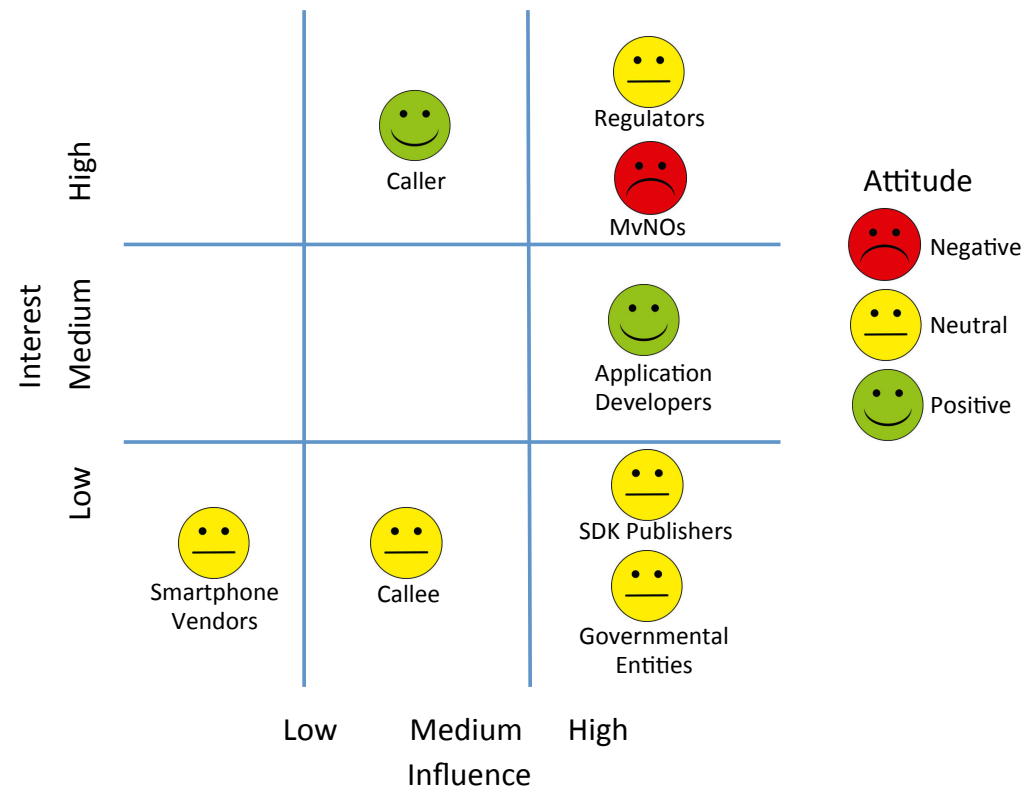

Figure 5.2: MTR Stakeholders Map 


\subsubsection{Policy Makers}

To liberate a "de-facto" market-defined monopoly, such as the Mobile Termination Rates monopoly (cf. Section 2.1), even if it is technically feasible, it would take significant time for the regulator to enforce new policies that demand from the players more investments, either in terms of infrastructure or working-time. In this thesis an interview with the Swiss Federal Office of Communications (OFCOM) [106], which is the regulation authority concerning telecommunication in Switzerland, confirmed that to liberate such a monopoly is a time costly process when regulatory actions are needed. However, OFCOM agreed that AbaCUS shows a clear impact not only on social wealth fare increment but also on MvNOs opportunity to monetize unused infrastructure.

Political action might be needed in special cases, such as the Swiss market, where governmental entities, such as the parliament, might need to take an action to enforce a new law. In such environment the regulator (e.g., OFCOM) first need to recommend the changes that are needed, and then the respective governmental entity to initiate the procedure of enforcing a new law. The latest depending on the country is an additional action needed that might complicate and slow down further a potential attempt to enforce a Mobile Termination Rates monopoly liberation. Thus, this thesis strengthens benefits for MvNOs as an incentive of a voluntary adoption of such system with a minimum regulatory and legislative demand.

Both regulators and governmental entities have high influence in AbaCUS since the system needs to be adopted to meet any local regulation/law demands. The interest of the regulator is high because AbaCUS overcomes the MTR monopoly problem and reduces the regulation demand. However, for governmental entities the interest in AbaCUS will increase only in case that the regulator recommends specific law enforcement. Finally, the position of regulators and governmental entities is neutral since there is no drawback in case the MTR monopoly cancelation is achieved. 


\subsubsection{Connectivity And Information Providers}

MNOs and MVNOs are the mobile call termination SPs and thus the key stakeholder of the AbaCUS system. MNOs are stakeholders who own mobile network infrastructure and frequency license, and MVNOs are operators who pay MNOs to use their infrastructure to provide services, such as the mobile voice service. Both, MNOs and MVNOs are providing similar services to their end-customers, but they may follow different pricing strategies although very often they operate over the same infrastructure. AbaCUS increases the utilization of MvNOs infrastructure and allows monetization of unused MvNOs resources by offering the possibility to a MvNO to provide mobile call-termination services to end-users that do not belong tot heir customer base.

There are several use-cases that show benefits for MvNOs from a liberal MTR market. (a) It is outlined at [6] that even when the network of a $\mathrm{MvNO}$ is not serving any end-users there is a high energy cost in a Base Station (BS). The BS consists of different components, such as a Power Amplifier (PA), a Radio Frequency (RF) transmitter and receiver, antennas, voltage converters, and cooling units. In macro-BSs (not femto-cells) the PA is the component with the highest energy consumption. Even when there is low load in the BS the same power supply with its fixed operation point is demanded. Thus, for a major part of the day power is wasted. In the situation of underused infrastructure a MvNO may decrease MTR to attract traffic to the network and collect MTR from end-users that wouldn't use this specific MvNO to terminate a call. This will result to the minimization of the operational cost for a MvNO. (b) Assuming that a MvNO is experiencing a technical problem or faces high load and it is not possible to serve its customers, a temporary increment of MTR will "force" end-users to use a competitive $\mathrm{MvNO}$ and they will not realize that their home MvNO would not be able to serve them and their QoE will not be decreased. Finally, (c) MvNOs can benefit from offering premium services, such as guaranteed network access in case of high network load (e.g., during a concert, a popular sports event, in crowded areas, or in New Year's Eve), to end-users that are willing to pay higher MTR. 
Despite the fact that paying for QoS can increase networks' utilization [53], the initial attitude of a MvNO is negative since the "de-facto" MTR monopoly is terminated. Furthermore, MvNOs' influence is high since a voluntary adoption of AbaCUS will eliminate any overhead for regulators and governmental entities action demand. Finally, the interest of such system is high because of the new revenue sources possibility from already existing infrastructure.

\subsubsection{Technology Makers and Infrastructure Providers}

To facilitate an automatic and on-demand MvNO selection that is needed in AbaCUS to overcome the MTR monopoly, a mechanism that can access the MvNO selection mechanism of the UE GSM modem must be accessible via the UE OS SDK. Such mechanism must be energy and time efficient as well as available for most UE OSs. As it will seen in detail in Chapter 6 such mechanism is not currently natively available by the only open source mobile UE OSs (Android). Thus, the influence of SDK publishers is high although the interest is assumed to be low and the attitude neutral since such solution is not implemented yet.

Once the automatic and on-demand MvNO selection SDK method becomes available by SDK publishers, an application developer must use the method to implement an $\mathrm{E}_{2} \mathrm{E}$ solution, such as a dialer, that uses this functionality for business purposes. E.g., the liberation of MTR market. The interest of such application developers is medium since new services will be developed, the influence is high because the implementation can affect the energy and time efficiency of the implemented MvNO selection mechanism, and finally the attitude is positive because new services mean new revenue streams.

The infrastructure providers which are the smart phone vendors have a low interest and influence in the automatic and on-demand MvNO selection mechanism because once the functionality is defined in the SDK the UE will execute the MvNO selection. There is no additional cost or revenue stream for smart phone vendors and thus their attitude is assumed to be neutral. 
Table 5.3: QoE-related Variables Identification of Mobile Calls

\begin{tabular}{|c|c|c|}
\hline Variable & Variable Type & Unit \\
\hline \hline Sound Quality & Technical & $S D, H D$ \\
\hline Guaranteed Network Access & Technical & Yes, No \\
\hline MTR & Economic & Any currency \\
\hline Call Set-up Cost & Economic & Any currency \\
\hline Charging Rate [time] & Economic & sec \\
\hline Minimum Call Duration [time] & Economic & $\mathrm{sec}$ \\
\hline
\end{tabular}

\subsection{QoE of a Mobile Call According to AQX}

To formalize QoE of mobile calls the AQX axioms and methodology is used (Section 3.1) to (1) identify QoE-related variables, (2) classify QoE variables in IV or AV, (3) identify the minimum and maximum values of each variable, $(4)$ identify the ideal/desired/expected/agreed $\left(x_{\circ}\right)$ value of each variable, $(5)$ identify influence factors $(m)$, and (6) identify importance factors $(w)$.

\subsubsection{QOE-RELATEd Variables Identification}

QoE of a mobile call can be affected by both technical and economic variables, such as the sound quality, the network access guarantees, the pricing schema of a call, the mood of the end user, and the content of the call. However, when selecting among many SPs the one that maximizes end-user's QoE only the variables that can be influenced by SPs are to look at. Thus, AbaCUS summarizes in Table 5.3 the complete set of technical and economic QoE-related variables affecting a mobile call, in respect to (1) the sound quality (SD and HD), (2) the guaranteed network access (Yes, No), the economic-related variables, such as (3) MTR (measured in any currency), (4) the call set-up cost (measured in any currency), (5) the time unit of the charging rate (sec), and (6) the minimum call duration that the $\mathrm{MvNO}$ will charge (sec). 
Table 5.4: QoE-related Variables Classification of Mobile Calls

\begin{tabular}{|c|c|}
\hline Variable & Variable Classification \\
\hline \hline Sound Quality & IV \\
\hline Guaranteed Network Access & IV \\
\hline MTR & AV \\
\hline Call Set-up Cost & AV \\
\hline Charging Rate [time] & AV \\
\hline Minimum Call Duration [time] & AV \\
\hline
\end{tabular}

\subsubsection{QoE Variables IV or AV Classification}

Following the bandwidth (IV) and price (AV) variables example concerning the classification of a variable, intuitively the more sound quality and network access guarantees an end-user has the more satisfied the end-user is and vice-versa. Additionally, the less MTR are, the call set-up cost is, the charging rate in terms of time is, and the minimum call duration is, the higher is end-user's satisfaction and vice-versa. Thus, Table 5.4 summarizes the QoE variables classification.

\subsubsection{Minimum and Maximum QoE Variable Values Identifica- TION}

The minimum and the maximum value of each QoE variable in mobile calls is well-defined for technical variables because for both variables the option in binary. E.g., the sound quality can be either $\mathrm{HD}$, or SD because those are the two available options offered by MvNOs, and the guaranteed network access can either be Yes, or No because a MvNO can either prioritize or not a call. Thus, using the unity-based normalization (cf. Equation 3.5), $\mathrm{HD}=\mathrm{Yes}=1$ and $\mathrm{SD}=\mathrm{No}=\mathrm{o}$.

For the economic-related $\mathrm{QoE}$ variables a country-specific approach is needed since in every country there is a different currency and different decision due to regulations that might need to be considered, such as the minimum call duration. Unity-based normalized values are also assumed 
Table 5.5: Min-Max QoE-related Variables Identification of Mobile Calls

\begin{tabular}{|c|c|c|c|}
\hline Variable & Min & Max & Example \\
\hline \hline Sound Quality & SD (o) & HD (1) & - \\
\hline Guaranteed Network Access & No (o) & Yes (1) & - \\
\hline MTR & 0 & 1 & $\begin{array}{c}\text { Min: } 0 € / \mathrm{s} \\
\text { Max: } 0.006 € / \mathrm{s}\end{array}$ \\
\hline Call Set-up Cost & 0 & 1 & $\begin{array}{c}\text { Min: } 0 € \\
\text { Max: } 0.20 €\end{array}$ \\
\hline Charging Rate [time] & 0 & 1 & $\begin{array}{c}\text { Min: } 1 \mathrm{~s} \\
\text { Max: } 180 \mathrm{~s}\end{array}$ \\
\hline Minimum Call Duration [time] & 0 & 1 & $\begin{array}{c}\text { Min: } 1 \mathrm{~s} \\
\text { Max: } 180 \mathrm{~s}\end{array}$ \\
\hline
\end{tabular}

for economic-related QoE variables. Since in every country the minimum and maximum economic-related variable values will be different, an example of how the minimum and maximum values might be selected is proposed in Table 5.5. The example value presented in Table 5.5 concerning the maximum MTR are selected in alignment with the highest MTR in the last decade in Europe [54]. The maximum set-up cost proposed in this thesis to be three times the minimum call set-up cost that a European MNO is charging [113], and the maximum charging rate and minimum call duration is also proposed to be three times larger than the policies that most MvNO apply [95]. The maximum values proposed to be larger than in the literature as an incentive for MvNOs and callers to receive either better sound quality and/or guaranteed network access. The maximum values in the literature assume SD sound quality and not guaranteed network access.

\subsubsection{Desired $\left(x_{\circ}\right)$ QoE Variable Values Identification}

The desired value of each variable is to be defined by end-users in their preferences when they place a call request. For technical parameters end-users must make a binary choice (either 0 , or 1 ). This is equivalent with enduser's desire to for HD sound quality or not, and guaranteed network ac- 
Table 5.6: Desired Variables Values Identification of Mobile Calls

\begin{tabular}{|c|c|}
\hline Variable & Desired Value \\
\hline \hline Sound Quality & $x_{\circ} \in\{0,1\}$ \\
\hline Guaranteed Network Access & $x_{\circ} \in\{0,1\}$ \\
\hline MTR & $x_{\circ} \in(0,1)$ \\
\hline Call Set-up Cost & $x_{\circ} \in(0,1)$ \\
\hline Charging Rate [time] & $x_{\circ} \in(0,1)$ \\
\hline Minimum Call Duration [time] & $x_{\circ} \in(0,1)$ \\
\hline
\end{tabular}

cess or not (e.g., in case of a concert, a crowded event, or New Years Eve). For economic variables the desired value must be a unity-based normalized value between the minimum and the maximum value. Thus, between zero and one. Table 5.6 summarizes the desired value options.

\subsubsection{INFLUENCE FaCtoRs $(m)$ Identification}

The influence factor $(m)$ of each variable is different for each end-user (caller) and it is calculated from Equation 3.29 and Equation 3.28 for IV variables and Equation 3.30 and Equation 3.32 for AV variables, if an ITUT MOS-compilant scale is used for QoE. Equation 3.14, Equation 3.15, Equation 3.20, and Equation 3.21 are used respectively if another scale is used. However, without loss of generality, to ease calculations the ITU-T MOS-compilant scale is assumed in this Chapter.

If there is no significant influence for a caller concerning the sound quality, the network access guarantee, or any economic variable the influence factor will be $m=0$ because this will leave QoE unaffected (MOS $=4$ $\Rightarrow$ Good) in any fluctuation of a variable (cf. Equation 3.27 and Equation 3.30. When $\left.m=0 \Rightarrow e_{i}(x)=e_{a}(x)=4 \forall x\right)$.

To demonstrate through an example how the influence factor is calculated when the fluctuation of variable affects $\mathrm{QoE}$, consider that for an $\mathrm{AV}$, such as MTR, $20 \%$ higher MTR than the desired MTR variable $x_{\circ}$ results $99 . \overline{9} \% \simeq 100 \%$ decrement of end-user's QoE (MOS:4 $\rightarrow$ MOS: 1 ). Thus, Equation 3.31 for $\mathcal{\varepsilon}=2 . \overline{99}$ and $\delta=0.2 \cdot x_{\circ}$ shows that $m^{+} \simeq$ 
$\ln (\ln 0.01 / 4 / \ln 3 / 4) / \ln 1.2=16.65$. Table 5.7 summarizes the use of each influence factor Equation for each variable type (IV, AV). Table 5.8 and Table 5.9 provide reference values for influence factors $(m)$ for different fluctuation values of IV and AV respectively and their correlation with MOS.

\subsubsection{Importance Factors $(w)$ Identification}

AQX allows to specify different importance factors $(w)$ of each variable. Those factors similar to influence factors are subject to the caller's preferences. For equally important variables their important factor must be the same. If the importance of a variable $A$ and variable $B$ is the same, then $w_{A}=w_{B}=1$. If a variable $A$ is more important than a variable $B$ the importance factor $w_{A}>w_{B}$. To illustrate the selection of importance factors for an imaginary caller assume the following importance preferences. (a) All technical variables are equally important to each other. (b) All economic variables are also equally important to each other. However, (c) economic variables are more important than technical. Table 5.10 illustrates this scenario where economic parameters influence factors are twice the technical parameters influence factors. This ratio is randomly selected since influence factors need to be calculated via a survey (end-user feedback) as it was mentioned in Section 3.4. A mathematical formula to select influence factors in the mobile calls service is not yet defined. Thus all variables assume to have the same importance $\left(w_{k}=1 \forall k\right)$.

\subsubsection{Generic QoE of a Mobile Call}

For the overall QoE of a mobile call Equation 3.33 is used to combine each variable's MOS. To illustrate the overall QoE of a mobile call a hypothetical example with specific values for (1) country-related variables, such as minimum and maximum MTR, and (2) end-user preferences, such as MTR, will be examined. 


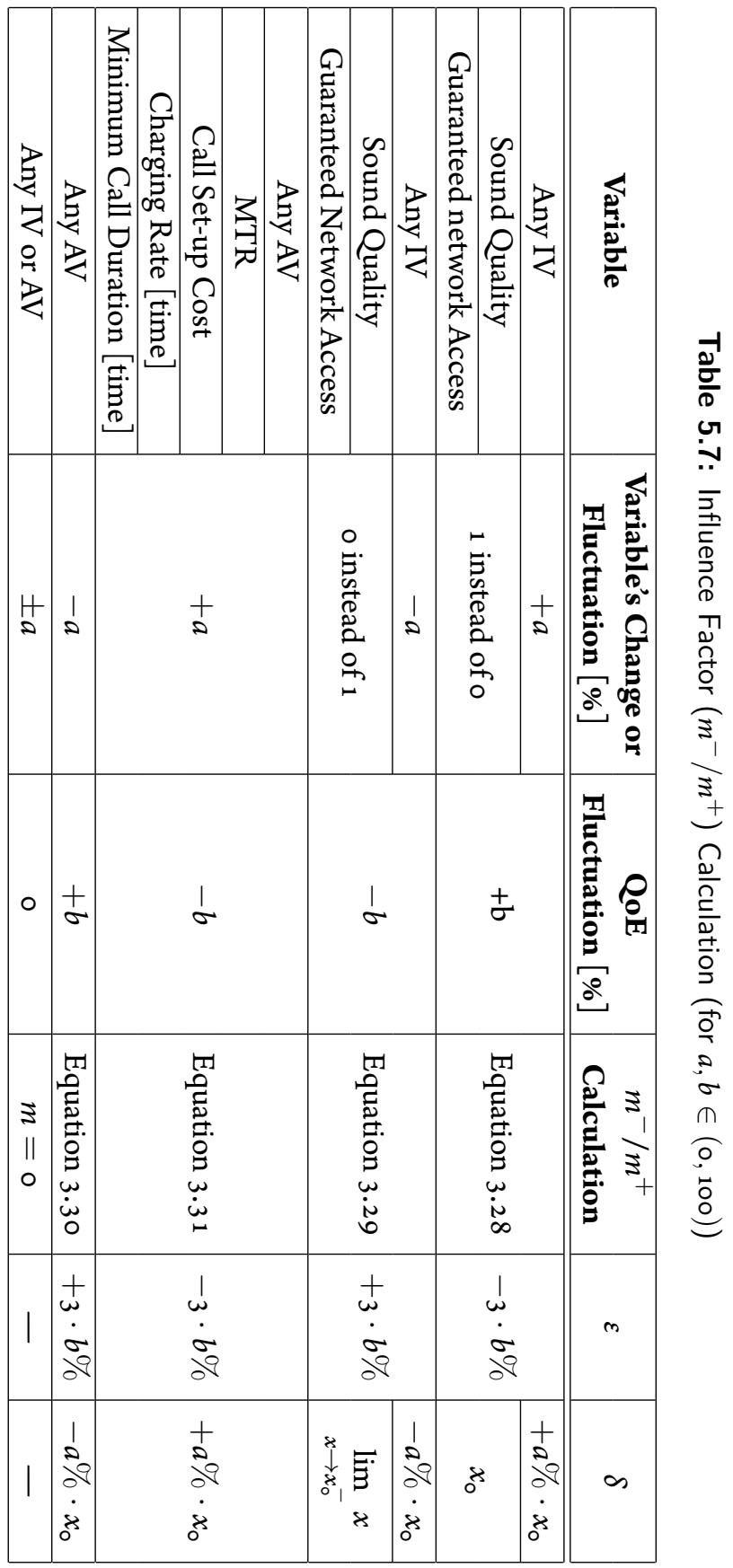




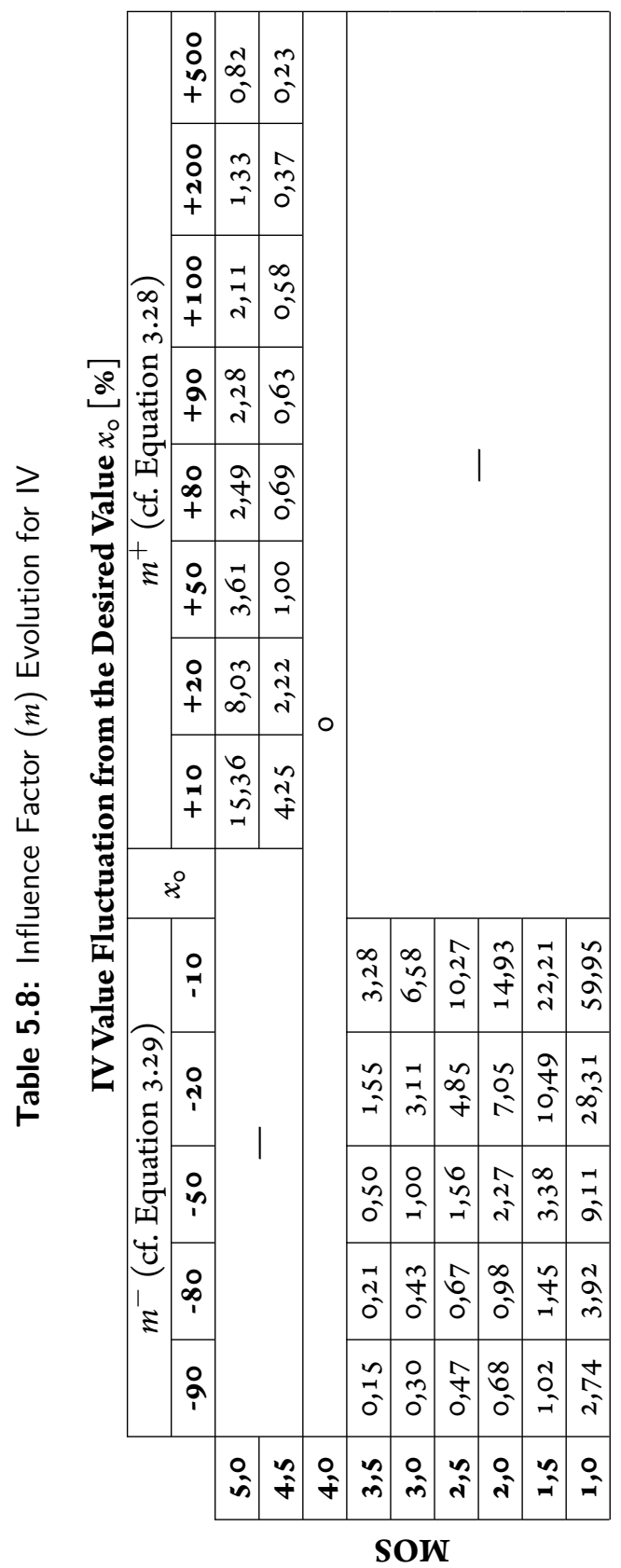


MOS

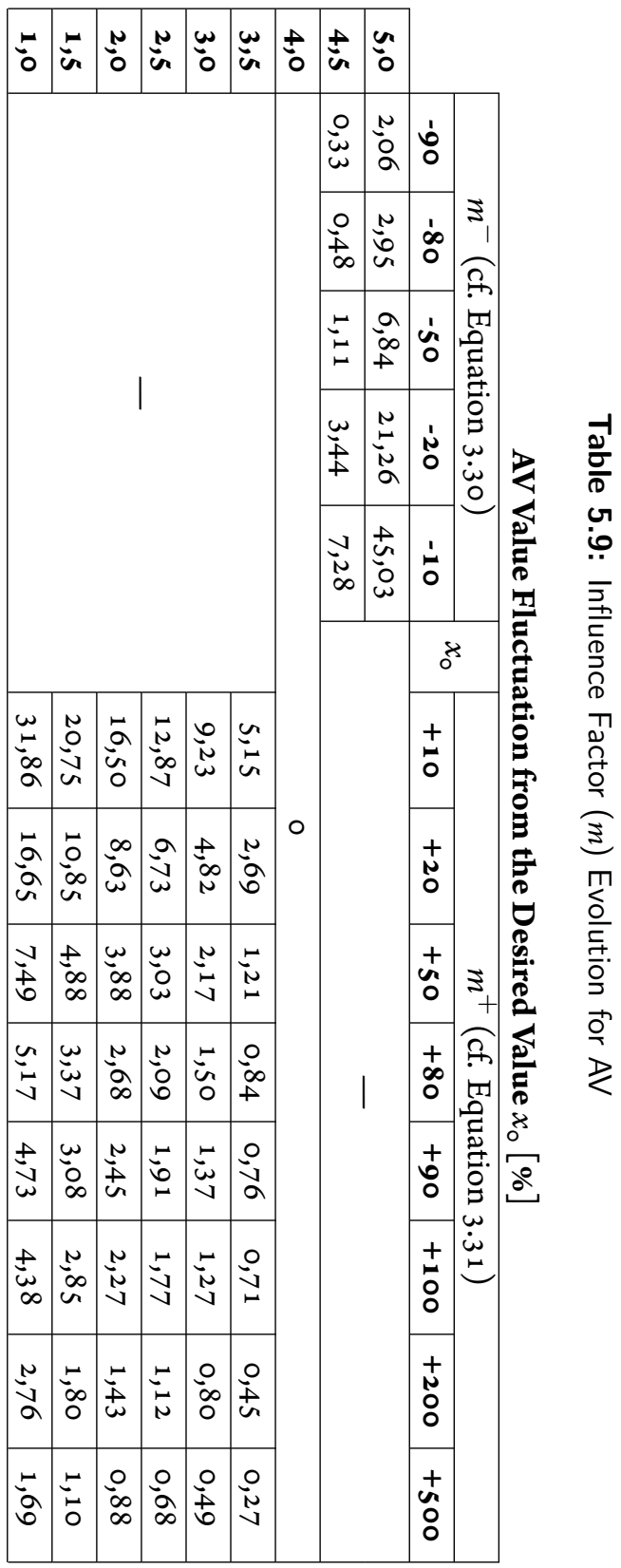


Table 5.10: Importance Factors Example for the Mobile Calls Service

\begin{tabular}{|c|c|}
\hline Variable & Importance Factor \\
\hline \hline Sound Quality & $\mathbf{1}$ \\
\hline Guaranteed Network Access & $\mathbf{1}$ \\
\hline MTR & $\mathbf{2}$ \\
\hline Call Set-up Cost & $\mathbf{2}$ \\
\hline Charging Rate [time] & $\mathbf{2}$ \\
\hline Minimum Call Duration [time] & $\mathbf{2}$ \\
\hline
\end{tabular}

Assume the minimum and maximum variable values of the example in Table 5.5. Furthermore, assume a caller that is not interested in HD voice quality and getting it would not change QoE. However, it is very important for the caller to get this call through the network. Not getting this call in case of high network load would result a low MOS. The preferred MTR is $0.003 € / \mathrm{s}$. $50 \%$ discount on MTR would satisfy the caller further (MOS: $4 \rightarrow$ MOS: 5 ), while $50 \%$ increment will decrease the satisfaction (MOS:4 $\rightarrow$ MOS: 3 ). The desired call set-up cost is $0.05 €$. $80 \%$ discount on the call set-up cost will satisfy the caller further (MOS:4 $\rightarrow$ MOS:5), while two times higher call set-up cost would dissatisfy the caller significantly (MOS:4 $\rightarrow$ MOS:2). The caller would like to get charged every two seconds for this call and even getting charged on a three seconds basis would dissatisfy the caller significantly (MOS:4 $\rightarrow$ MOS:2). The caller will accept to pay for a minimum twenty second call duration even if the call will last for less time. 90\% lower minimum call duration call will satisfy the caller further (MOS:4 $\rightarrow$ MOS:5) and 50\% larger minimum call duration is not welcome by the caller (MOS:4 $\rightarrow$ MOS:2). The desired values $\left(x_{\circ}\right)$ and influence factors $(m)$ for each QoE-related variable of the caller are presented in Table 5.11. Finally, all technical and economic variables are of the same importance for the caller.

Two MvNOs are offering two different options to the caller of the example described above. However, both MvNOs are not aware of caller's preferences. The first MvNO (A) is offering HD sound quality and guaranteed network access. The second MvNO (B) is only offering SD sound 
quality but also guarantees access to its network. Concerning (1) MTR, (2) the call set-up cost, (3) the charging rate, and (4) the minimum call duration the first MvNO offers (1) $0.004 € / s,(2) 0.01 €$ call set-up cost, (3) charging rate of $4 \mathrm{~s}$, and (4) a minimum call duration of $5 \mathrm{~s}$. The second MvNO offers respectively (1) MTR $0.005 € / s,(2)$ call set-up cost $0.03 €$, (3) charging rate $3 \mathrm{~s}$, and (4) $25 \mathrm{~s}$ minimum call duration. MvNOs offers mentioned above and results of caller's $Q \mathrm{OE}, \mathrm{MOS}$ per variable $\left(e_{i \vee a}^{1,2}\right)$ and the generic $\operatorname{MOS}\left(E_{1}, E_{2}\right)$, which encapsulates the QoE effect of all variables simultaneously, are presented in Table 5.12. Results in Table 5.12 shown that (a) none of those two MvNOs can satisfy the caller (generic MOS $\geq 3$ ) and (b) $\mathrm{MvNO}_{2}$ (B) will achieve higher QoE for the caller among those two MvNOs. Thus, MvNO 2 must provide the service to the caller if there is no other MvNO meeting better the caller's demands.

\subsection{QoS Class (QoS-C)}

The pair of the two technical variables of a mobile call in AbaCUS is called QoS-C. Since there are two available options for each technical variables, there are in total four different combinations of technical variables that a MvNO can affect. Table 5.13 summarizes the four QoS-Cs defined in AbaCUS. Each QoS class consider simultaneously two variables, (1) the sound quality (SD,HD), and (2) the guaranteed network access (Yes, No). The reason for the distinction among these classes has been taken to reach a compromise on a combination of sound quality needed and the importance of a call, while taking in account diverse QoS demands that a caller might have. To facilitate QoS-C 1 and QoS-C 2 MvNOs must have network resources reserved for callers that are willing to be charged possibly at a higher rate for such service. The proposed QoS-Cs in AbaCUS are not binding and do not need to negotiate necessarily only such binary-decision options. In the future QoS-Cs can encapsulate more, or different options, such as decision on codecs to be used in case of full VoIP over LTE services. 


\begin{tabular}{|c|c|c|c|c|c|c|}
\hline 3 & $\rightarrow$ & $\rightarrow$ & $\rightarrow$ & -1 & $\rightarrow$ & $\rightarrow$ \\
\hline${ }_{\xi}$ & 0 & 0 & $\vec{i}$ & $\begin{array}{c}\hat{i} \\
\dot{i}\end{array}$ & $\begin{array}{c}\infty \\
\infty \\
\dot{n}\end{array}$ & 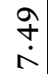 \\
\hline $\begin{array}{ll}3 & 0 \\
2 & 0 \\
z & 2\end{array}$ & + & + & $m$ & $N$ & $N$ & $N$ \\
\hline $\begin{array}{ll}\infty & 0 \\
+ & 0 \\
8 & +\end{array}$ & -1 & 1 & in & $\begin{array}{l}\circ \\
\stackrel{-}{O}\end{array}$ & 요 & ㅇ \\
\hline$\xi$ & 0 & $\begin{array}{c}\infty \\
0 \\
- \\
-1\end{array}$ & $\begin{array}{l}+ \\
\infty \\
0\end{array}$ & $\begin{array}{l}\tilde{a} \\
\dot{i}\end{array}$ & 0 & $\begin{array}{l}0 \\
0 \\
i\end{array}$ \\
\hline $\begin{array}{ll}3 & 0 \\
z & 0 \\
z\end{array}$ & + & $\rightarrow$ & $n$ & $n$ & + & $n$ \\
\hline $\begin{array}{cc}\infty & 7 \\
1 & 0 \\
0 & 1 \\
8 & 1\end{array}$ & $\mid$ & 0 & ㅇ & $\infty$ & 1 & ㅇ \\
\hline$y^{\circ}$ & 0 & $\neg$ & $\ddot{0}$ & $n$ & $\mathrm{~N}$ & 이 \\
\hline 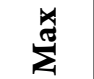 & $\neg$ & $\rightarrow$ & $\begin{array}{l}0 \\
0 \\
0\end{array}$ & ก & ○ & $\stackrel{\circ}{\infty}$ \\
\hline$\sum^{\Xi}$ & 0 & 0 & 0 & 0 & $\rightarrow$ & -1 \\
\hline$\frac{0}{\frac{0}{0}}$ & 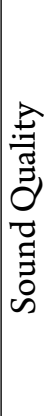 & 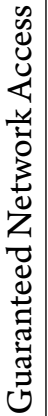 & 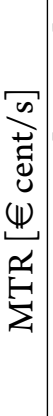 & 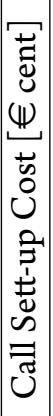 & 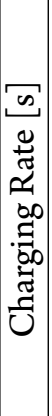 & 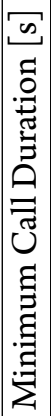 \\
\hline
\end{tabular}


Table 5.12: Two MvNOs Mobile Call Offers Example

\begin{tabular}{|c|c|c|c|c|c|c|}
\hline Variable & $\mathbf{A}$ & $e_{i \vee a}^{1}$ & $E_{1}$ & $\mathbf{B}$ & $e_{i \vee a}^{2}$ & $E_{2}$ \\
\hline Sound Quality & 1 & 4 & \multirow{6}{*}{1.02} & 0 & 4 & \multirow{6}{*}{1.05} \\
\hline Guaranteed Network Access & 1 & 4 & & 1 & 4 & \\
\hline $\operatorname{MTR}[€$ cent/s] & 0.4 & 3.33 & & 0.5 & 2.67 & \\
\hline Call Sett-up Cost [ $€$ cent] & 1 & 4.99 & & 3 & 4.75 & \\
\hline Charging Rate $[\mathrm{s}]$ & 4 & 1.06 & & 3 & 2.00 & \\
\hline Minimum Call Duration $[\mathrm{s}]$ & 5 & 4.93 & & 25 & 1.87 & \\
\hline
\end{tabular}

Table 5.13: QoS-Cs List

\begin{tabular}{|c|c|c|}
\hline QoS-C & Guaranteed Network Access & Sound Quality \\
\hline \hline QoS-C 1 & Yes & HD \\
\hline QoS-C 2 & Yes & SD \\
\hline QoS-C 3 & No & HD \\
\hline QoS-C 4 & No & SD \\
\hline
\end{tabular}

\subsection{TeR Class (TeR-C)}

The four economic variables identified affecting caller's QoE in a mobile call, (1) MTR, (2) call set-up cost, (3) charging rate, and (4) minimum call duration, are all simultaneously considered in every call. Thus, AbaCUS defines one TeR-C which contains the minimum and maximum values of all economic variables. Furthermore, the minimum and the maximum values of each variable are (a) different for every QoS-C, but (b) the same for every MvNO. The exact values of TeR-C characteristics will be selected by MvNOs representatives and approved by the regulation authority. Thus, MvNOs will compete on common TeR-C characteristics in every QoS-C.

The caller will report on-demand, (a) the desired value $x_{\circ}$ for each variable, (b) influence factors $m^{-}, m^{+}$, and (c) the importance factor $w$ for each parameter in the TeR-C, for a requested QoS-C. Thus, the overall QoE of a caller for a given offer from an $\mathrm{MvNO}$ will be estimated with $\mathrm{AQX}$ as it was shown in Section 5.2.7. Table 5.14 shows how a caller's request must look. 
Table 5.14: Caller's Preferences Request Example

\begin{tabular}{|c|c|c|c|c|}
\hline Variable & $x_{\circ}$ & $m^{-}$ & $m^{+}$ & $w$ \\
\hline \hline Sound Quality & $\circ$ & $\circ$ & $\circ$ & 1 \\
\hline Guaranteed Network Access & 1 & 1.08 & $\circ$ & 1 \\
\hline MTR [€ cent/s] & 0.3 & 6.84 & 2.17 & 1 \\
\hline Call Sett-up Cost $[€$ cent] & 5 & 2.95 & 2.27 & 1 \\
\hline Charging Rate $[\mathrm{s}]$ & 2 & $\circ$ & 3.88 & 1 \\
\hline Minimum Call Duration $[\mathrm{s}]$ & 20 & 2.06 & 7.49 & 1 \\
\hline
\end{tabular}

\subsection{AbaCUS: Auction-based Charging User-centric Sys- TEM}

Figure 5.3 illustrates the key elements of AbaCUS. A caller is flexible to use the voice-service provider of his choice, such as VoIP, MvNO, and FNO, to place a call. The caller can reach the callee by dialing directly the callee's phone number (MSISDN). In this case the host MvNO will collect MTR. In AbaCUS the MvNO that will maximize end-user's QoE will terminate the call and benefit from collecting MTR. The selection of the MvNO that will maximize QoE is taken via an auction that is hosted by the Auction Authority $\left(\mathrm{Au}^{2}\right)$, which is a neutral third party, such as a regulator or a MNP provider [132][98]. Alternative possibilities to an auction mechanism could be monitoring the available resources of MvNOs, and select the one with the most available resources to terminate a call. Is such case the load-distribution would be optimal. However, the end-user price influence would not be possible. Thus, in case of enough available resources there would be no benefit for end-users since the QoS variables would be equal on every MvNO selection, while $\mathrm{QoE}$ is influenced also by economic variables.

In AbaCUS a call can be terminated by every MvNO providing network coverage in a specific location and willing to terminate any mobile communication subscriber's call, irrespective of the provider the callee belongs to. Since the modern mobile terminal devices are multiband-compatible, there does not exist any technological boundary for this functionality any- 


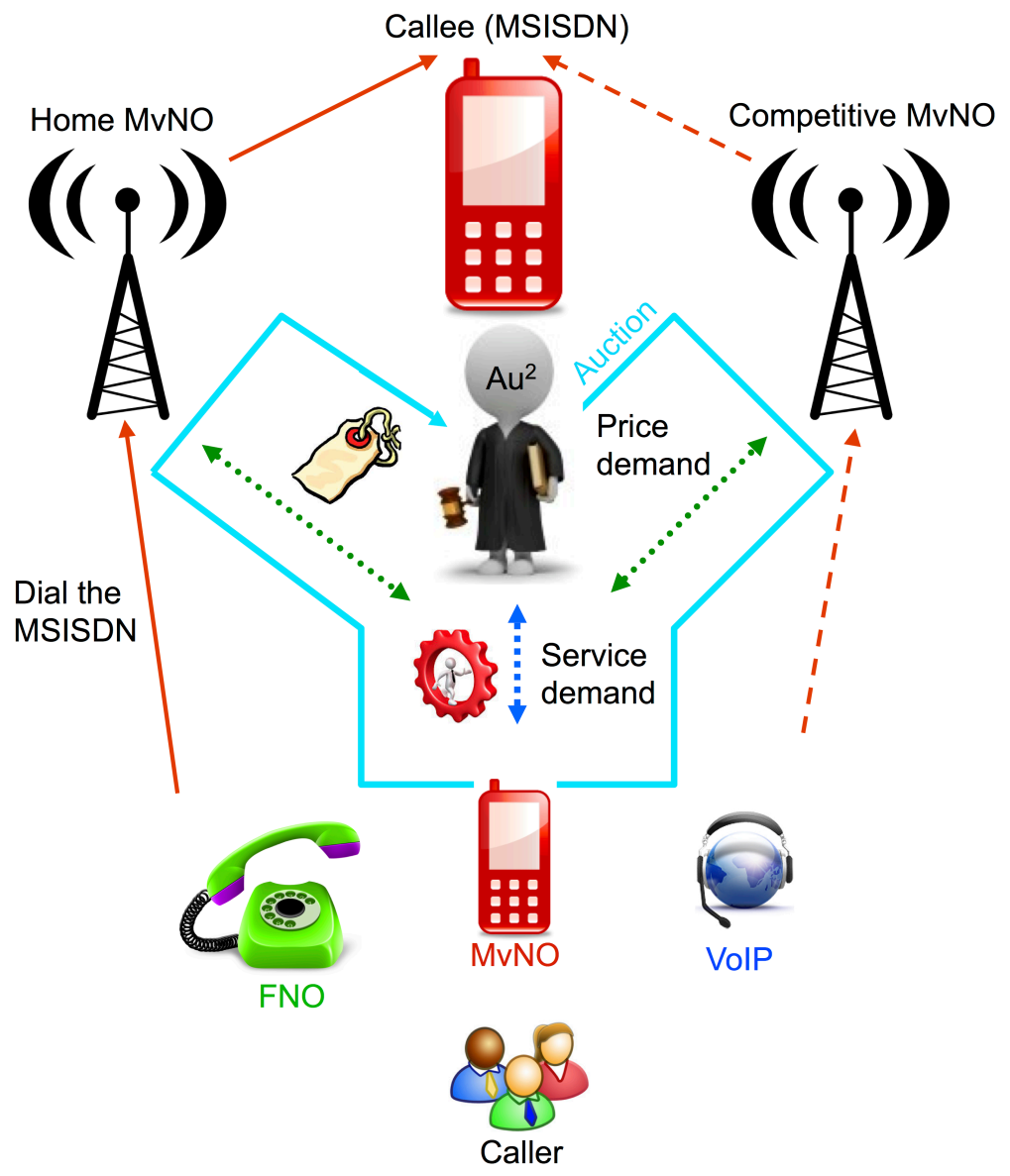

Figure 5.3: Key Elements of AbaCUS

more. Furthermore, no SIM change is required from the callee so there is no SIM-lock [ 110 ] interference with the AbaCUS call-termination MvNOindependent system. Similarly to roaming users, who can use the same device for domestic as well as abroad usage without replacing their SIM card, in AbaCUS the callee can receive a call by any MvNO that provides network coverage in his location, without the need of additional equipment.

To facilitate that a competitive $\mathrm{MvNO}$ can terminate the call of a foreign callee, a virtual MSISDN will be assigned to the callee once the callee's UE 
Table 5.15: MvNO Mobile Call Offers Example

\begin{tabular}{|c|c|}
\hline Variable & MvNO's Offer \\
\hline \hline Sound Quality & $\mathbf{1}$ \\
\hline Guaranteed Network Access & $\mathbf{1}$ \\
\hline MTR [€ cent/s] & 0.4 \\
\hline Call Sett-up Cost $[€$ cent] & $\mathbf{1}$ \\
\hline Charging Rate $[\mathrm{s}]$ & 4 \\
\hline Minimum Call Duration $[\mathrm{s}]$ & 5 \\
\hline
\end{tabular}

is parked in the new network. The caller will dial the virtual MSISDN to reach the callee. In that case the guest $\mathrm{MvNO}$ will profit from collecting MTR. Thus, in AbaCUS multiple MvNOs can participate in an auction, where a caller will request to place a call to reach a callee in a specific location while stating to the $\mathrm{Au}^{2}$ certain technical and economic preferences defined above for the specific call. Table 5.15 shows how a MvNO's call offer to the $\mathrm{Au}^{2}$ must look.

\subsubsection{AbaCUS Bidding Metric, Auction, ANd $\mathrm{Au}^{2}$}

The call termination demand is expressed by QoS-Cs and TeR-Cs, which contain values for variables related to the sound quality, network access guarantees, and economic-related variables. MvNOs announce to the $\mathrm{Au}^{2}$ the TeR-C values for each QoS-C. A call request arrive from a caller to the $\mathrm{Au}^{2}$. The call request contains the call preferences defined by the caller. Furthermore, the call request includes the respective MOSs which correspond to each QoS-C of the home MvNO of the caller given the preferences that the caller submitted. Those MOSs are calculated by the caller itself and included to the call request sent to the $\mathrm{Au}^{2}$.

On a referee role the $\mathrm{Au}^{2}$, which receives call requests from callers and from MvNOs the selected TeR-C preference per QoS-C, will indicate which MvNO between the competing ones, including the home MvNO, maximizes the caller's QoE for a given call request. The winning MvNO 


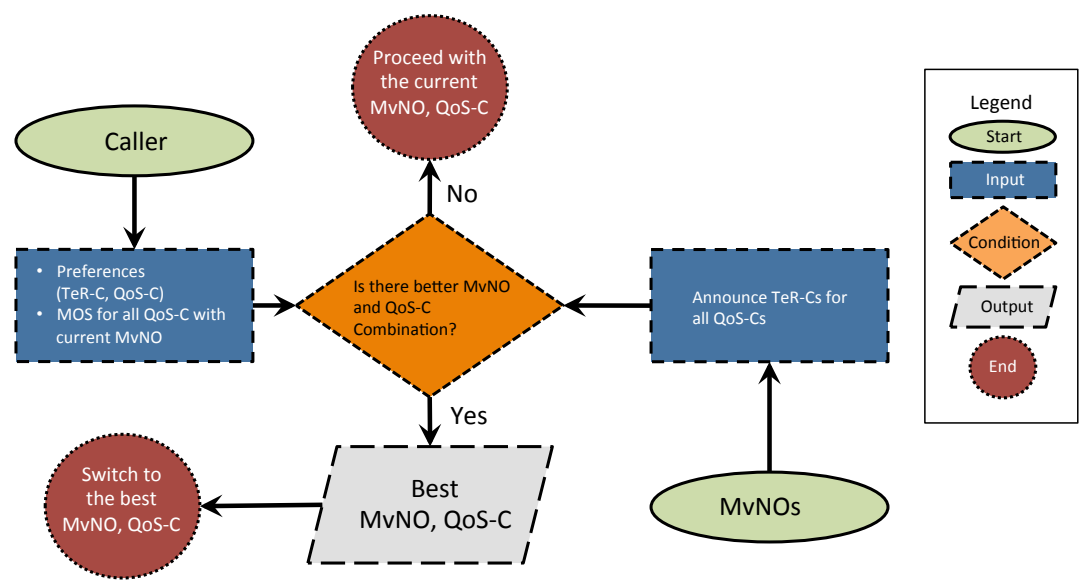

Figure 5.4: The AbaCUS MvNO Selection Algorithm Diagram

will be selected to provide the call termination service. Figure 5.4 illustrates the MvNO selection algorithm diagram.

The bidding process in AbaCUS ecosystem is described through an example. Assume that a caller request a call in the QoS-C X $(X \in\{1,2,3,4\})$. All the competing MvNOs will propose values for the TeR-C variables. Considering caller's influence factors $m$ and importance factors $w$ for all variables, a MOS will be generated for each QoS-C as it was presented in Section 5.2.7. The MvNO and QoS-C pair with the highest MOS given caller's preferences will be selected to provide the service and terminate the call. In case of a draw a MvNO among the "winning" MvNOs will be selected randomly.

In the domain of online trading and in the scope of a real-time decisionmaking feature, where the internally applied auction mechanism of AbaCUS belongs to, a single-round auction - due to urgent export result demand - is needed. Thus, based on Section 2.2 findings, the English [33] auction is proposed and the MvNO with the highest MOS of a service request is winning and provide the mobile termination service. Each MvNO has no knowledge, neither can guess, the TeR-C's variables values selection per QoS-C chosen by other MvNOs since MvNOs do not have an in- 
sight on their competitor's network status and their provisioning policies. Furthermore, MvNOs cannot predict when the next service request for a specific QoS-C will occur. Thus, it is not possible to predict a bid with a higher winning probability. To ensure that MvNOs are not interested only in specific QoS-Cs, a TeR-C proposal for every QoS-C is needed; otherwise MvNOs cannot participate in the AbaCUS auction. When a call request will be received, the $\mathrm{Au}^{2}$ will select the MvNO, which will maximize caller's QoE.

\subsection{AвaCUS Assumptions}

AbaCUS makes the following assumptions to be able to be realized in practice. (1) The regulator allows (if not allowed) the NatRoam principle so that any SIM card can camp on any MvNO. (2) The value of all economic variables must be included in the Call Data Records (CDRs) so that MvNOs can charge the caller with the respective amounts. (3) An automatic and on-demand MvNO selection mechanism is needed to facilitate callees MvNO hopping, in a timely and energy efficient manner. The first two assumptions require potential action from regulatory and standardization authorities and MvNOs. The last assumption is proven to be feasible in this thesis.

\subsection{Chapter Summary}

This chapter presented how to estimate a caller's QoE of a mobile call considering technical and economic variables. Furthermore, it presented how to overcome the MTR monopoly by selecting the MvNO to terminate a call based on caller's QoE. In the proposed MTR market, MvNOs have the opportunity to maximize the utility of their infrastructure by providing services to call receivers, who did not have access until now. Furthermore, MvNOs have the opportunity to provide premium quality services for callers, using existing infrastructure through the pre-allocation of their network resources to customers that are willing to pay more for a better and guaranteed QoS service. Finally, within AbaCUS the caller, who is the 
party paying the MTR, is also the one to chose which MvNO will terminate a call, determining a significantly fair approach, since the party paying is able to influence the total cost, the sound quality, and the network access guarantees of the call. 


\section{6}

\section{Automatic and On-demand MNO Selection Mechanism}

T 2013, 191 MNOs, which are active in 61 countries across Europe 1 [36], result in an average of three available MNOs per country. Additionally, in mobile communications there is no physical barrier (e.g., wires) that might force an end-user to stay connected with a specific MNO. Thus, MNO subscribers, due to multiple available MNOs in a location and commonly used medium in mobile communications, can hop automatically between different MNOs according to their preference. To facilitate this MNO-hopping s needed: (1) an energy and time efficient automatic and on-demand MNO selection mechanism, which is presented in this thesis for the Android platform, and (2) a large number of smart phone users that can support such this automatic and on-demand MNO selection mechanism (6.1B smart phone users by $2020[42])$.

From the MNO's perspective such a hopping attitude is driven by the fact that MNOs can benefit by offering on-demand premium services to any subscriber of any competitive MNO, e.g., high and/or guaranteed sound quality or guaranteed access to the network in case of network congestion, if the caller and/or the callee register temporarily in other network(s). In 
this case the hosting MNO can profit from collecting termination rates of the call. On one hand, MNOs can monetize some of their available network resources by attracting more users to use their services, while offering a lower than the usual price in case of low network load. According to the analysis [43], MNOs should increase their focus on new research suggestions, as the proportion of total retail telecoms revenue stemming from their current mobile services is expected to drop over the next five years. On the other hand, MNO subscribers can benefit from lower service charges and/or better QoS agreements. Despite economical benefits mentioned, an automatic and on-demand MNO selection mechanism can minimize the non-ionizing radiation of the device, especially by each time selecting the MNO with the stronger signal strength, as recently proposed by [126]. The automatic and on-demand MNO selection mechanism presented in this thesis, can be either introduced by regulating authorities and enforce the competition in the traditionally considered Mobile Termination Rates monopoly, or voluntarily adopted by MNOs that will offer ondemand premium termination services to any mobile subscriber.

A widely used automatic and on-demand MNO selection mechanism [136] must be supported by many mobile devices that can be equally used in almost every MNO across the world, to be available to many mobile subscribers. Thus, those devices must operate in multiple $3^{\text {rd }}$ Generation Partnership Project ( ${ }_{3}$ GPP) technologies such as $2 / 3 / 4 \mathrm{G}$ [19] and must have high market penetration. Smart phones fulfill those criteria; according to [103], since 2011 smart phones are the primary customer's choice. Since Android is one of the most popular platform in the smart phones market [14], the decision to implement and evaluate the automatic and on-demand MNO selection mechanism on the Android platform has been taken. Implementing such mechanism in other platforms is possible if the API is open-source such as in the Android platform. Thus, this Chapter shows that it is feasible and time and energy efficient to design and implement an automatic and on-demand MNO selection mechanism, which supports the attempt to overcome the Mobile Termination Rates monopoly obstacle. Furthermore, the path in which way to implement such a prototype in the Android platform, is also presented here. Finally, 


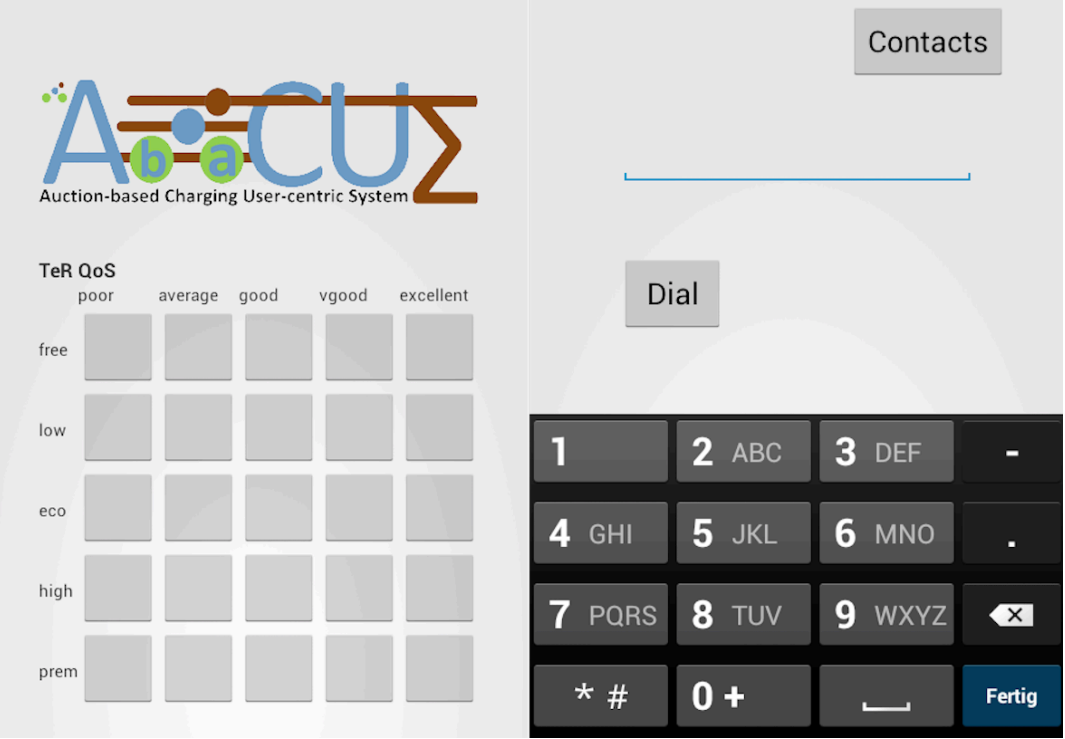

Figure 6.1: AbaCUS Dialer Application

the automatic and on-demand MNO selection mechanism has been integrated in an Android dialer application (cf. Figure 6.1) that was developed to estimate the $\mathrm{E}_{2} \mathrm{E}$ time overhead of such mechanism in AbaCUS when performing a voice call on a MNO [3].

\subsection{AT Commands Towards the Automatic and ON-DEmand MNO SELECTION}

The Attention (AT) commands interface has been a standard way to access modems as computer peripherals [129]. Generally an AT command consists of three parts. It starts with AT followed by a command and ends with the line termination character [1]. There are three different types of AT commands (Test, Read, and Set). 
Test commands test the existence of a command and check its range of parameters. The format of those commands is $\operatorname{ATxxx}=$ ?. To receive a list of available MNOs the command AT+COPS=? has to be sent to the GSM modem. The reply of the GSM modem returns a list of MNOs with the following information: (a) MNO status (o unknown, 1 available, 2 current, 3 forbidden), (b) MNO short and long alphanumeric name e.g., Orange $\mathrm{CH}$ or ORANGE, and (c) a five digit number that represents the three digits Mobile Country Code (MCC) followed by the two digits Mobile Network Code (MNC), which is the code for the network provider [125].

The read AT commands, as indicated by the name, read the current value of parameters. Set Commands are used to set new parameter values. The AT command interpreter will return $\mathrm{OK}$ in the case that the command has been successful, otherwise an error or informative result code will be returned. The MNO set AT command reads as AT+COPS=1,2,' '22801' ' '. In this command, the first integer defines the mode, with five different values (o automatic, 1 manual, 2 deregister from network, 3 set only, 4 if manual selection fails, automatic mode is entered). The second integer shows out of three possible values that format the MNO is referenced to (o long format alphanumeric, 1 short format alphanumeric, 2 numeric). Thus, if the numeric format has been chosen, the last parameter identifying the MNO is the MCC plus the MNC, e.g., 22801 for Swisscom in Switzerland.

There have been many attempts to send AT commands to Android devices, either as peripheral from a computer or directly from the device itself [51]. Within this thesis, an attempt to send AT commands and select the MNO from a computer to a Samsung Galaxy S II (SGS2) smart phone was done and it was successful. Prerequisites were that the correct GSM modem driver of $\mathrm{SGS}_{2}$ was installed on the computer and an Secure Shell (SSH) server was running in the mobile device. The device addressed over the correct port with a SSH client. However, facilitating the remote execution of AT commands in a mobile device is not secure since beside MNO selection other actions. such as dialing a number or sending an SMS can be performed. Thus, it was attempted to send a MNO change request to the device and the respective AT command to be executed by the device itself. However, the approach to send the MNO set AT command from the de- 


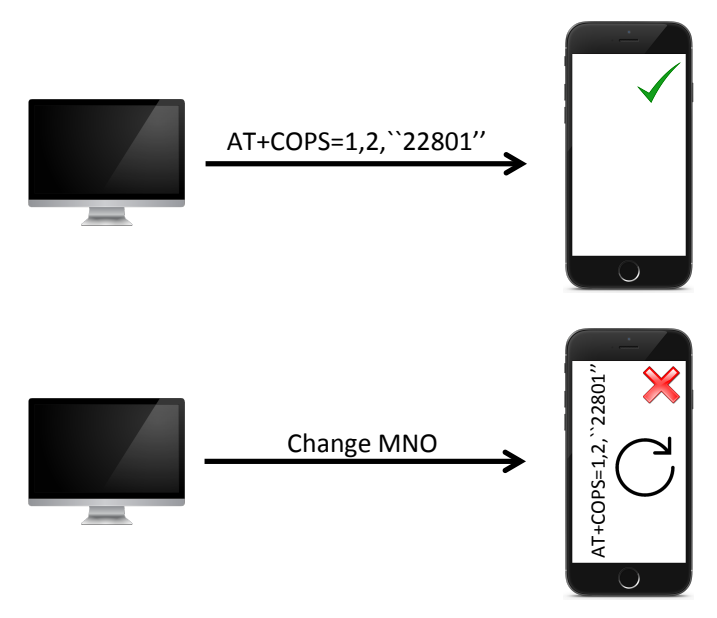

Figure 6.2: MNO Selection with AT Commands

vice itself failed (cf. Figure 6.2). Until this thesis concluded, there was no documentation of a successful MNO switching solution via AT commands directly sent from an Android device and thus an alternative method to perform automatic and on-demand MNO selection remotely is needed.

\subsection{MNO Selection Mechanism in Android}

The end user in an Android device can select the MNO in the network settings of the UI. Thus, there should be a method in the Android API performing this action once the end-user selects from the respective menu this option. However, the public Android API does not contain any methods allowing the selection of the MNO. Besides the public Android API that is accessible with the Software Development Kit (SDK), there is also an API, which is located in the package com.android.internal [52] that is not accessible via the SDK. While developing Android applications the android. jar library is referenced. In this library all classes, enumerations, fields, and methods that are marked with the annotation Qhide, from the internal package are removed. When the application is launched on a device the library framework . jar, which is equivalent 


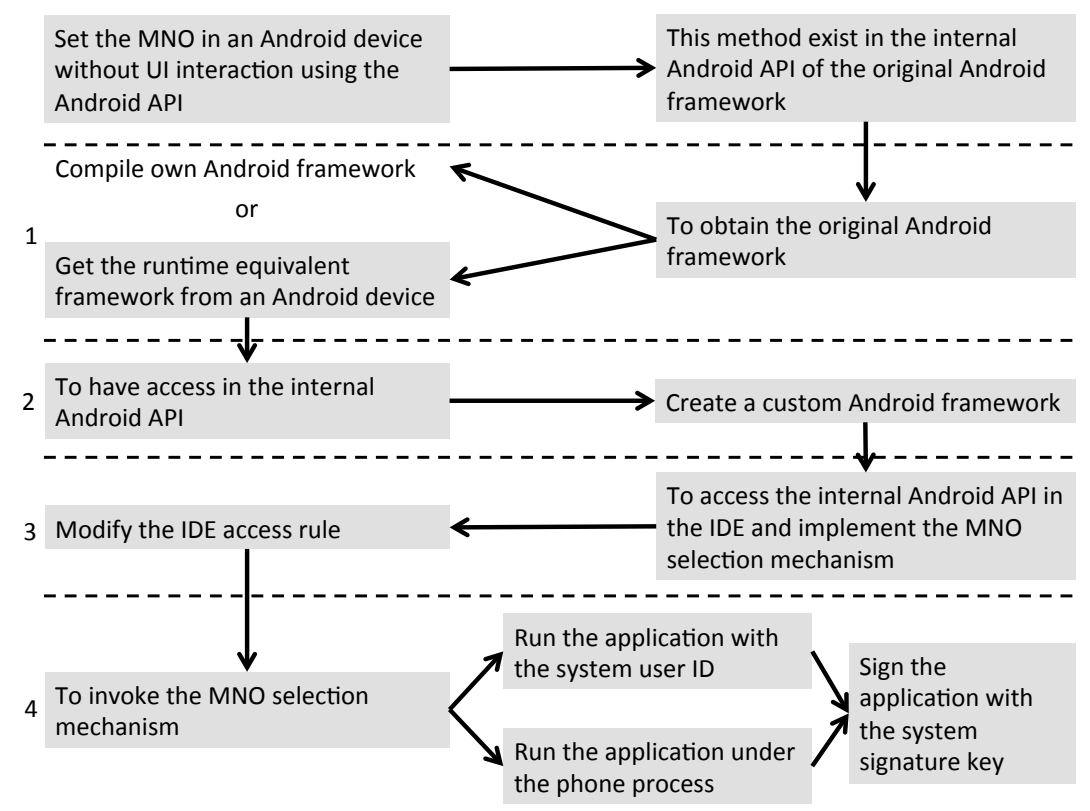

Figure 6.3: MNO Selection Mechanism Implementation Steps

to the android.jar, is loaded. However, the framework . jar library provides access with Java reflection [108] to all internal API components from the internal package.

Accessing the internal Android API requires the android. jar library to be replaced by the framework . jar. However, the Android Developer Tools (ADT) plug-in for the Integrated Development Environment (IDE) Eclipse [38] forbids the usage of any instrument in the internal package by adding an access rule to the java build path. Thus, a developer that needs to access anything from the internal API, such as the set MNO in a device method, has to do the following steps: 1) obtain the original Android framework, 2) create a custom Android framework, 3) modify the Eclipse access rule, and 4) invoke the MNO selection mechanism. Those steps are illustrated in Figure 6.3 and explained in detail below. 


\subsubsection{Obtaining the Original ANdroid Framework}

There are two different ways to obtain the original Android framework. One approach is to compile an own framework, due to the fact that Android is an open source mobile OS [11]. However, there exists another path for getting the runtime equivalent framework framework. jar which is located in the device file system at /system/framework. Within [135] the second approach has been chosen, because it is less time consuming. After the framework. jar library is downloaded it has to be extracted by the command jar xf framework.jar. If the extracted folder does not contain a file classes. dex the file framework. odex has to be downloaded from the device as well. This file has to be disassembled with baksmali.jar [6o] by the following command: java - jar baksmali framework . odex. If errors occur with the suggestion to download more odex files, missing files have to be downloaded in the same directory with the framework. odex. This will generate the Android platform related classes as smali files [22] in a folder named out. This folder has to be assembled with the command java - jar smali out. The assembled file is named out.dex and is equivalent to the file classes.dex, which has to be converted to a jar file using a tool called dex2jar [58]. The resulting jar file has to be extracted with the command jar xf framework. jar. The extracted folder contains all class files of the internal package in the folder corresponding to the package name.

\subsubsection{Creating a Customized Android Framework}

To access the internal API in an IDE, such as Eclipse, a custom framework has to be created, which contains classes and methods of the internal package. To create the custom framework the Android's SDK android.jar has to be extracted. This file is located in the Android's SDK installation folder in SDK/platforms/android-X/android.jar, where X is the API Level that is targeted at to be customized [10], e.g., level 15 for Android 4.0.4. All files being extracted from the original Android framework have to be copied into the previously extracted folder overwriting already existing files. All files in this folder have to be compressed again into android. jar 
and added to the build path. All methods of the internal package are now accessible.

The original Android framework library has to be replaced with the custom platform by replacing the original android. jar with the one created. Alternatively, the framework created can be added as new platform. To add a new platform, the entire folder of the original platform has to be copied. The original android.jar has to be replaced with the custom one. To distinguish this custom framework from the original one, a custom name and a custom API level has to be provided by adapting the file build.prop in the platform folder. The value under the entry ro.build.version.sdk has to be replaced by a desired number, which represents the API level. The ro . build. version. release value should be expanded with . extended to indicate that this is a customized platform.

\subsubsection{Modifying the Eclipse Access Rule}

There are different possible ways to modify the Eclipse access rule that prohibits the use of the internal API. The first approach is to modify the ADT source code and build it, which has not been investigated within this thesis. Another way is to modify the ADT's bytecode. Therefore, the content of the file com.android.ide.eclipse.adt_*.jar, which is located in the folder plugins of the Eclipse installation has to be extracted. The value of $*$ in the file name depends on the ADT version. In the subfolder com/android/ide/eclipse/adt/internal/project of the extracted folder the file AndroidClasspathContainerInitializer . c lass has to be opened in an editor that supports non-printable characters. The string com/android/internal/ needs to be replaced with another string such as com/android/internax/**. In turn, the folder has to be compressed with the same name as before. It has to be ensured that the internal root folder of the archive is the same as the original one, otherwise Eclipse will not recognize it. Finally, the archived folder has to be renamed to *. jar and the original ADT jar file has to be replaced with the new one. After restarting Eclipse the internal API is accessible. Another approach worked successfully only with ADT version 21 
and 22: create a new access rule that allows to use classes out of the package com/android/internal/**. Since the access rule in the subentry android. jar cannot be modified, a new access rule should be created directly below the android platform.

\subsubsection{InVoking the MNO Selection Mechanism}

Although the Android API does not provide any method to change the MNO, the class GSMPhone exists within the Android 4.0.4 source code [16]. This class contains a public method selectNetworkManually. This is part of the internal package and can be used for the purpose of the automatic and on-demand MNO selection mechanism. The class PhoneFactory provides a method to get different kinds of phone objects. To instantiate a GSMPhone object the method getGsmPhone has to be invoked [128]. Afterwards, the method selectNetworkManually can be invoked by the GSMPhone object with the required parameters OperatorInfo and a Message. OperatorInfo contains the information about the MNO to select. This includes the operator information, similar to the AT commands case, as alpha numeric long, alpha numeric short, and numeric. Here a selection could be performed, when a new OperatorInfo object with a correct MNO was created. Other values can be null or empty. Before this mechanism is usable, two further steps have to be performed: (1) run the application with a different shared user ID to prevent a SecurityException that is thrown, when protected intents [12] are sent by the methods invoked, the application has to run either with the system user ID: android:sharedUserId="android.uid.system" or with the shared user ID android:sharedUserId="android.uid.phone" [128]. This ID has to be set in the AndroidManifest.xml within the manifest-tag. (2) Run the application under the phone process. (android:process="com.android.phone") to ensure that the invocation of getGsmPhone is allowed. This attribute has to be added into the application-tag. (3) Due to the reason that the shared user ID is used by more than one applications, all applications have to be signed 
with the same certificate [13]. Thus, the application has to be signed with the system signature key. To get such a key is to run a custom Read Only Memory (ROM), which provides these certificates [55], e.g., CyanogenMod. The process of signing an application according to [17] is the following: (a) the application has to be exported as an unsigned application package. (b) the platform . x509. pem and platform.pk8 have to be downloaded, and (c) these files have to be put into the same folder as the application to be signed. Finally, before the application is sent to the device, it has to be signed with the tool jarsigner and the command: java -jar signapk.jar platform.x509.pe m platform.pk8 Application.apk signedA pp.apk, and the partition has to be remounted from a superuser with read-write privileges.

\subsection{Evaluation of the MNO Selection Mechanism}

Long delays are critical for services with a real-time network access, such as phone call establishment, and they may affect the end-user's QoE. Furthermore, energy is a critical resource in mobile communications. Thus, having an on-demand and automatic MNO selection mechanism that takes a lot of time to switch between MNOs, or consumes a lot of energy, will be practically unusable. Considering that, an evaluation in respect to the time needed between MNOs switching and energy consumption has been performed.

An evaluation of the MNO selection mechanism has to elaborate multiple successful SIM card registrations between various MNOs in the same location. However, the majority of MNOs accept in their networks only SIM cards issued by them or their roaming partners. Thus, a set-up where MNOs accept a SIM card on their network was mandatory for the MNO switching process. To facilitate this, two prepaid SIM cards issued by two different MVNOs have been used. The SIM cards selected have been chosen with the criterion that the registration is possible, while in roaming with every MNO in Switzerland (Orange, Sunrise, and Swisscom). 
One MNO hopping step includes all possible MNO switching combinations

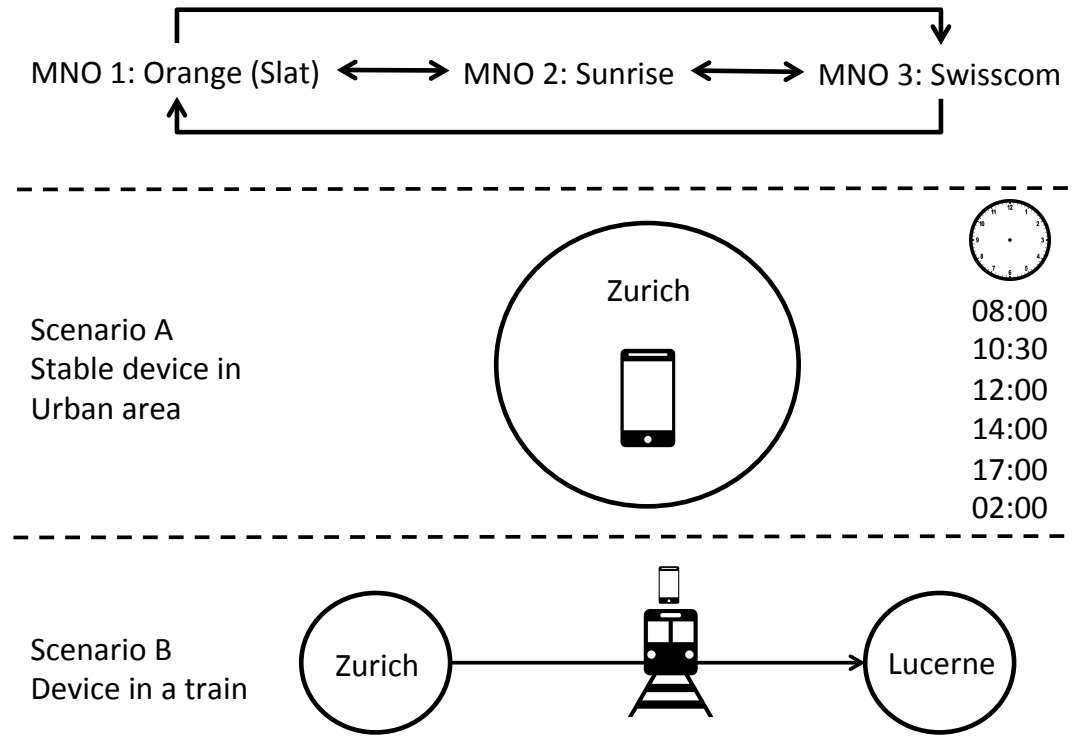

Figure 6.4: Evaluation Set-up

\subsubsection{Time Consumption between MNO Switching}

The switching time between MNOs might be affected by the signal strength, the mobility pattern of the end-user, the time of the day that the MNO switching is being performed, and the MNOs that are involved. Thus, the experiment illustrated in Figure 6.4 has been performed to evaluate the MNO switching time and find potential correlations with the parameters mentioned above. Furthermore, there exists a certain SIM card registration time overhead with a SIM card in roaming due to the fact that the local MNO needs time to authorize the foreign SIM card before accepting it within its network. To minimize the authentication overhead, the two SIM cards that have been used were registered to each available MNO prior to the measurements. A record for each SIM card would be present already during MNO switching measurements, especially in every Visitor Locator Register (VLR) of each MNO. Thus, the registration time measured had the minimum possible authentication overhead. However, since 
Table 6.1: Signal Strength Values

\begin{tabular}{|c|c|}
\hline RSSI value & Signal strength $[\mathbf{d B m}]$ \\
\hline \hline 0 & $-113 \mathrm{dBm}$ or less \\
\hline 1 & $-111 \mathrm{dBm}$ \\
\hline $2-30$ & $-109 \mathrm{dBm}$ to $-53 \mathrm{dBm}$ \\
\hline 31 & $-51 \mathrm{dBm}$ or greater \\
\hline 99 & not known or not detectable \\
\hline
\end{tabular}

these SIM cards used during the measurements were prepaid, the available balance authorization overhead could neither be avoided nor estimated. Finally, no guarantee was given that the registration process of the SIM card in roaming to a local MNO was performed with high priority.

The MNO look-up process might take more than $30 \mathrm{~s}$ [91]. Thus, the MNO list has been gathered once and their constant availability during the measurements was assumed. The MNO switching average time between the three available MNOs in Switzerland took place for the following two scenarios: (a) when the device was placed in an urban area inside a building and (b) when the device was moving on a train from Zürich to Lucerne. For a comprehensive test the switching took place between all possible MNO pairs. Thus, one test step consisted of 6 switches. This test was repeated 100 times, which led to 600 hops in total. Finally, the time needed for the entire test was measured and the average time needed per case was calculated.

To examine, if the MNO switching time is correlated to the signal strength the cell id and the corresponding signal strength have been measured in the device once, immediately after the MNO hop, and then 4 more times after every $0.5 \mathrm{~s}$. The reason why the signal strength has been captured 5 times is to confirm that its strength was stable. The corresponding cell id has been tracked to make sure that a possible signal strength oscillation is not due to a change to a different cell. According to [35] the relation between the Received Signal Strength Indication (RSSI) values and the signal strength in $\mathrm{dBm}$ is outlined in Table 6.1. 


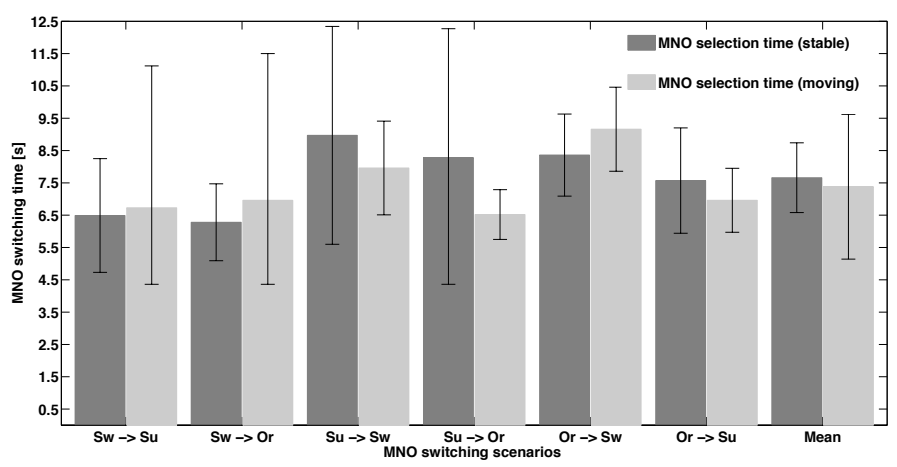

Figure 6.5: Switching Time Between MNOs

In those tests implemented the device always tries to register to an $\mathrm{MNO}$ until the attempt is successful. The measurements for case (a) was executed 6 times during a weekday in the following time frames (8:00, 10:30, 12:00, 14:00, 17:00, 2:00 hours). These time frames have been selected so that these measurements undertaken are spread during the day, when the network state (e.g., the network load) changes between rush hours. Thus, the data of the MNO selection consists of total 6 times 100 hops collected in different hours, concluding a total number of 3600 hops. The data of the MNO selection for case (b) consists of a total of 100 hops per MNO pair, reaching a total number of 600 hops, resulting in MNO switching times as shown in Figure 6.5.

The first MNO, which appears in the caption below the first set of bars, is always the MNO, where the device was registered first, and the second MNO is the one that has been switched to. Each bar corresponds to all switches performed, from the indicated MNO to another. Error bars indicate the standard deviation of all measurements. However, there is a minimum time needed to complete the 6-step SIM network registration process [109]. Thus, the assumption that the minimum MNO switching time cannot be in practice lower than the lowest value measured in this results (4.36 s) has been taken. Left bars present the average switching time between 


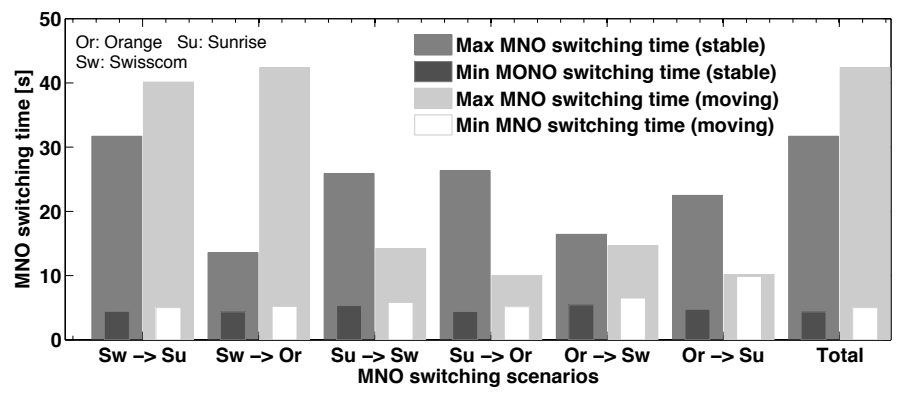

Figure 6.6: Min and Max Values for the MNO Switching Time

MNOs at the same location; right bars present the average switching time between the same MNOs while moving. The last set of bars presents the mean MNO switching time for all cases (a) and (b) in summary. The large standard deviation results from large maximum values (cf. Figure 6.6). Due to the unstable availability of MNOs while moving on a train the maximum MNO switching values appear to be much higher compared to the experiments at the same location is some cases. Furthermore, the MNO selection time shows a quite unstable behavior in some of the cases, which might be related to specific MNO's infrastructure configurations or the current capacity of the connected cell. However, the average MNO switching time is similar in both cases showing that the MNO selection mechanism performs well in every scenario.

Figure 6.7 correlates the MNO switching time with respective RSSI values $(12,8,5,3$, and 1$)$ of the new MNO. These numbers on each bar indicate the total number of times that the respective signal strength occurred out of the total test hops for scenarios (a) on the left bar and (b) on the right bar. Thus, error-bars represent the standard deviation of these measurements and they are larger in cases, where the respective signal strength has been captured only a few times. It can be seen that the signal strength is not affecting significantly the total switching time from one MNO to another. However, more measurements in a controlled environment, where 


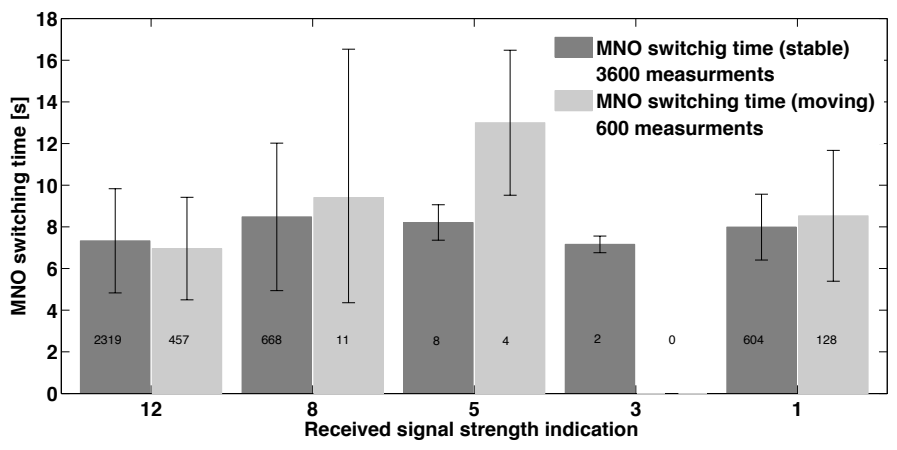

Figure 6.7: MNO Switching Time and Signal Strength Correlation

more values per signal strength are captured, can lead to more accurate conclusions.

Finally, Figure 6.8 presents the correlation of the MNO switching time for case (a) in those 6 time-slots that the experiment occurred in. It can be seen that the minimum MNO switching time is stable in every time-slot. However, the average and the maximum values are higher in the morning and early in the evening. A possible explanation of this is that the MNO's available capacity in a cell is lower when people are moving in the morning or after lunch to their offices. Furthermore, Figure 6.9 shows how many times the MNO switching time exceeds $10 \mathrm{~s}$ each time-slot. The considerably higher values around 14:00 hours are most likely due to the high network usage at that time.

\subsubsection{MNO Switching Energy Consumption}

The power consumption is critical in a mobile system. If a mechanism would absorb a large amount of available energy resources within a few network hops, the MNO switching mechanism would not be usable in practice. Hence, a detailed evaluation of the power consumption has been made. To measure the power consumption, the battery level was deter- 


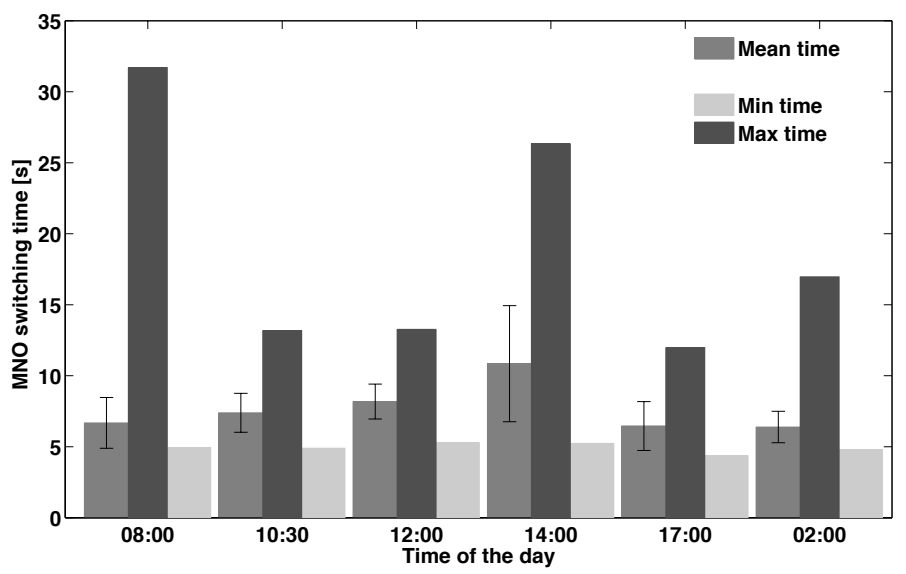

Figure 6.8: MNO Switching Time During the Day

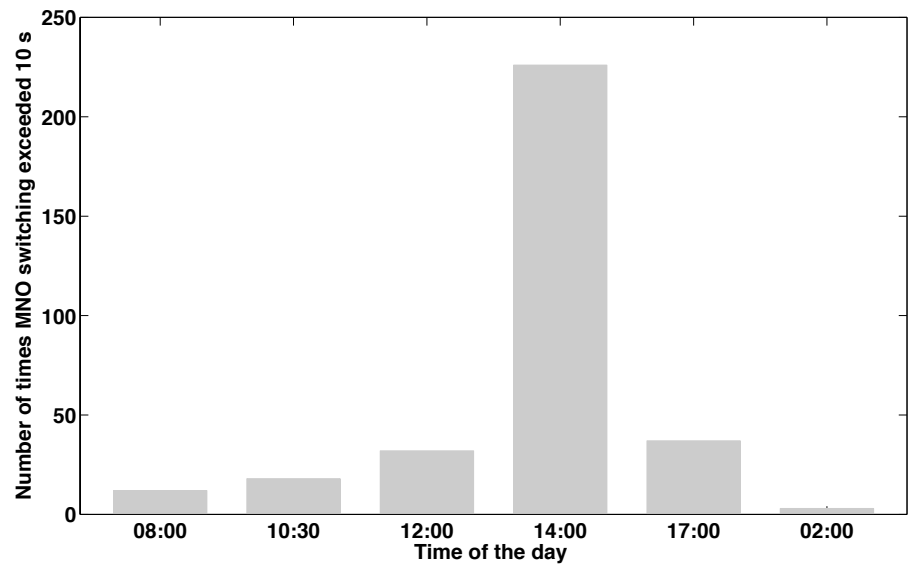

Figure 6.9: MNO Switching Time $>10 \mathrm{~s}$

mined before the test run and after the test had been performed according [15]. The difference of these levels lead to the final battery consumption in percentage of the battery energy. The assumption is that the battery health is in ideal condition. This assumption is appropriate, because the device of those measurements and its battery was new and experiments were per- 
formed in an ideal temperature for the battery [86]. This procedure was applied, since currently no Android application exists, which can measure the real battery capacity, or no application is in place, which measures the consumed energy per application accurately.

$$
\begin{gathered}
E=6.11 \mathrm{Wh} \cdot 3600 \mathrm{~s}=21996 \mathrm{~J} \\
P_{\text {tot }}=\frac{E(\mathrm{~J}) \cdot \text { Battery }_{\text {used }}}{t_{\text {experiment }}(s)}=\frac{0.14 \cdot 21996 \mathrm{~J}}{5696 \mathrm{~s}}=0.5406 \mathrm{~W}
\end{gathered}
$$

The total energy of the battery of the device used is $6.11 \mathrm{Wh}$ according to the manufacturer. Thus, the total energy that a new battery can produce is $21996 \mathrm{~J}$ (cf. Equation 6.1). During the test the display of the device remained turned off, as well as every irrelevant to the experiment process was disabled. In the test case (a), where the location was stable, the measured battery consumption was $14 \%$ and the total duration of the measurements was $5696 \mathrm{~s}$. This corresponds to the energy consumption of $3079.44 \mathrm{~J}$. To reach the total power for the MNO switching mechanism the consumed energy has to be divided by the total experiment time. However, this calculation includes also the energy needed for capturing the signal strength and the cell ID five times for each MNO switching measurement. This overhead does not affect significantly the result concerning the MNO switching mechanism, because the energy consumed on this process is small compared to the energy demanded by the MNO switching process. Thus, the results show a total $0.5406 \mathrm{~W}$ consumed power for the MNO switching mechanism (cf. Equation 6.2). The same test has been performed in test case (b), while moving from Zürich to Lucerne by train. The test lasted $7404 \mathrm{~s}$ and $22 \%$ of the battery was consumed. This corresponds to a total power consumption of $0.6536 \mathrm{~W}$. By comparing these values of both tests, it is evident that in the case the mobile device is not moving the power consumption of the MNO switching mechanism appears to be approximately $17.3 \%$ lower than the power consumption, when device is moving, most likely due to the handover energy consumption that can not be isolated. 
Table 6.2: Mobile Device Characteristics

\begin{tabular}{|c|c|c|c|c|}
\hline Consumption & $\begin{array}{c}\text { 2G max talk } \\
\text { time }[\mathbf{h}]\end{array}$ & $\begin{array}{c}\text { 2G power } \\
{[\mathbf{W}]}\end{array}$ & $\begin{array}{c}\text { 3G max talk } \\
\text { time }[\mathbf{h}]\end{array}$ & $\begin{array}{c}\text { 3G power } \\
{[\mathbf{W}]}\end{array}$ \\
\hline \hline Voice service & $\mathbf{1 8 . 3 3}$ & 0.3333 & 8.67 & 0.705 \\
\hline Stand-by & 710.00 & 0.0086 & 610.00 & 0.010 \\
\hline \hline
\end{tabular}

Table 6.3: Power Consumption Evaluation

\begin{tabular}{|c|c|}
\hline Process & Power $[\mathbf{W}]$ \\
\hline \hline Talk 3G & 0.7050 \\
\hline MNO selection moving & 0.6536 \\
\hline MNO selection stable & 0.5406 \\
\hline Talk 2G & 0.3333 \\
\hline \hline
\end{tabular}

The MNO selection mechanism power value is comparable to the power consumption of the talk mode in $3 \mathrm{G}$ networks, which is calculated considering manufacturer's maximum stand-by and talk-time in $2 \mathrm{G}$ and ${ }_{3} \mathrm{G}$ networks, as shown within Table 6.2. Thus, the power consumption of an MNO switching compared to other services, such as a hone call is shown in Table 6.3.

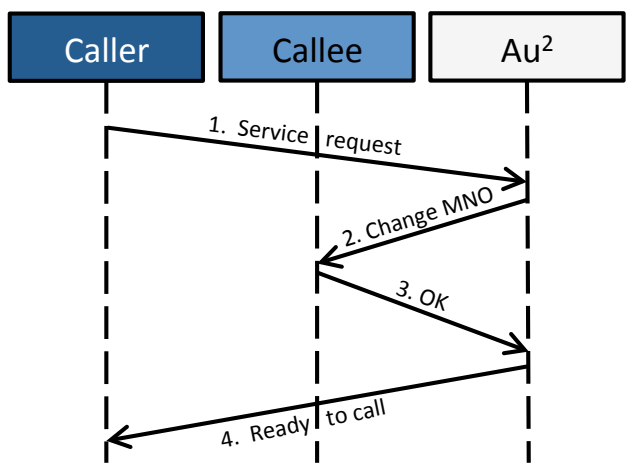

Figure 6.10: AbaCUS Service Request 


\subsection{AвaCUS E2E Calling-time}

To estimate how long it takes to be established a phone call in AbaCUS, the $\mathrm{Au}^{2}$ server, which return the MNO that will terminate a call, has been mocked in a local network. A phone call according to the AbaCUS protocol (cf. Figure 6.10) took place. The total time from the initiation of the call until the callee's phone was ringing was measured. The $\mathrm{Au}^{2}$ mock server returned the winning MNO that was selected for simplicity purposes to be always the same since it was already shown that the average MNO switching time is not correlated to the participating MNOs. Since the average MNO switching overhead has been calculated during the two scenarios in an urban area and in a train, and the $\mathrm{E}_{2} \mathrm{E}$ mobile-to-mobile $\left(\mathrm{M}_{2} \mathrm{M}\right)$ call duration has been measured by the the regulatory authority for electronic communications and postal services in Portugal ANACOM [8] and expected to be up to $17 \mathrm{~s}$ in [7], the $\mathrm{E}_{2} \mathrm{E}$ AbaCUS test call has been done 30 times. Ten times the callee's device had to switch from Orange to Swisscom and ten times it had to switch from Sunrise to Swisscom. Additionally, in ten more cases the time was measured when the callee's MNO did not have to change, because it already was Swisscom. These results are summarized in Figure 6.11. The different bars indicate the average time consumed for a call termination, where error bars are representing the standard deviation of all measurements. The average time in the case where no MNO change happened is still comparable with the normal dialing case where AbaCUS is not involved. Thus, is shown that for the AbaCUS protocol the average time of a call establishment process mainly depends on the dialing time. The difference of the calling time that a MNO switching is involved, to cases where the MNO has not been switched, corresponds to the average MNO selection time that has been evaluated in Subsection 6.3.1 A above. The first MNO in the caption below the bars indicates to which MNO the callee's device was registered before the call. The second MNO is the winning one, which the callee's device had to switch to. The third bar means that the callee's device was already registered to the winning MNO, and the last bar shows the average time when dialing with the traditional dialer without AbaCUS. Those results are aligned with the $\mathrm{E}_{2} \mathrm{E}_{2} \mathrm{M}$ call estab- 


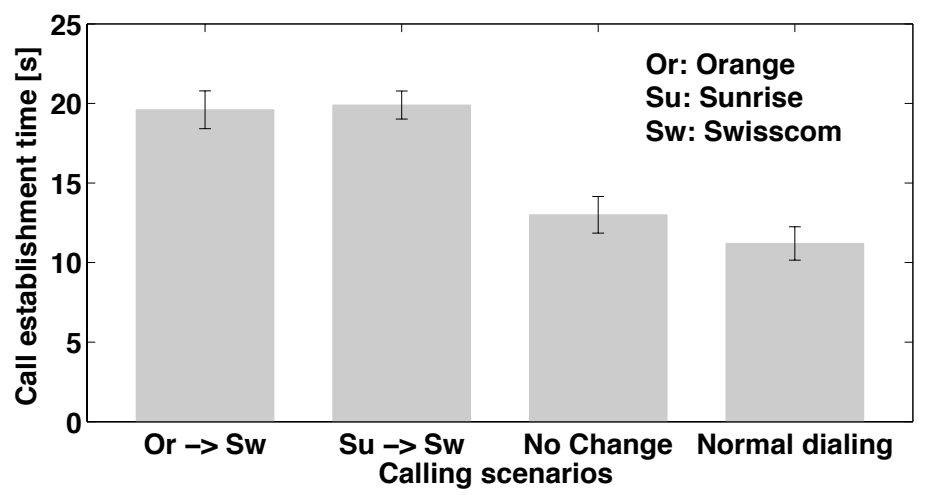

Figure 6.11: AbaCUS E2E MNO Calling-time

lishing times presented at [7], when adding the MNO switching overhead of the mechanism that was implemented and evaluated in this thesis.

\subsection{Chapter Summary}

Summarizing, an automatic and on-demand MNO selection mechanism for the Android platform had been designed and implemented. The evaluation of the MNO switching mechanism and the AbaCUS E2 $\mathrm{E}$ calling process showed that the time consumption as well as the communication to the server was negligibly low to allow an on-demand-based MNO selection for call termination purposes. For both end-user-movement scenarios (the stable mobile user in an urban location or moving in a train) it was shown that the MNO switching time is independent of those MNOs involved and on average it is expected to be below $10 \mathrm{~s}$. Secondly, the power consumption of an MNO switching is in the same dimension of the power needed, when a phone call is performed. To demonstrate key requirements, an Android application has been implemented, which makes use of the MNO selection mechanism. An automatic and on-demand MNO selection mechanism is proven to be a realistic and feasible requirement assumption for AbaCUS even being implemented without having access to the Android API. 
The existing knowledge on how to use the internal Android API has been combined to gain access to methods in connection with the GSM modem, which the open Android API does not provide. Thus, an automatic and on-demand MNO selection mechanism has been designed, implemented, and tested successfully. However, this workaround is not the best method, since the internal API is not listed and also may change in the future. Nevertheless, this thesis showed that such a mechanism is doable and realistic from an energy and time perspective, especially when it is compared to other type of services, such as the traditional phone calls. Thus, the source code of the developed mechanism in [135] can be found at [2]. However, since such a mechanism could be used in the Mobile Termination Rates monopoly liberation, or it could open the window for additional services, such as on-demand QoS-guaranteed services, an automatic and on-demand MNO selection mechanism should be published in the open API by all smart phone vendors. 


\section{Summary, Conclusions, and Future Work}

UMMARIzING, this thesis has proposed and investigated several key as-
pects involved in allowing a fully-competitive MTR market in countries where the CPP principle is applied. The path to select a MNO to provide the mobile termination service in a liberal MTR market that is proposed in this thesis, is to select the MNO which maximizes caller's QoE. Thus, it is essential to determine QoE in a way that can be estimated/predicted before a service is provided. On this path the generic Axiomatic QoE model AQX is developed here. AQX novelty is (1) that it can consider multiple and diverse economic and technical parameters, and (2) that estimates a per user personalized $\mathrm{QoE}$ of a service, considering influence and importance factors of each variable affecting $\mathrm{Q} o \mathrm{E}$. The validity of this generic QoE model has been challenged in the VoIP scenario where the comparison with state of the art QoE models showed that AQX outperforms.

QoE estimation of the mobile termination service considers the QoS-C which is one set of technical variables, and TeR-C which is a set of economic variables. On the technical side (a) the preferred sound quality and (b) the network access guarantees. On the economic side the influence and importance factors of (1) MTR, (2) set-up cost, (3) charging rate of a call, 
and (4) minimum call duration are considered. MvNOs that are competing to terminate a call propose a set of values for the economic variables for all QoS-Cs and the one that maximized caller's QoE is selected to provide the service and collect the MTR.

The stakeholders involved in the mobile call process are identified and for each stakeholder its incentives and actions needed have been identified. It was shown that liberating the MTR market is essential since (1) the caller who is the paying party will be able to define and influence the overall cost and quality of a call, (2) MvNOs are able to terminate calls for callees that do not belong to their networks and possibly get access to new revenue streams, and ( 3 ) the regulation and governmental authorities will be able to liberate a traditionally consider monopolistic market. Finally, a prototype automatic and on-demand MNO selection mechanism assumed in AbaCUS, has been implemented and proved to be usable in real life scenarios considering ( 1 ) energy consumption, and (2) time consumption per MNO hop. Thus, a liberated MTR environment is illustrated in this thesis, proving the hypothesis of this thesis for a negative answer to the primary research question "is the MTR a de-facto monopoly?". However, answering the first research question raised further research questions which have been tackled accordingly.

\subsection{OvERCOMING THE MTR MONOPOLY}

Once the MTR monopoly is not a "de-facto" monopoly the research question of how to overcome the current monopoly is seeking for an answer. AbaCUS is the answer to this question since it is the design to overcome the MTR monopoly. AbaCUS supports an auction procedure for every call separately, establishing like that a dynamic, live, and on-demand competition in the MTR market. QoS-guaranteed services and influence of the overall caller's cost, by the caller itself, are also supported in AbaCUS. Furthermore, the caller has to set only preference without any knowledge on MNOs MTR charging policies. Additionally, MvNOs can act independently, since it is optional to participate in AbaCUS auctions, or adopt other approaches. The AbaCUS MvNO selection process which is an En- 
glish auction mechanism defines the MvNO that will maximize caller's $\mathrm{QoE}$ to terminate a call and benefit from collecting MTR. Thus, two more research questions have been answered in this thesis (a) concerning the technical feasibility of AbaCUS, and (b) concerning the selection criterion of MvNOs in the liberal MTR environment.

\subsubsection{Automatic and On-Demand MNO Selection}

The research question "is it technically feasible from an energy and time efficiency point of view to implement an automatic and on-demand MNO selection mechanism?" assumed to be positively answered. The hypothesis was proved valid since (a) an automatic and on-demand MNO selection mechanism has been implemented in this thesis for the Android platform by accessing the internal Android API, (b) the MNO switching time could be completed in a timely manner while the mobile device was still in urban areas or traveling in a train irrespective of the participating MvNOs and their signal strength, and (c) the MNO switching mechanism energy demands was proven to be in the same order of magnitude with a voice call (between a $2 \mathrm{G}$ and a $3 \mathrm{G}$ call).

\subsubsection{QOE FORMALIZATION}

AbaCUS answers the research question "how to select which MNO will provide the service?" by selecting the MvNO that will maximize the caller's QoE, considering both technical and economic variables. The next research question concerning the QoE calculation was the Axiomatic QoE model AQX. The QoE model proposed in this thesis formulates QoE in six steps: (1) identify all variables tha affect QoE and can be affected by the SP, (2) characterize those variables (isotonic/antitonic), (3) select the ideal, desired, expected, or agreed value of each variable, (4) considering the service specification identify the best and the worst values of each variable, (5) identify the influence factor of each variable, and (6) identify the importance factor of each variable.

To answer the research question "why is AQX better than other QoE models?", AQX has been compared to other state of the art QoE models in 
the VoIP scenario and proved to capture end-users QoE more accurately. Also, the differentiation between IV and AV enables to quantify which/if some correcting actions could possibly revert the negative effect of a $\mathrm{QoE}$ variable fluctuation. However, AQX is not service-specific model and thus it could be applied in the mobile call scenario as well. The MOS that is generated by AQX is used in AbaCUS as a bidding metric in an English auction that defines which MNO will maximize a caller's QoE, considering the service characteristics, sound quality and network access guarantees, and several economic variables.

\subsection{CONCLUSIONS}

The last research question "what are the MTR stakeholders incentives to adopt AbaCUS?" is answered in the conclusions of this thesis. Callers are able to maximize their QoE by influencing the $\mathrm{E}_{2} \mathrm{E}$ cost of a call and QoSrelated parameters, such as the sound quality and guaranteed network access. Thus, callers can benefit from lower prices and/or better services. MNOs can offload traffic in congested network scenarios by increasing the requested values of TeR-C's variables, and when there are enough unused resources and the TeR-C variables can be offered in lower values to "attract" callers in the network and monetize idle and costly existing infrastructure.

A demand from technology makers, such as mobile OSs SDK providers, is to provide public methods that allow to select the MNO without any interaction with the UI. The implemented automatic and on-demand MNO selection mechanism could and must be multi-platform available to facilitate AbaCUS. A properly implemented automatic and on-demand MNO selection mechanism will also be possibly more energy and time efficient because the internal GSM modem methods that perform MNO's selection is possible to be available to software developers and optimized for every platform.

Policy makes, such as regulation authorities in an AbaCUS moderated market will have an observer role instead of their juristic role today. Less regulation demands will result in a competitive market with all those benefits that the AbaCUS approach implies. Given the survey results achieved, 
it is shown that AbaCUS is an approach with a strong potential to be accepted by MvNOs subscribers, since the majority of subscribers are positive toward a potential price and QoS correlation. Finally, the automatic and on-demand MNO selection mechanism is powerless unless a SIM card can be registered in any MNO. Thus, either the MvNOs must voluntarily allow such action, ot policy makers must enforce it.

This thesis provides (1) an operational prototype, (2) a suitable and well-detailed QoE modeling, (3) evaluation of its applicability in various scenarios such as VoIP, and (4) detailed selection of all the necessary QoE parameters of the introduced QoE model in the MTR market. Thus, the $\mathrm{E}_{2} \mathrm{E}$ path, including technical requirements, towards the MTR monopoly termination is presented in detail.

\subsection{Future WORK}

It is essential to examine more variables that affect callers $\mathrm{QoE}$ in the mobile termination service market. Also, define via a more extensive market analysis new potential QoS-Cs, might reveal QoE-related variables that increase the social welfare and MNOs infrastructure utilization. Furthermore, a mechanism to protect dissatisfied users that paid, but never perceived the expected QoS, is needed. Additionally, the accounting system that will handle the updated CDR for charging and billing purposes is a major engineering challenge to tackle. A potential direction would be to use soft-SIM cards instead of dual SIM devices which consume twice the energy needed, due to the two active in parallel mobile interfaces.

The exploration of further auction types to be used by $\mathrm{Au}^{2}$ could follow to quantify potential better improvement of infrastructure utilization and social wealth-fare increment. However, the chance of such monopolistic environment to change is relatively low because this thesis proposed a solution that liberates the Mobile Termination Rates monopoly in the CPP environment which is a market-established monopoly since the beginning of mobile telecommunication. Nevertheless, this thesis considers new networks and technologies to show that when the parameters of a problem change, past solutions should be reevaluated. Thus, MTR must not be con- 
sidered a de-facto monopoly anymore, and future services, such as VoIP services over mobile networks must consider a more competitive environment in the principles that AbaCUS proposes.

The efficiency of AbaCUS in other markets, like the VoIP and the fixed telephony market, might also be examined. Thus, policy makers in future mobile networks should put the effort towards liberalization of marketdefined monopolies, such as spectrum access, and maybe introduce AbaCUS principles at a lower layer. E.g., on the physical layer and Media Access Control (MAC) layer by introducing more flexible spectrum access, such as cognitive radio approaches. 


\section{References}

[1] 3rd Generation Partnership Project (3GPP), "Technical Specification Group Terminals, AT command set for User Equipment (UE) (Release 6)," June 2003.

[2] AbaCUS, "Auction-based Charging and User-centric System," URL: http: //www.abacusproject.eu/, March 2015.

[3] ——, “Callee's Mobile Network Operator (MNO) Change," URL: https: //youtu.be/19Wq8SEWoXs, November 2015.

[4] M. Alreshoodi, J. Woods, "Survey on QoE $\backslash$ QoS Correlation Models For Multimedia Services," International Journal of Distributed \& Parallel Systems, Vol. 4, No. 3, pp. 53-72, May 2013.

[5] Amazon, “Amazon Elastic Compute Cloud (Amazon EC2)," URL: http: //aws.amazon.com/ec2/, March 2015.

[6] A. Ambrosy, O. Blume, H. Klessig, W. Wajda, "Energy Saving Potential of Integrated Hardware and Resource Management Solutions for Wireless Base Stations," 22nd IEEE International Symposium on Personal Indoor and Mobile Radio Communications (PIMRC 2011), Toronto, Canada, September 2011, pp. 2418-2423.

[7] ANACOM, "Mobile Communications Systems GSM/UMTS Quality of Service Assessment," URL: http://tiny.uzh.ch/uK, November 2011.

[8] ——, "The Regulatory Authority for Electronic Communications and Postal Services in Portugal," URL: http://www.anacom.pt/, November 2015 .

[9] ANATEL, "Agencia Nacional de Telecomunicacoes," URL: http://www. anatel.gov.br/hotsites/anatelgrandeseventos/en/home/index.html, March 2015.

[10] Android, “API Level," URL: http://developer.android.com/guide/ topics/manifest/uses-sdk-element.html\#ApiLevels, March 2015. 
[11] ——, "Downloading and Building Android," URL: http://source. android.com/source/building.html, March 2015.

[12] ——, “Intents," URL: http://developer.android.com/reference/ android/content/Intent.html, March 2015.

[13] ——, “Manifest," URL: http://developer.android.com/guide/topics/ manifest/manifest-element.html, March 2015.

[14] ——, "Market Share," URL: http://tiny.uzh.ch/cT, March 2015.

[15] ——, "Monitoring the Battery Level and Charging State," URL: http://developer.android.com/training/monitoring-device-state/ battery-monitoring.html, March 2015.

[16] ——, "Source Code," URL: http://grepcode.com/snapshot/repository. grepcode.com/java/ext/com.google.android/android/4.0.4_r2.1/, March 2015.

[17] ——, "Your (My) Way," URL: http://tiny.uzh.ch/wr, March 2015.

[18] Apache Friends, "XAMPP Installers and Downloads for Apache Friends," URL: https://www.apachefriends.org/de/index.html, July 2014.

[19] G. Arena, "Mobile Communication Technologies per Country," URL: http://www.gsmarena.com/network-bands.php3, March 2015.

[20] S. Aroussi, T. Bouabana-Tebibel, A. Mellouk, "Empirical QoE/QoS Correlation Model Based on Multiple Parameters for VoD Flows," IEEE Global Communications Conference (GLOBECOM 2012), California, USA, December 2012, pp. 1963-1968.

[21] L. Ausubel, P. Milgrom, “The Lovely but Lonely Vickrey Auction," Stanford Institute for Economic Policy Research, Discussion Papers 03-036, August 2004.

[22] Backsmali/Smali, "Manager," URL: http://forum.xda-developers.com/ showthread.php?t=2311766, March 2015.

[23] D. Bergemann, J. Vaelimaeki, “The Dynamic Pivot Mechanism," Econometrica, Vol. 78, No. 2, pp. 771-789, March 2010.

[24] D. Bertsekas, Just the Facts1o1: Introduction to Probability, 2nd ed. Cram101, 1 2015, ch. 1, key Terms. 
[25] K. Binmore, D. Harbord, "Bargaining Over Fixed-to-mobile Termination Rates: Countervailing Buyer Power as a Constraint on Monopoly Power," Journal of Competition Law and Economics, Vol. 1, No. 3, pp. 449-472, September 2005.

[26] T. (Brazil), "National Roaming," URL: http://tiny.uzh.ch/ws, March 2015.

[27] P. Casas, M. Seufert, R. Schatz, "YOUQMON: A System for On-line Monitoring of YouTube QoE in Operational 3 G Networks," SIGMETRICS Perform. Eval. Rev., Vol. 41, No. 2, pp. 44-46, August 2013.

[28] R. Cavallo, D. Parkes, S. Singh, "Efficient Mechanisms with Dynamic Populations and Dynamic Types," Unpublished manuscript, Harvard University, 2009.

[29] C. Chun-Lung, "Dual SIM (Subscriber Identity Module) Card Connector: US Patent 6,623,305," Spetmeber 2003.

[30] Cisco, "Quality of Service for Voice over IP," URL: http://www.cisco. $\mathrm{com} / \mathrm{c} / \mathrm{en} / \mathrm{us} / \mathrm{td} / \mathrm{docs} /$ ios/solutions_docs/qos_solutions/QoSVoIP/ QoSVoIP.html, July 2014.

[31] E. Clarke, "Multipart Pricing of Public Goods," Public Choice, Vol. 1 1, No. 1, pp. 17-33, September 1971.

[32] ComCom, "Federal communications commission," URL: http://www. comcom.admin.ch/index.html?lang=en, March 2015.

[33] E. David, R. Azoulay-Schwartz, S. Kraus, "An English Auction Protocol for Multi-attribute Items," Workshop on Agent-Mediated Electronic Commerce (AAMAS 2002), Bologna, Italy, July 2002, pp. 52-68.

[34] R. Dewenter, J. Haucap, "The Effects of Regulation Mobile Termination Rates for Asymmetric Networks," European Journal of Law and Economics, Vol. 20, pp. 185-197, July 2005.

[35] Digital Cellular Telecommunications System (Phase 2+), "Universal Mobile Telecommunications System (UMTS): AT Command Set for User Equipment (UE) (3GPP TS 27.007 version 8.5.0 Release 8)," October 2008.

[36] M. Directory, “MNO Directory," URL: http://www.mnodirectory.com/ europe-volume.html, February 2016. 
[37] Ebay, "Ebay,” URL: http://www.ebay.com/, March 2015.

[38] Eclipse, "IDE," URL: http://www.eclipse.org/, March 2015.

[39] Econstor, “The Regulation of National Roaming," URL: https: //www.econstor.eu/dspace/bitstream/10419/52213/1/672585162.pdf, March 2015.

[40] EETT, "Hellenic Telecommunications and Posts Commission," URL: http: //www.eett.gr/opencms/opencms/EETT_EN/index.html, March 2015.

[41] R. Erdin, "Non-deterministic Voice-traffic Management through Auctions," Master's thesis, University of Zurich, August 2014.

[42] ERICSSON, "ERICSSON Mobility Report: On the Pulse of The Network Society," URL: http://tiny.uzh.ch/uJ, November 2015.

[43] Eurocomms, "MNOs Must Reform Tariffs," URL: http://tiny.uzh.ch/cR, March 2015.

[44] Europa.eu, "Have your Say on Fixed and Mobile Termination Rates," URL: http://tiny.uzh.ch/xg, March 2016.

[45] European Parliament News, "Efficient Mechanisms with Dynamic Populations and Dynamic Types," URL: http://tiny.uzh.ch/uN, 2015.

[46] T. Fadell, “Dynamic Carrier Selection: US Patent 7,885,654," February 2011.

[47] FCC, "Federal Communications Commission," URL: http://www.fcc. gov/, March 2015.

[48] F. C. C. (FCC), "FCC Auctions," URL: http://wireless.fcc.gov/auctions/ default.htm?job=auctions_home, March 2015.

[49] K. Fernando, R. Kooij, D. De Vleeschauwer, K. Brunnstroem, “Techniques for Measuring Quality of Experience," 8th International Conference on Wired/Wireless Internet Communications (WWIC 2010), Lulea, Sweden, June 2010, pp. 216-227.

[50] M. Fiedler, T. Hossfeld, P. Tran-Gia, "A Generic Quantitative Relationship between Quality of Experience and Quality of Service," Network, IEEE, Vol. 24, No. 2, pp. 36-41, March 2010.

[51] Forum, "How to Talk to the Modem With AT Commands," URL: http: //forum.xda-developers.com/showthread.php?t=1471241, March 2015. 
[52] ——, "Using Internal (com.android.internal) and Hidden (@hide) APIs [Part 1, Introduction]," URL: http://tiny.uzh.ch/wt, March 2015.

[53] E. Fulp, M. Ott, D. Reininger, D. Reeves, "Paying for QoS: An Optimal Distributed Algorithm for Pricing Network Resources," Sixth International Workshop on Quality of Service (IWQoS 1998), Napa, California, USA, May 1998, pp. $75-84$.

[54] C. Genakos, T. Valletti, "Evaluating a Decade of Mobile Termination Rate Regulation," The Economic Journal, Vol. 125, No. 586, pp. F3 1-F48, August 2015.

[55] Github, "CyanogenMod/Android Build," URL: https://github.com/ CyanogenMod/android_build/tree/gingerbread/target/product/ security, March 2015.

[56] GoGrid, “Cloud Provider," URL: http://www.gogrid.com/, March 2015.

[57] S. Gonzalez Ruiz, "Designing for the Extremes (Or Why your Average User Doesn't Exist)," URL: http://sugoru.com/2013/07/14/ designing-for-the-extremes/, October 2015.

[58] Google, "Dex2jar," URL: http://code.google.com/p/dex2jar/ downloads/list, March 2015.

[59] ——, “Google adds Auction," URL: http://support.google.com/ adsense/bin/answer.py?hl=en\&answer=160525, March 2015.

[6o] ——, "Smali/Baksmali," URL: https://github.com/JesusFreke/smali, February 2016.

[61] T. Groves, "Incentives in Teams," Econometrica, Vol. 41, No. 4, pp. 617-31, July 1973.

[62] D. Harbord, M. Pagnozzi, "Network-Based Price Discrimination and 'Billand-Keep' vs. 'Cost-Based' Regulation of Mobile Termination Rates," Review of Network Economics, Vol. 9, No. 1, pp. 1-44, March 2010.

[63] F. Hecht, "LiveShift: A Time-Shifted P2P Multimedia Streaming Approach," Ph.D. dissertation, Communication Systems Group, Department of Informatics, University of Zurich, Zurich, Switzerland, December 2013.

[64] T. Hossfeld, L. Skorin-Kapov, P. Heegaard, M. Varela, K. Chen, "On Additive and Multiplicative QoS-QoE Models for Multiple QoS Parameters," PQS 2016 5th ISCA/DEGA Workshop on Perceptual Quality of Systems, 
Berlin, Germany, April 2016, pp. 44-48.

[65] T. Hoßfeld, P. Tran-Gia, M. Fiedler, "Quantification of Quality of Experience for Edge-Based Applications," 2oth International Teletraffic Congress (ITC20 2007), Ottawa, Canada, June 2007, pp. 361-373.

[66] M. Huhns, J. Vidal, “Online Auctions,” Internet Computing, IEEE, Vol. 3, No. 3, pp. 103-105, May 1999.

[67] Hulu, "Hulu Bandwidth Recommendations," URL: http://www.hulu. com/support/article/197541, January 2014.

[68] ITU, “CPP in Europe," URL: http://www.itu.int/osg/spu/ni/fmi/ regulation/Survey2.pdf, March 2015.

[69] ——, "Termination Cost Regulation," URL: http://www.itu.int/net/ itunews/issues/2010/03/20.aspx, March 2015.

[70] ITU-D, “Mobile Termination Rates and Roaming," URL: http://tiny.uzh. $\mathrm{ch} /$ wi, March 2015.

[71] ——, "Mobile Voice Service and Termination Rates," URL: http://tiny. uzh.ch/wj, March 2015.

[72] ——, "Roaming and Mobile Termination Rates," URL: http://tiny.uzh. ch/wk, March 2015.

[73] ITU-T, "Methods for Subjective Determination of Transmission Quality," ITU-T Recommendation P.80o, June 1998.

[74] ——, "End-user Multimedia QoS Categories," ITU-T Recommendation G.1010, November 2001.

[75] _- "One-way Transmission Time," ITU-T Recommendation G.1 14, May 2003.

[76] ——, "The E-model, a Computational Model for Use in Transmission Planning," ITU-T Recommendation G.107, March 2003.

[77] —_, “Mean Opinion Score (MOS) Terminology,” ITU-T Recommendation P.800.1, November 2006.

[78] ——, "Subjective Evaluation of Conversational Quality," ITU-T Recommendation P.805, October 2007.

[79] ——, “E-model Tutorial," URL: http://tiny.uzh.ch/wu, June 2014. 
[80] ——, "Socio-economic Assessment of Future Networks by Tussle Analysis," ITU-T Recommendation Y.3013, August 2014.

[81 ] — , “Transmission Impairments Due to Speech," ITU-T Recommendation G.113, July 2014.

[82] ——, "International Telecommunication Union - Telecommunication Standardization Sector (ITU-T)," URL: http://www.itu.int/en/ITU-T, March 2015.

[83] Joyent, “Node.js," URL: http://nodejs.org/about/, July 2014.

[84] A. Jurgelionis, J. Laulajainen, M. Hirvonen, A. Wang, "An Empirical Study of NetEm Network Emulation Functionalities," 2oth International Conference on Computer Communications and Networks (ICCCN 2011), Maui, Hawaii, July 201 1, pp. 1-6.

[85] M. Kaku, Physics of the Future: How Science Will Shape Human Destiny and Our Daily Lives by the Year 210o. Doubleday, March 2011.

[86] K. Kazuhiko Takeno, M. Ichimura, K. Takano, J. Yamaki, "Influence of Cycle Capacity Deterioration and Storage Capacity Deterioration on Li-ion Batteries Used in Mobile Phones," Journal of Power Sources, Vol. 142, No. 1-2, pp. 298-305, March 2005.

[87] Keloo Network, "1000o General Knowledge Questions and Answers," URL: http://www.keloo.ro/doc/1000o_intrebari.pdf, July 2014.

[88] K. Knopper, “Knoppnix - Live Linux Filesystem on CD,” URL: http:// www.knopper.net/knoppix/index-en.html, July 2014.

[89] J. Kuster, E. Huber, R. Lippmann, A. Schmid, E. Schneider, U. Witschi, R. Wuest, Handbuch Projektmanagement. Springer Berlin Heidelberg, 2008.

[90] K. Laghari, K. Connelly, "Toward Total Quality of Experience: A QoE Model in a Communication Ecosystem," Communications Magazine, IEEE, Vol. 50, No. 4, pp. 58-65, April 2012.

[91] S. Liniger, "Implementation of an Automatic, On-demand Mobile Network Operator (MNO) Selection Mechanism on Android Devices," August 2013, bachelor thesis, University of Zurich, Zurich, Switzerland.

[92] Linux Foundation, "netem | The Linux Foundation," URL: http://www. linuxfoundation.org/collaborate/workgroups/networking/netem, July 
2014.

[93] S. Littlechild, "Mobile Termination Charges: Calling Party Pays versus Receiving Party Pays," Telecommunications Policy, Vol. 30, No. 5-6, pp. 242277, June 2006.

[94] N. P. Lookup, “NLS Cost,” URL: http://tiny.uzh.ch/wm, March 2015.

[95] M-line Telecoms, “Minimum Call Charge Trick,” URL: http://tiny.uzh. ch/wf, February 2016.

[96] G. Machado, B. Stiller, "Investigations of an SLA Support System for Cloud Computing (SLACC)," Praxis der Informationsverarbeitung und Kommunikation (PIK), Vol. 34, No. 2, pp. 80-86, June 2011.

[97] R. P. McAfee, J. McMillan, "Auctions and Bidding," Journal of Economic Literature, Vol. 25, No. 2, pp. 699-738, June 1987.

[98] Mediafone, “Mobile Number Portability Registry Provider," URL: http: //www.mediafon.eu/, December 2015.

[99] R. Mok, E. Chan, R. Chang, "Measuring the Quality of Experience of HTTP Video Streaming," IFIP/IEEE International Symposium on Integrated Network Management (IM 2011), Dublin, Ireland, May 201 1, pp. 485-492.

[100] Motorola, "Realistic LTE Performance - From Peak Rate to Subscriber Experience," URL: http://tiny.uzh.ch/wn, February 2016.

[101] Nielsen, "Average U.S. Smartphone Data Usage up 89\% as Cost per MB Goes Down 46\%," URL: http://tiny.uzh.ch/cS, March 2015.

[102] - - "Smartphone Penetration Rate by Age Group," URL: http://tiny. uzh.ch/wp, March 2015.

[103] - -, “Smartphones Penetration,” URL: http://tiny.uzh.ch/wo, March 2015 .

[104] Novascola, “Quizfragen und Antworten,” URL: http://www.novascola. ch/quizfragen-und-antworten, July 2014.

[105] A. Ockenfels, D. Reiley, A. Sadrieh, "Online Auctions," National Bureau of Economic Research, Working Paper 12785, December 2006.

[106] OFCOM, "Federal Office of Communications," URL: http://www. bakom.admin.ch/, May 2015. 
[107] Opus, "Voice Coding with Opus," URL: http://www.opus-codec.org/ presentations/opus_voice_aes135.pdf, July 2014.

[108] Oracle, "Java Reflection," URL: http://docs.oracle.com/javase/tutorial/ reflect/, March 2015.

[109] K. Pahlavan, P. Krishnamurthy, Principles of Wireless Networks. Prentice Hall PTR, 2002, ch. 7, paragraph 7.3.

[1 10] H. Park, "Method for Verifying Personalization in Mobile Radio Terminal: US Patent 6,138,005," October 2000.

[111] Pay Per Lough, "Pay-per-laugh: The Comedy Club that Charges Punters Having Fun," URL: http://tiny.uzh.ch/nH, July 2015.

[112] P. Poullie, C. Schmitt, B. Stiller, "Designing Future Networks: The Investigation of Socio-economic Awareness by the Tussle Analysis," IEEE Conference on Standards for Communications and Networking (CSCN 2015), Tokyo, Japan, October 2015, pp. 19-24.

[113] PrePaidGSM, “TDC Voice Tariffs,” URL: http://tiny.uzh.ch/we, February 2016.

[114] Rackspace, “Cloud Provider," URL: http://www.rackspace.com/, March 2015.

[115] P. Reichl, S. Egger, S. Moeller, K. Kilkki, M. Fiedler, T. Hossfeld, C. Tsiaras, A. Asrese, "Towards a Comprehensive Framework for QoE and User Behavior Modelling," 7th International Workshop on Multimedia Experience (QoMEX 2015), Pylos-Nestoras, Greece, May 2015, pp. 1-6.

[116] P. Reichl, S. Egger, R. Schatz, A. D'Alconzo, "The Logarithmic Nature of QoE and the Role of the Weber-Fechner Law in QoE Assessment," IEEE International Conference on Communications (ICC 2010), Cape Town, South Africa, May 2010, pp. 1-5.

[117] P. Reichl, F. Hammer, "Charging for Quality-of-Experience: A New Paradigm for Pricing IP-based Service," 2nd ISCA/DEGA Tutorial and Research Workshop on Perceptual Quality of Systems (PQS 2006), Berlin, Germany, September 2006, pp. 1-8.

[118] P. Reichl, F. Hammer, M. Balinova, "Conversational Interactivity in Slowmotion: A Comparison of Phone Dialogues and Opera Scenes," Proc. 2nd International Conference on Sound and Music Computing (SMC 2005), Salerno, Italy, November 2005, pp. 1-11. 
[1 19] P. Reichl, P. Maillé, P. Zwickl, A. Sackl, "On the Fixpoint Problem of QoEbased Charging," 6th International Conference on Performance Evaluation Methodologies and Tools (ValueTools 2012), Cargèse, France, October 2012 , pp. 235-242.

[120] A. Richards, M. Antoniades, V. Witana, G. Rogers, "Mapping User Level QoS from a Single Parameter," Second International Conference on Multimedia Networks and Services (MMNS 1998), Versailles, France, November 1998, pp. 1-22.

[121] T. Rockoff, M. Groves, "Design of an Internet-based System for Remote Dutch Auctions," Internet Research, Vol. 5, No. 4, pp. 10-16, December 1995 .

[122] M. Rösch, "Quality-of-Experience Measurement Setup," August 2014, bachelor thesis, University of Zurich, Zurich, Switzerland.

[123] M. Said, Auctions with Dynamic Populations: Efficiency and Revenue Maximization. Auctions, Market Mechanisms and Their Applications. Springer, 2009, pp. 87-88.

[124] J. Schiller, Mobile Communications, 2nd ed. Addison-Wesley, 2003, ch. 1.2.

[125] ——, Mobile Communications, 2nd ed. Addison-Wesley, 2003, ch. 4, p. 114 .

[126] J. Seigneur, X. Titi, T. El Maliki, “Towards Mobile/Wearable Device Electrosmog Reduction Through Careful Network Selection,” 1 st Augmented Human International Conference (AH 2010), Megève, France, April 2010, pp. $1-5$.

[127] I. Sesia, S. Toufik, M. Baker, Front Matter. John Wiley \& Sons, Ltd, 2009, pp. i-xxxv.

[128] Stackoverflow, "Can a telephony.Phone Object Be Instantiated Through the SDK?” URL: http://tiny.uzh.ch/cQ, March 2015.

[129] V. T., "Analysis of Modem Integration in Open Source Smartphone Platforms," Master's thesis, Oulu University of Applied Sciences, Oulu, Finland, March 2011.

[130] TATA, “WANem: The Wide Area Network Emulator," URL: http:// wanem.sourceforge.net/, July 2014. 
[131] H. Te-Yuan, H. Polly, C. Kuan-Ta, W. Po-Jung, "Could Skype Be More Satisfying? A QoE-centric Study of the FEC Mechanism in an Internet-scale VoIP System,” Network, IEEE, Vol. 24, No. 2, pp. 42-48, March 2010.

[132] Telcordia Technologies, "Mobile Number Portability Registry Provider," URL: http://iconectiv.com/, December 2015.

[133] TRAI, “Telecom Regulatory Authority of India," URL: http://www.trai. gov.in/, March 2015.

[134] C. Tsiaras, “Survey,” URL: http://tiny.uzh.ch/wq, March 2015.

[135] C. Tsiaras, S. Liniger, B. Stiller, "An Automatic and On-demand MNO Selection Mechanism," IEEE/IFIP Network Operations and Management Symposium (NOMS 2014), Krakow, Poland, May 2014, pp. 1-8.

[136] _-, "Automatic and On-demand Mobile Network Operator (MNO) Selection Mechanism Demonstration," IEEE/IFIP Network Operations and Management Symposium (NOMS 2014), Krakow, Poland, May 2014, pp. 1-2.

[137] C. Tsiaras, M. Rösch, B. Stiller, "VoIP-based Calibration of the DQX Model," IFIP Networking 2015, Toulouse, France, May 2015, pp. 1-9.

[138] C. Tsiaras, A. Sehgal, S. Seeber, D. Doenni, B. Stiller, J. Schönwälder, G. Rodosek, “Towards Evaluating Type of Service Related Quality-ofExperience on Mobile Networks," 7th IFIP Wireless and Mobile Networking Conference (WMNC 2014), Vilamoura, Portugal, May 2014, pp. 1-8.

[139] C. Tsiaras, B. Stiller, "Challenging the Monopoly of Mobile Termination Charges with an Auction-Based Charging and User-Centric System (AbaCUS)," Conference on Networked Systems (NetSys 2013), Stuttgart, Germany, March 2013, pp. 110-117.

[140] ——, "A Deterministic QoE Formalization of User Satisfaction Demands (DQX)," 39th IEEE Conference on Local Computer Networks (LCN 2014), Edmonton, Canada, September 2014, pp. 227-235.

[141] J. Uberti, S. Dutton, "WebRTC Plugin-free Realtime Communication," URL: http://io13webrtc.appspot.com/, July 2014.

[142] M. Ulrike, L. Young Han, “The Bidder's Curse," American Economic Review, Vol. 101, No. 2, pp. 749-87, April 2011. 
[143] E. Utherland, "International Roaming Charges: Over-charging and Competition Law," Telecommunications Policy, Vol. 25, No. 1-2, pp. 5-20, December 2001.

[144] M. Venkataraman, M. Chatterjee, C. Siddhartha, "Evaluating Quality of Experience for Streaming Video in Real Time," IEEE Global Telecommunications Conference (GLOBECOM 2009), Honolulu, Hawaii, USA, November 2009, pp. 1-6.

[145] W. Vickrey, "Counterspeculation, Auctions, and Competitive Sealed Tenders," The Journal of Finance, Vol. 16, No. 1, pp. 8-37, March 1961.

[146] W3 C, "WebRTC 1.0: Real-time Communication between Browsers," URL: http://dev.w3.org/2011/webrtc/editor/webrtc.html, July 2014.

[147] S. Wahlmueller, P. Zwickl, P. Reichl, "Pricing and Regulating Quality of Experience," 8th EURO-NGI Conference on Next Generation Internet (NGI 2012), Karlskrona, Sweden, June 2012, pp. 57-64.

[148] WebRTC, “Opus in Low Bandwidth Conditions," URL: https://code. google.com/p/webrtc/issues/detail?id=2025, July 2014.

[149] Wikipedia, "Apple SIM Card," URL: http://en.wikipedia.org/wiki/ Apple_SIM, January 2014.

[150] ——, “Dual-SIM,” URL: http://en.wikipedia.org/wiki/Dual_SIM, March 2015. 


\section{Other Author Publications}

\section{Journal Article}

- A. Tasiopoulos, C. Tsiaras, and S. Toumpis: Optimal and Achievable Cost/delay Tradeoffs in Delay-tolerant Networks, Elsevier, Computer Networks, Vol. 70, No. 9, September 2014, pp 59-74. URL: http://dx.doi.org/ 10.1016/j.comnet.2014.05.006.

\section{Conference Papers}

- T. Bocek, C. Killer, C. Tsiaras, and B. Stiller: An NFC Relay Attack with Off-the-shelf Hardware and Software, 1oth International Conference on Autonomous Infrastructure, Management and Security (AIMS 2016), Munich, Germany, June 2016, pp 1-13.

- C. Tsiaras, L. Hobi, F. Hofstetter, S. Liniger, and B. Stiller: parkITsmart: Minimization of Cruising for Parking, 24th International Conference on Computer Communications and Networks (ICCCN 2015), Las Vegas, Nevada, USA, August 2015, pp 1-8. URL: http://dx.doi.org/10.1109/ICCCN. 2015.7288448 .

- D. Dönni, G. Machado, C. Tsiaras, and B. Stiller: Schengen Routing: A Compliance Analysis, 9th International Conference on Autonomous Infrastructure, Management and Security (AIMS 2015), "AIMS 2015", Gent, Belgium, May 2015, pp 1-12. URL: http://www.springer.com/us/book/ 9783319200330\#.

- A. Tasiopoulos, C. Tsiaras, and S. Toumpis: On the Cost/Delay Tradeoff of Wireless Delay Tolerant Geographic Routing, IEEE International Symposium (WoWMoM 2012), "World of Wireless, Mobile and Multimedia Networks”, San Francisco, California, U.S.A., June 2012, pp 1-9. URL: http://dx.doi.org/10.1109/WoWMoM.2012.6263706.

- C. Tsiaras, M. Waldburger, G. Machado, A. Vancea, and B. Stiller: The Design of a Single Funding Point Charging Architecture, 18th EUNICE Conference on Information and Communications Technologies, "Information 
and Communication Technologies”, Budapest, Hungary, August 2012, pp 148-160. URL: http://link.springer.com/chapter/10.1007\%2F978-3-642-32808-4_ 14 .

\section{Technical Reports}

- B. Stiller, C. Tsiaras, A. Lareida, L. Kristiana, R. Garg, D. Dönni, and C. Schmitt: Communication Systems VIII; IFI, IFI-Technical Report No. IFI2015.02, Zürich, Switzerland, June 2015, pp 1-117. URL: http://www.csg. uzh.ch/teaching/fs15/comsys/extern/IFI-2015.02.pdf.

- B. Stiller, D. Dönni, F. Hecht, A. Lareida, G. Machado, C. Tsiaras, A. Vancea, and M. Waldburger: Internet Economics VII; IFI Technical Report 2013.01, Zürich, Switzerland, April 2013, pp 1-191. URL: http://www.csg. uzh.ch/teaching/hs12/inteco/extern/IFI-2013.01.pdf.

- B. Stiller, K. Farkas, F. Hecht, G. Machado, P. Poullie, F. Santos, C. Tsiaras, A. Vancea, and M. Waldburger: Internet Economics VI; IFI Technical Report, No. IFI-2012.02, April 2012, pp 1-147. URL: http://www.csg.uzh.ch/teaching/ hs11/inteco/extern/IFI-2012.02.pdf. 


\section{Appendix}

\section{A.1 Formal Proofs of AQX Equations}

This appendix displays complete formal proofs of AQX equations from Chapter 3.

Equation 3.10.

$$
\begin{aligned}
& e_{a}\left(x_{\circ}\right):=e_{\circ} \\
\Rightarrow & e_{\circ}=h \cdot e^{-\lambda \cdot x_{\circ}^{m}}+\mu \\
\Leftrightarrow & \frac{e_{\circ}-\mu}{h}=e^{-\lambda \cdot x_{\circ}^{m}} \\
\Leftrightarrow & \ln \left(\frac{e_{\circ}-\mu}{h}\right)=-\lambda \cdot x_{\circ}^{m} \\
\Leftrightarrow & \lambda=x_{\circ}^{-m} \ln \left(\frac{h}{e_{\circ}-\mu}\right)
\end{aligned}
$$

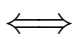

$\Longleftrightarrow$

Equation 3.15.

$$
\begin{aligned}
& e_{i}\left(x_{\circ}-\delta^{\prime}\right)=e_{\circ}-\varepsilon^{\prime} \\
& \Leftrightarrow h \cdot\left(1-e^{-\lambda \cdot\left(x_{0}-\delta^{\prime}\right)^{m^{-}}}\right)+\mu=e_{\circ}-\varepsilon^{\prime} \\
& \Leftrightarrow 1-e^{-\lambda \cdot\left(x_{0}-\delta^{\prime}\right)^{m^{-}}}=\frac{e_{\mathrm{o}}-\mu-\varepsilon^{\prime}}{h} \\
& \Leftrightarrow e^{-\lambda \cdot\left(x_{0}-\delta^{\prime}\right)^{m^{-}}}=\frac{h-e_{0}+\mu+\varepsilon^{\prime}}{h} \\
& \Leftrightarrow e^{-\left(\frac{x_{0}-\delta^{\prime}}{x_{0}}\right)^{m^{-}} \cdot \ln \left(\frac{h}{h-e_{0}+\mu}\right)}=\frac{h-e_{0}+\mu+\varepsilon^{\prime}}{h} \\
& \Leftrightarrow e^{\ln \left(\frac{h-e_{0}+\mu}{h}\right)\left(\frac{x_{0}-\delta^{\prime}}{x_{0}}\right)^{m^{-}}}=\frac{M-e_{0}+\varepsilon^{\prime}}{h}
\end{aligned}
$$
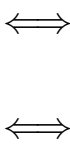


$$
\begin{aligned}
& \Leftrightarrow \frac{h-e_{0}+\mu}{h}\left(\frac{x_{0}-\delta^{\prime}}{x_{0}}\right)^{m^{-}}=\frac{M-e_{\circ}+\varepsilon^{\prime}}{h} \\
& \Leftrightarrow \frac{M-e_{0}}{h}\left(\frac{x_{0}-\delta^{\prime}}{x_{0}}\right)^{m^{-}}=\frac{M-e_{0}+\varepsilon^{\prime}}{h} \\
& \Leftrightarrow\left(\frac{x_{\circ}-\delta^{\prime}}{x_{\circ}}\right)^{m^{-}}=\log _{\left(\frac{M-e_{0}}{h}\right)}\left(\frac{M-e_{\circ}+\varepsilon^{\prime}}{h}\right) \\
& \Leftrightarrow m^{-}=\log \left(\frac{x_{0}-\delta^{\prime}}{x_{0}}\right)\left[\log \left(\frac{M-e_{0}}{h}\right)\left(\frac{M-e_{0}+\varepsilon^{\prime}}{h}\right)\right] \\
& \Leftrightarrow m^{-}=\frac{\ln \left(\frac{\ln \frac{M-e_{0}+\varepsilon^{\prime}}{h}}{\ln \frac{M-e_{0}}{h}}\right)}{\ln \frac{x_{0}-\delta^{\prime}}{x_{0}}}
\end{aligned}
$$

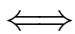

Equation 3.19.

$$
\begin{aligned}
& e_{a}\left(x_{\circ}+\delta\right)=e_{\circ}-\varepsilon^{\prime} \\
& \Leftrightarrow h \cdot e^{-\lambda \cdot\left(x_{0}+\delta\right)^{m^{+}}}+\mu=e_{0}-\varepsilon^{\prime} \\
& \Leftrightarrow e^{-\lambda \cdot\left(x_{0}+\delta\right)^{m^{+}}}=\frac{e_{\circ}-\mu-\varepsilon^{\prime}}{h} \\
& \Leftrightarrow e^{-\left(\frac{x_{0}+\delta}{x_{0}}\right)^{m^{+}} \cdot \ln \left(\frac{h}{e_{0}-\mu}\right)}=\frac{e_{0}-\mu-\varepsilon^{\prime}}{h} \\
& \Leftrightarrow e^{\ln \left(\frac{e_{0}-\mu}{h}\right)^{\left(\frac{x_{0}+\delta}{x_{0}}\right)^{m^{+}}}}=\frac{e_{0}-\mu-\varepsilon^{\prime}}{h} \\
& \Leftrightarrow\left(\frac{e_{\circ}-\mu}{h}\right)^{\left(\frac{x_{0}+\delta}{x_{0}}\right)^{m^{+}}}=\frac{e_{\circ}-\mu-\varepsilon^{\prime}}{h} \\
& \Leftrightarrow\left(\frac{x_{\circ}+\delta}{x_{\circ}}\right)^{m^{+}}=\log _{\left(\frac{e_{0}-\mu}{h}\right)}\left(\frac{e_{\circ}-\mu-\varepsilon^{\prime}}{h}\right) \\
& \Leftrightarrow m^{+}=\log _{\left(\frac{x_{0}+\delta}{x_{0}}\right)}\left[\log _{\left(\frac{e_{0}-\mu}{h}\right)}\left(\frac{e_{0}-\mu-\varepsilon^{\prime}}{h}\right)\right]
\end{aligned}
$$
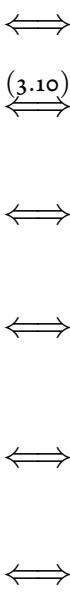

Equation 3.21.

$$
e_{a}\left(x_{\circ}-\delta^{\prime}\right)=e_{\circ}+\varepsilon
$$




$$
\begin{aligned}
& \Leftrightarrow h \cdot e^{-\lambda \cdot\left(x_{\circ}-\delta^{\prime}\right)^{m^{-}}}+\mu=e_{\circ}+\varepsilon \\
& \Leftrightarrow e^{-\lambda \cdot\left(x_{\circ}-\delta^{\prime}\right)^{m^{-}}}=\frac{e_{\circ}-\mu+\varepsilon}{h} \\
& \Leftrightarrow e^{-\left(\frac{x_{0}-\delta^{\prime}}{x_{\circ}}\right)^{m^{-}} \cdot \ln \left(\frac{h}{e_{0}-\mu}\right)}=\frac{e_{\circ}-\mu+\varepsilon}{h} \\
& \Leftrightarrow e^{\ln \left(\frac{e_{0}-\mu}{h}\right)}\left(\frac{x_{0}-\delta^{\prime}}{x_{\circ}}\right)^{m^{-}}=\frac{e_{\circ}-\mu+\varepsilon}{h} \\
& \Leftrightarrow\left(\frac{e_{\circ}-\mu}{h}\right)^{\left(\frac{x_{0}-\delta^{\prime}}{x_{\circ}}\right)^{m^{-}}}=\frac{e_{\circ}-\mu+\varepsilon}{h} \\
& \Leftrightarrow\left(\frac{x_{\circ}-\delta^{\prime}}{x_{\circ}}\right)^{m^{-}}=\log _{\left(\frac{e_{0}-\mu}{h}\right)}\left(\frac{e_{\circ}-\mu+\varepsilon}{h}\right) \\
& \Leftrightarrow m^{-}=\log _{\left(\frac{x_{0}-\delta^{\prime}}{x_{0}}\right)}\left[\log _{\left(\frac{e_{0}-\mu}{h}\right)}\left(\frac{e_{\circ}-\mu+\varepsilon}{h}\right)\right] \\
& \Leftrightarrow m^{-}=\frac{\ln \left(\frac{\ln \frac{e_{0}-\mu+\varepsilon}{h}}{\ln \frac{e_{0}-\mu}{h}}\right)}{\ln \frac{x_{0}-\delta^{\prime}}{x_{\circ}}}
\end{aligned}
$$

Equation 3.24.

$$
\begin{aligned}
\frac{(3.22)}{(3.23)} & \Rightarrow\left(\frac{x_{1}}{x_{2}}\right)^{m_{a, x}}=\frac{\ln \frac{e_{a, 1}-\mu}{h}}{\ln \frac{e_{a, 2}-\mu}{h}} \\
& \Leftrightarrow m_{a, x}=\log _{\left(\frac{x_{1}}{x_{2}}\right)\left[\frac{\ln \frac{e_{a, 1}-\mu}{h}}{\ln \frac{e_{a, 2}-\mu}{h}}\right]} \\
& \Leftrightarrow m_{a, x}=\frac{\ln \left(\frac{\ln \frac{e_{a, 1}-\mu}{h}}{\ln \frac{e_{a, 2}-\mu}{h}}\right)}{\ln \frac{x_{1}}{x_{2}}}
\end{aligned}
$$




\section{List of Figures}

1.1 The MTR Ecosystem . . . . . . . . . . . . . . 2

1.2 Thesis Overview . . . . . . . . . . . . 4

2.1 Related Work Overview _. . . . . . . . . . . . 12

2.2 QoE Mapping Function of Packet Loss Ratio in the IQX Hypothesis [50] ..................... 22

2.3 Reference Connection of the E-Model [76] . . . . . . 23

2.4 SLACC Solution Overview [96] . . . . . . . . . 26

3.1 MOS Evolution for $\operatorname{IV}\left(e_{i}\right)$ and $\operatorname{AV}\left(e_{a}\right) \ldots \ldots 33$

3.2 Plot for Different $m$ Values of Equation $3.8 \ldots \ldots$. . . . . 37

3.3 Plot for Different $m$ Values of Equation $3.11 \ldots \ldots$

3.4 MOS Change for IV . . . . . . . . . . . . . . 39

3.5 MOS Change for AV . . . . . . . . . . . 42

3.6 Generic MOS Evolution for Equally Participating IV $(m=1)$ and $\mathrm{AV}(m=3) \ldots \ldots \ldots . \ldots . \ldots 45$

$4.1 \quad$ Experimental Setup . . . . . . . . . . . . . . . 52

4.2 AQX vs Emodel (Latency) . . . . . . . . . . . . . . 59

4.3 AQX vs Emodel (Latency - Updated $m^{+}$) . . . . . . . 61

4.4 AQX vs Emodel and IQX (Packet Loss) . . . . . . . . . . 62

4.5 AQX MOS (Bandwidth) . . . . . . . . . . . 63

4.6 3D-Graph of the AQX Model for Multiple Variables . . . . 65

5.1 Stakeholders of the Internet Ecosystem [ 112$] \ldots 70$

5.2 MTR Stakeholders Map . . . . . . . . . . . . 73

5.3 Key Elements of AbaCUS . . . . . . . . . . . 90

5.4 The AbaCUS MvNO Selection Algorithm Diagram . . . . 92

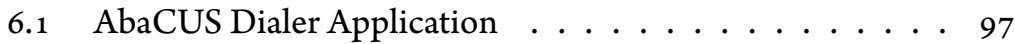

6.2 MNO Selection with AT Commands . . . . . . . . . . . 99

6.3 MNO Selection Mechanism Implementation Steps . . . 100 
6.4 Evaluation Set-up . . . . . . . . . . . . . . . 105

6.5 Switching Time Between MNOs . . . . . . . . . . . 107

6.6 Min and Max Values for the MNO Switching Time . . . . 108

6.7 MNO Switching Time and Signal Strength Correlation . . 109

6.8 MNO Switching Time During the Day . . . . . . . . 110

6.9 MNO Switching Time $>10 \mathrm{~s} \ldots \ldots \ldots 110$

6.10 AbaCUS Service Request . . . . . . . . . . . 112

6.11 AbaCUS E2E MNO Calling-time ........... 114 


\section{List of Tables}

2.1 The MOS Scheme Recommended by the ITU-T [73 ] . . 20

2.2 QoE Models ................. 21

3.1 IV and AV Examples ............. 31

4.1 $x_{0}, x_{\min }, x_{\max }$ and References Used for the Evaluation . . . 57

4.2 Influence Factors Results of the Single Variable Scenarios . 58

4.3 Updated Influence Factors Results of the Single Variable Scenarios ................ . 60

4.4 Collected MOS for Mixed Variables Compared to the Calculated MOS . . . . . . . . . . . . . 64

5.1 MTR Ecosystem Stakeholders . . . . . . . . . 72

5.2 Users's Behavior Survey Results . . . . . . . . . . 73

5.3 QoE-related Variables Identification of Mobile Calls . . . . 77

5.4 QoE-related Variables Classification of Mobile Calls . . . . 78

5.5 Min-Max QoE-related Variables Identification of Mobile Calls . . . . . . . . . . . . . . . . . 79

5.6 Desired Variables Values Identification of Mobile Calls . . 80

5.7 Influence Factor $\left(\mathrm{m}^{-} / \mathrm{m}^{+}\right)$Calculation (for $\left.a, b \in(0,100)\right) \quad 82$

5.8 Influence Factor $(m)$ Evolution for IV . . . . . . 83

5.9 Influence Factor $(m)$ Evolution for AV . . . . . . . 84

5.10 Importance Factors Example for the Mobile Calls Service . 85

5.11 Caller's Preferences Example . . . . . . . . . . . 87

5.12 Two MvNOs Mobile Call Offers Example . . . . . . . 88

5.13 QoS-Cs List. . . . . . . . . . . . . . . . . . . 88

5.14 Caller's Preferences Request Example . . . . . . . . . 89

5.15 MvNO Mobile Call Offers Example . . . . . . . . . 91

6.1 Signal Strength Values . . . . . . . . . 106

6.2 Mobile Device Characteristics . . . . . . . . . . 112

6.3 Power Consumption Evaluation ........... 112 


\section{Acknowledgments}

I am very grateful to a number of people that have helped me, directly or indirectly, through the arduous work that culminates with this thesis.

Firstly, I would like to thank Prof. Dr. Burkhard Stiller for believing in me, allowing me to join the Communication Systems Group (CSG) at the University of Zurich, giving me freedom to research topics that I found interesting, and precious help, feedback, and understanding during several difficult stages. In addition, I would like to thank Prof. Dr. Peter Reichl for being my co-supervisor introducing me to the very interesting topic of Quality-of-Experience ( $\mathrm{OoE}$ ) and inspiring me, as well as providing me with highly valuable feedback. Thank you!

I would also like to warmly thank "my" co-authors, Tobias Hoßfeld, Samuel Liniger, Manuel Rüsch, Sebastian Seeber, and Anuj Sehgal, for coauthoring key scientific papers together. You all have greatly contributed to both my work and my overall development as a scientist. I am particularly grateful to Patrick Poullie for providing the German translation to this thesis' abstract. Furthermore, I would like to thank all 14 assignment, diploma, bachelor, and master students that I have supervised while working on this thesis for the rich discussions and contributions.

It has been an immense pleasure working at the CSG and enjoying the true friendship of my work colleagues, including warm discussions about diverse subjects with current colleagues Dr. Thomas Bocek, Andri Lareida, and Patrick Poullie, as well as former colleagues Prof. Dr. Karoly Farkas, Dr. Fabio Hecht, Dr. Guilherme Sperb Machado, Dr. Oleksiy Mazhelis, Dr. Flavio Santos, Dr. Andrei Vancea, and Dr. Martin Waldburger.

Special thanks to IFI's administration staff Andreas Corti, Marina Josipovic, Barbara Jost, and Miriam Plichta, and to systems administrators Hanspeter Kunz, Beat Rageth, and Enrico Solcà for ensuring a smooth working environment and for providing prompt solutions on every request.

I would like to thank all my former professors for helping me to gain the knowledge that helped me to tackle the problems that I challenged dur- 
ing my Ph.D. Special thanks to Prof. Dr. Christos Chaldoupis, Prof. Dr. Nikolaos Kylafis, and Prof. Dr. Stavros Toumpis for being an inspiration, believing in me and supporting me when needed.

Also, special thanks to my wife Eleni Vlachaki and close friends Ioannis Antoniou, Apostolos Belokas, Nikos Chatzidimitriou, Spiros Giannatos, Dimitris Gkounis, Dimitris Deligiannis, Vasileios Kappatos, Stavros Karagiannopoulos, Ioannis Katsandredakis, Dr. Konstantinos Koutrolikos, Paris Latsaras, Magda Manassi, Dr. Athanasios Mesemanolis, Thenia Panagou, Nikos Planas, Vivi Spiliopoulou, Chara Vareli, Eleni Veletsanou, Giorgos Vlachakis, Panagiotis Vrakatselis, and Stefanos Petrakis for their support. Finally, I would like to thank my parents for the unconditional support all those years supporting me and providing every possible help. I want to make you proud, like I am proud to be your son. I love you all! 


\section{Curriculum Vitae}

Christos Tsiaras was born on June 3, 1981, in Athens, Greece. Christos holds a four-year degree named "Ptychio" - equivalent to a Swiss Diplom in Physics from the University of Crete (UOC). After his studies in Physics Christos received the Probationary Reserved Officer (PRO) degree from the school of Signal Corps of the Hellenic Armed Forces in Greece. Christos served as an officer for 2 years in the Hellenic Armed Forces and was dismissed with the rank of the $2^{\text {nd }}$ Lieutenant. After that Christos continued his studies at the master program of the department of Computer Science at the Athens University of Economics and Business (AUEB) and received his M.Sc. with the highest distinction. His thesis was entitled "Efficient Minimization of routing cost in Delay Tolerant Networks (DTNs)”, in which a low complexity routing protocol for DTNs has been designed. After his studies, Christos has worked as a Physics teacher in high schools, a freelancer programmer and project manager, starting his own company in Heraklion in 2006.

By mid-2009, Christos Tsiaras has moved to Copenhagen, Denmark to become a software engineer. However, Christos' dream is to work in research and it was time for him to chase it. Thus, by mid-2011 Christos has moved to Zurich, Switzerland to become a doctoral student until mid-2015, as well as a research assistant in the Communication Systems Group at the Department of Informatics of the University of Zurich. The work has involved a multitude of tasks, including wireless and mobile communications and Quality-of-Experience ( $\mathrm{QOE}$ ) modeling, managing the web-page infrastructure, and designing a parking management and monitoring system that aims to minimize the cruising for parking effect in large cities. Christos has been involved in the following research projects: "ParkITsmart (pITs)", "Coinblesk", "ICT COST Action IC 1304: Autonomous Control for a Reliable Internet of Services (ACROSS)", "Auction-based Charging and User-centric System (AbaCUS)", "Socially-aware Management of New Overlay Application Traffic combined with Energy Efficiency in the Inter- 
net (SmartenIT)", "Management of the Future Internet (FLAMINGO)", "Accounting and Monitoring of AAI Services (AMAAIS)", and "Socioeconomic Services for European Research Projects (SESERV)".

Christos' main research interests are Quality-of-Experience (QoE), wireless and mobile communication, and Internet of Things (IoT). His doctoral thesis was supervised by Prof. Dr. Burkhard Stiller (University of Zurich, Switzerland) and Prof. Dr. Peter Reichl (Universität Wien, Austria). 


\section{Glossary}

3GPP $3^{\text {rd }}$ Generation Partnership Project.

AbaCUS Auction-based Charging User-centric System.

ADT Android Developer Tools.

API Application Programming Interface.

AQX Axiomatic Quality-of-Experience Model.

AT Attention (Commands).

$\mathbf{A u}^{2}$ Auction Authority.

AV Antitonic Variable.

BS Base Station.

CDR Call Data Record.

CP Cloud Provider.

CPP Calling Party Pays.

CPU Central Processing Unit.

CSG Communication Systems Group.

DPV Dynamic Pivot Mechanism.

DSM Digital Single Market.

E2E end-to-end.

EC European Commission. 
EC2 Elastic Compute Cloud.

eMOS Expected Mean Opinion Score.

EU European Union.

$\mathbf{e V}^{\mathbf{2}}$ Expected Variable Value.

FCFS First Come First Served.

FNO Fixed Network Operator.

GSM Global System for Mobile Communications.

HD High Definition.

HLR Home Location Register.

IDE Integrated Development Environment.

iLBC Internet Low Bitrate Codec.

IP Internet Protocol.

IQX Interdependency of the Quality-of-Experience and Quality-ofService.

ISP Internet Service Provider.

IT Information Technology.

ITU International Telecommunication Union.

ITU-T International Telecommunication Union Telecommunication Standardization Sector.

IV Isotonic Variable.

LAN Local Area Network.

LTE Long Term Evolution. 
M2M mobile-to-mobile.

MAC Media Access Control.

MCC Mobile Country Code.

MIMO multiple-input and multiple-output.

MNC Mobile Network Code.

MNO Mobile Network Operator.

MNP Mobile Number Portability.

MOS Mean Opinion Score.

MSISDN Mobile Subscriber Integrated Services Digital NetworkNumber.

MTR Mobile Termination Rates.

MVNO Mobile Virtual Network Operator.

MvNO Mobile (Virtual) Network Operator.

NatRoam National Roaming.

NLS Number Lookup Service.

OFCOM Federal Office of Communications.

OLR Overall Loudness Rating.

OS Operating System.

OTT over-the-top.

P2P Peer-to-Peer.

PA Power Amplifier.

QoE Quality-of-Experience. 
QoS Quality-of-Service.

QoS-C Quality-of-Service Class.

RAM Random Access Memory.

RF Radio Frequency.

ROM Read Only Memory.

RPP Receiving Party Pays.

RSSI Received Signal Strength Indication.

SD Standard Definition.

SDK Software Development Kit.

SGS2 Samsung Galaxy S II.

SIM Subscriber Identity Module.

SLA Service-level Agreement.

SLACC Service Level Agreement Support System for Cloud Computing.

SMS Short Message Service.

SP Service Provider.

SS $_{7}$ Signaling System No. 7.

SSH Secure Shell.

STMR Side Tone Masking Rating.

TeR-C Termination Rate Class.

ToS Type of Service.

TRR Termination Rates Recommendation.

UB User Behavior. 
UE User Equipment.

UI User Interface.

VCG Vickrey-Clarke-Groves.

VLR Visitor Locator Register.

VoIP Voice over Internet Protocol.

W3 C World Wide Web Consortium.

WebRTC Web Real-Time Communications.

WLAN Wireless Local Area Network. 
